hep-th/0307154

July 2003

\title{
Renormalization group flows and continual Lie algebras
}

\author{
Ioannis Bakas \\ Department of Physics, University of Patras \\ GR-26500 Patras, Greece \\ bakas@ajax.physics. upatras.gr
}

\begin{abstract}
We study the renormalization group flows of two-dimensional metrics in sigma models using the one-loop beta functions, and demonstrate that they provide a continual analogue of the Toda field equations in conformally flat coordinates. In this algebraic setting, the logarithm of the world-sheet length scale, $t$, is interpreted as Dynkin parameter on the root system of a novel continual Lie algebra, denoted by $\mathcal{G}(d / d t ; 1)$, with anti-symmetric Cartan kernel $K\left(t, t^{\prime}\right)=\delta^{\prime}\left(t-t^{\prime}\right)$; as such, it coincides with the Cartan matrix of the superalgebra $\operatorname{sl}(N \mid N+1)$ in the large $N$ limit. The resulting Toda field equation is a non-linear generalization of the heat equation, which is integrable in target space and shares the same dissipative properties in time, $t$. We provide the general solution of the renormalization group flows in terms of free fields, via Bäcklund transformations, and present some simple examples that illustrate the validity of their formal power series expansion in terms of algebraic data. We study in detail the sausage model that arises as geometric deformation of the $O(3)$ sigma model, and give a new interpretation to its ultra-violet limit by gluing together two copies of Witten's two-dimensional black hole in the asymptotic region. We also provide some new solutions that describe the renormalization group flow of negatively curved spaces in different patches, which look like a cane in the infra-red region. Finally, we revisit the transition of a flat cone $C / Z_{n}$ to the plane, as another special solution, and note that tachyon condensation in closed string theory exhibits a hidden relation to the infinite dimensional algebra $\mathcal{G}(d / d t ; 1)$ in the regime of gravity. Its exponential growth holds the key for the construction of conserved currents and their systematic interpretation in string theory, but they still remain unknown.
\end{abstract}




\section{Introduction}

Vacuum selection in string theory is an important problem that remains unsolved to this day. Different vacua are constructed by imposing conformal invariance on the world-sheet quantum field theories, which yield classical solutions of the space-time field equations as fixed points of the two-dimensional renormalization group equations. Then, in this context, the transitions among vacua can be studied by considering the space of all two-dimensional quantum field theories and use the renormalization group flows to interpolate between different classical solutions. Non-conformal backgrounds provide a way to continue off-shell in string theory, in which case the world-sheet renormalization group equations hold the key for exploring the structure of the infinite dimensional space of all possible string configurations. One may also assume that there is an evolution in physical time, which can be identified with the world-sheet renormalization group time, but this on-shell formulation has not been made systematic in the general case. There are compelling reasons to expect that the transitions can be addressed equally well in these two different frames, since fixed points of the renormalization group flows are stationary solutions for classical strings and there is no known decay process for which there is no corresponding renormalization group flow. We will adopt the renormalization group approach in the gravity regime and present some new ideas for the reformulation of string dynamics in terms of a novel infinite dimensional Lie algebra with exponential growth. This framework will also prove useful for exploring the integrability properties of the renormalization group flows in target space.

The renormalization group flows lead to transitions from unstable extrema towards more stable ones by the condensation of tachyons in various string models. It is also important to realize in this context that a deeper understanding of non-supersymmetric string dynamics may help to connect string theory with the real world. The problem of tachyon condensation is fairly well understood in open string theory, following the seminal work by Sen [1, 2], Witten [3], and many others, but in closed string theory it is more difficult and remains largely unexplored. The main complication is the presence of gravity, which makes it difficult to isolate the condensation phenomena and their properties. Closed string tachyon condensation has been recently addressed in a number of special situations, where tachyons are localized at a defect, such as branes or an orbifold fixed point [4, 5, 6, 7, 8, 9, 10]. Some of these results will be revisited in the present work in order to compare our new algebraic framework with what is already known about them. From this point of view, the framework we are proposing here is only the beginning for the algebraic reformulation of vacuum selection in closed string theory, where gravity plays a prominent role. However, further work is definitely required in order to make progress on the general problem of tachyon condensation, using the hidden relation to infinite dimensional Lie algebras.

We will study geometric transitions induced by the renormalization group flows of two-dimensional sigma models in the gravity regime by limiting our attention to the simplest case of two-dimensional target spaces and taking into account only the one- 
loop contribution to the beta function equations. We will be able to systematize the action of the renormalization group flows, as well as the construction of their general solution, using an integrable Toda field equation. This equation arises for purely metric backgrounds in conformally flat coordinates and provides a non-linear generalization of the heat equation in target space. The algebraic reformulation is made possible using an infinite dimensional Lie algebra with generalized Cartan kernel $K\left(t, t^{\prime}\right)=\delta^{\prime}\left(t-t^{\prime}\right)$, which falls into the general class of the so-called continual Lie algebras [11, 12, 13]. The Cartan variable $t$ (or $t^{\prime}$ ) plays the role of the logarithmic world-sheet length scale that drives the renormalization group flows, whereas the flow equations admit a zero curvature formulation as integrable two-dimensional system in target space based on this infinite dimensional algebra.

Although infinite dimensional Lie algebras have been used on several occasions in physics and mathematics, the particular algebra that arises in the theory of renormalization group flows has been studied only very little in the mathematics literature. This bosonic algebra is rather special for two main reasons. First, the first order dependence of the beta function on the renormalization group time, $t$, implies that the algebra has an anti-symmetric Cartan kernel, which happens to coincide with the Cartan matrix of the Lie superalgebra $\operatorname{sl}(N \mid N+1)$ for a certain choice of the simple odd root system when $N \rightarrow \infty$. As we will see later, this large $N$ limit is naturally defined by taking a continuous analogue of the Dynkin diagram, which justifies the use of the term "continual" for the corresponding Lie algebra. Second, this infinite dimensional Lie algebra exhibits exponential growth, which can be seen by taking successive commutators of the basic system of its Cartan-Weyl generators. This special property makes it rather difficult but worth studying in the context of string theory. It is natural to expect that higher modes of the string can be accommodated in the exponential growth of the Lie algebra elements at higher levels, and they can be subsequently used to describe beta functions beyond the metric deformations that are only addressed in this paper.

The ability to apply this new algebraic framework to the problem of tachyon condensation, in all generality, appears to be intimately related to the knowledge of the complete structure of the algebra, which remains unsolved problem. After all, other classes of generalized Kac-Moody algebras, like hyperbolic algebras, are conjectured symmetries of string theory via the vertex operator construction of higher string states, but their complete algebraic structure is also still lacking in all generality (see, for instance, [14, 15, 16, 17] and references therein). At the same time, the exponential growth of the renormalization group algebra holds the key for the construction of conserved currents in target space, and their systematic interpretation in string theory. Finally, it could be used as a framework for the algebraic definition of an entropy function that determines the dynamics of string theory in renormalization group time, and for the selection of stable vacua in the space of all two-dimensional quantum field theories. The intuitive expectation that the energy of space-time decreases along the renormalization group flow when there are transitions to more stable vacua (see, for instance, [5]), as well as Zamolodchikov's $c$-theorem that provides a monotonic function on the trajectories as 
they run from the ultra-violet to the infra-red fixed points [18] (but see also [19], 20]), might also admit a systematic interpretation in the framework of this particular infinite dimensional Lie algebra.

Thus, it becomes clear that the algebraic framework we are proposing in this paper sets up the stage for future developments in the renormalization group approach to offshell string theory. Here, it is predominately used to prove the integrability of the metric beta function equations in target space, at least to lowest order in the perturbative renormalization of the world-sheet sigma models, and parametrize their general solution in terms of free fields via Bäcklund transformations. We examine several special solutions in this context by considering geometric deformations that depend on a finite number of moduli for compact, as well as non-compact two-dimensional target spaces. These examples are used further to deepen our understanding of the geometric and algebraic aspects of the renormalization group equations, and their interrelation. Solutions with conical curvature singularities are also considered in detail, since their resolution in the infra-red region of the renormalization group flow serves as prototype for studying the problem of tachyon condensation in orbifold models.

It is natural to expect that the behavior of the renormalization group flows in the vicinity of singular geometries, and other unstable vacua, will admit a systematic description in the context of Toda field equations. The resulting integrable equation provides a non-linear generalization of the heat equation, but there is no general proof that it exhibits the same dissipative properties by diffusing in space any initial singular data after infinite long time. At this stage, one may only use the heat equation to approximate the Toda field equation for all times by studying the transition of a two-dimensional cone $C / Z_{n}$ to $C / Z_{m}$ with $n<m$ when both $n$ and $m$ are very large [4]. In all other cases the approximation is valid only asymptotically in the infra-red region, which is very far away from any initial singular data, but there are also exact results that are indicative of the dissipative properties of the non-linear equation. In any case, we expect to be able to provide a general proof of the dissipative properties of the renormalization group equations elsewhere, using the algebraic structure of the corresponding Toda system. This is also bound to have profound applications to the algebraic description of tachyon condensation based on the properties of the underlying infinite dimensional Lie algebra.

Most of the applications we will consider in the present work are not directly related to string theory, but they are included as testing ground for the algebraic formulation of the renormalization group flows as Toda system. Geometric deformations of constant curvature spaces, which can be either positive or negative, do not interpolate between an ultra-violet and an infra-red fixed point, as it is usually required for addressing the problem of vacuum selection in string theory. Positive curvature spaces become asymptotically free in the ultra-violet region and they reach a state of big crunch at some finite time, where strong curvature singularities are present and the lowest order approximation to the beta function equations breaks down. Negative curvature spaces, on the other hand, may start from a state of infinite negative curvature and flow towards a free theory in the infra-red region, which is well defined within the lowest order approximation to 
their beta function equations. Thus, apart from the special solutions that describe the decay of a cone $C / Z_{n}$ to $C / Z_{m}$, it will be very interesting to know other explicit solutions that interpolate between different string vacua. Unfortunately, there are no general solutions with this property in our disposal, as we do not yet have a systematic algebraic prescription for selecting the relevant configurations of the continual Toda field equation.

A few clarifying remarks are also put in order to avoid other possible sources of confusion. It appears as if the integrable structure we are advocating for the renormalization group flows is in contradiction with the dissipative properties of the time evolution, which resolve singularities and lead to the decay of unstable vacua to more stable ones. Dissipation is certainly not a property of integrable systems, but in our case space and time are treated differently. The zero curvature formulation of the flows leads to a relativistic system of second order equations in two-dimensional target space, whereas the time dependence is first order and it is encoded in the defining relations of the continual Lie algebra used in the Lax pair. Thus, it is still possible to have integrability in target space and dissipation in time. Put it differently, if we were able to formulate the transition among any two vacua as a dynamical process in real time, and not just as a flow in renormalization group time, we would have devised an ansatz for time dependent string backgrounds using a certain frame with specific $2+1$ (or more generally $9+1$ ) decomposition of space and time. The selection of a preferred time direction breaks general reparametrization invariance in space-time and the resulting equations may look asymmetric in the space and time variables in this particular frame. What is more surprising, however, is the apparent ability to model dissipative phenomena and decay processes in terms of infinite dimensional algebras in a systematic way. We will develop this formalism for the case of renormalization group flows of two-dimensional sigma models, but we suspect that it may also have far reaching consequences to other physical problems of this kind, including the systematic investigation of time dependent backgrounds in general relativity and in string theory.

The best we can presently do in order to relate the renormalization group flows with space-time dynamics is to employ the light-front evolution. It is known that any nonconformal gravitational background in $d$ dimensions can be used to define a conformally invariant theory in $d+2$ space-time dimensions using the embedding [21]

$$
d s^{2}=-2 d u d v+G_{i j}(u, x) d x^{i} d x^{j}, \quad \Phi^{\prime}=-v+\Phi(u, x)
$$

and identify the null coordinate $u$ with the renormalization group time $t$, as $u=t$. This embedding, which has been written here in all generality for non-conformal backgrounds with metric and dilaton fields $(G, \Phi)$ implies that the renormalization group flow becomes the profile of a gravitational wave in $(d+2)$-dimensional space-time, and it can be applied to all solutions that will be encountered in subsequent sections. Here, we also see that the coordinates of transverse space and the light-cone coordinate $u$ are treated differently to account for the different order of the differential equations in space and time variables. We also note for completeness that other deformations of two-dimensional conformal field theories can be made dynamical by constructing time dependent solutions of string 
theory in $d+1$ space-time dimensions, which describe changes of topology and other interesting transitions (see, for instance, [22, 23]), but it is not known how to achieve this embedding for arbitrary deformations including the ones we are considering here (see, however, some recent results described in [24]).

It is also worth emphasizing that the integrability of the renormalization group flows in the space of all target metrics looks very different in nature from the integrable deformations of conformal field theories away from criticality that were studied extensively some years ago. In all cases, one may describe the breaking of conformal invariance by adding appropriate operators to the world-sheet action, which are schematically represented by perturbing the initial conformal background

$$
S_{\mathrm{t}}=S_{0}+g(t) \int d^{2} w \mathcal{O}(w, \bar{w}) .
$$

There are special operators $\mathcal{O}$ that lead to deformations of the two-dimensional conformal field theory and which are integrable with respect to the world-sheet coordinates, as those initially studied by Zamolodchikov [25]. There are other deformations that correspond to adding relevant operators on the world-sheet, which are tachyonic in space-time, and which describe tachyon condensation in string theory by flowing towards the infra-red region of the renormalization group flow. The latter are not integrable on the world-sheet, but they provide special solutions of an integrable system in target space within the context of our discussion. In fact, it will be interesting to know whether all different kind of perturbations away from criticality, apart from the metric perturbations we are considering in the present work, can be viewed as special solutions of a big integrable structure in target space that casts the beta function equations of all fields in zero curvature form. Thus, we may have integrability on the world-sheet only for some special choices of $\mathcal{O}$, whereas integrability in target space appears to be a property of the superspace of all two-dimensional quantum field theories. As noted before, the complete structure of the renormalization group algebra and its systematic interpretation in string theory hold the key to the general formulation of this proposal.

The remaining sections of this paper are organized as follows. In section 2 , we include some background material and formulate the renormalization group equations for metric backgrounds in different frames. The equations assume rather simple form for two-dimensional target spaces in conformally flat coordinates, where they can be formally viewed as Toda system. As such, they provide a non-linear generalization of the heat flow equation whose basic properties, as well as its fundamental solutions, are also reviewed. In section 3, we provide a systematic description of the renormalization group flows as integrable Toda system, using an infinite dimensional Lie algebra with exponential growth. This algebra is defined in the framework of continual Lie algebras by identifying the Dynkin parameter of its root system with the renormalization group time. Then, using the zero curvature formulation of the beta function equations, we present the general solution in terms of arbitrary families of free fields via Bäcklund transformations. The general solution is also expressed as formal power series expansion about the corresponding free field configurations by making use of the Lie algebra commutation relations 
and its formal highest weight representations, in close analogy with finite dimensional Toda systems. We also formulate some generalized Toda field equations, which could be relevant for the systematic description of other renormalization group equations beyond the metric deformations. In section 4, we revisit the sausage model which represents special deformations of the $O(3)$ sigma model under the renormalization group flows. We also describe the deformation in proper coordinates and give a new interpretation to the ultra-violet limit of model by gluing together two copies of Witten's two-dimensional black hole in the asymptotic region. This example proves particularly useful for illustrating the validity of the formal power series expansion of the general solution to Toda field equation in terms of free fields. In section 5, we extend the discussion to special geometric deformations of constant negative curvature metrics in two dimensions, using different patches that correspond to hyperbolic, parabolic or elliptic configurations. We find that the target space looks like a cane in the infra-red region, apart from the elliptic case which has a conical curvature singularity. In section 6 , we consider more complicated solutions of the continual Toda field equation that describe the decay of conical singularities under the renormalization group flows. The transition from the cone $C / Z_{n}$ to $C / Z_{m}$ with $m<n$, and eventually to a two-dimensional plane, serves as prototype for studying the behavior of the general solution close to curvature singularities. The end-point of the flow is reached by diffusing the curvature singularity all over the space and, thus, the existence of these special solutions indicates the dissipative nature of the non-linear evolution, as in the linearized heat equation. Applications to the problem of tachyon condensation are also briefly discussed in this context. Finally, in section 7, we present our conclusions and indicate some directions for future work.

\section{Renormalization group flows of sigma models}

The renormalization group properties of two-dimensional sigma models have attracted considerable attention following the seminal work by Polyakov on the $O(N)$ sigma model,

$$
S=\frac{1}{g^{2}} \int d^{2} w(\partial \vec{n})^{2}
$$

with $\vec{n}$ being an $N$-dimensional unit vector, $\vec{n} \cdot \vec{n}=1$. It was found that the theory is perturbatively renormalizable and the beta function $\beta\left(g^{2}\right)$ is negative for all nonabelian groups with $N \geq 3$, due to the positive curvature of the target space manifold $S^{N-1}=S O(N) / S O(N-1)$. More precisely, introducing a momentum cut-off $\Lambda$, the coupling $g^{2}$ changes as follows, [26, 27],

$$
\frac{1}{\tilde{g}^{2}}=\frac{1}{g^{2}}+\frac{N-2}{4 \pi} \log \frac{\tilde{\Lambda}}{\Lambda}
$$

and, therefore, $\beta\left(g^{2}\right)=-(N-2) g^{4} / 4 \pi$ to lowest order. Consequently, the theory becomes asymptotically free in the ultra-violet region, in close analogy with the more complicated 
example of non-abelian gauge theories in four dimensions. For $N=2$, the theory is conformal and describes the physics of a single compact scalar field in two dimensions.

Further generalizations were also considered later by studying the renormalization group equations for arbitrary background metrics in target space with far reaching consequences to the development of string theory (see, for instance, 28, 29] and references therein). Here, we consider the structure of the renormalization group equations away from fixed points and present some new ideas that set up the basis for their integrability in target space for the simplest case of two-dimensional manifolds.

\subsection{General considerations}

Generalized two-dimensional sigma models are well studied both classically and quantum mechanically. Classically, they are described by the action

$$
S=\frac{1}{2} \int d^{2} w \sqrt{h} h^{i j}\left(\partial_{i} X^{\mu}\right)\left(\partial_{j} X^{\nu}\right) G_{\mu \nu}
$$

where $\left\{w^{i} ; i=1,2\right\}$ are coordinates on the world-sheet (base space) with metric $h_{i j}(w)$ and $\left\{X^{\mu} ; \mu=1,2, \cdots, n\right\}$ are coordinates in target space with metric $G_{\mu \nu}(X)$. The standard approach to quantization is via perturbation theory. When the curvature of the target space geometry is small, the sigma model action is perturbatively renormalizable and it can be treated by adding counter terms of the form

$$
S_{\text {c.t. }}=\frac{1}{2} \int d^{2} w \sqrt{h} h^{i j}\left(\partial_{i} X^{\mu}\right)\left(\partial_{j} X^{\nu}\right) \mathcal{R}_{\mu \nu},
$$

which cancel the ultra-violet divergencies of the theory. $\mathcal{R}_{\mu \nu}$ is a symmetric tensor that depends on the curvature and it can be computed systematically at one-loop or higher orders [30, 31] (but see also [32, 33, 34] among many other relevant references).

The renormalization group equations of two-dimensional sigma models are

$$
\Lambda^{-1} \frac{\partial}{\partial \Lambda^{-1}} G_{\mu \nu} \equiv-\beta\left(G_{\mu \nu}\right)=-R_{\mu \nu}-\frac{1}{2} R_{\mu \rho \sigma \tau} R_{\nu}{ }^{\rho \sigma \tau}+\cdots
$$

where $R_{\mu \nu}$ is the Ricci curvature tensor of the target space metric that provides the oneloop contribution to $\mathcal{R}_{\mu \nu}$ in appropriate units, $2 \pi \alpha^{\prime}=1$. The quadratic terms arise at two loops and they involve contributions from the Riemann curvature tensor, whereas higher order terms, which are denoted by dots, account for higher order curvature terms at higher loops. $\Lambda^{-1}$ sets the world-sheet renormalization scale parameter, which can be traded with the logarithmic length scale defined by $t=\log \Lambda^{-1}$. Using this variable, which is usually called the renormalization group time, the ultra-violet limit is reached as $t \rightarrow-\infty$, whereas the infra-red limit lies at $t \rightarrow+\infty$. Then, to lowest order in perturbation theory, we have the equations

$$
\frac{\partial}{\partial t} G_{\mu \nu}=-R_{\mu \nu}
$$


which describe changes of the target space geometry induced by changes of the worldsheet logarithmic length scale.

Their integration yields trajectories in superspace, which consists of all Riemannian metrics ${ }^{1}$. The problem is quite complicated in all generality, as superspace is an infinite dimensional manifold and the renormalization group equations act as dynamical system in the time variable, $t$. We do not know how to treat this system of equations for all target space manifolds, but many simplifications occur when the dimension of target space is two and the equations become more tractable. We will in fact show that the system becomes integrable in target space when $n=2$, using a novel infinite dimensional Lie algebra which is introduced later to rewrite the renormalization group equations in zero curvature form. This is made possible to lowest order in perturbation theory by omitting all higher order curvature terms. Inclusion of higher order terms, which apparently become important when the geometry develops strong curvature, may change our conclusions but their effect will not be addressed in the present work. We are also excluding the effect of other fields which might be present in the given class of string backgrounds, and leave their treatment to future work.

There are a few general results one can derive for the action of the renormalization group flows on geometric collective parameters of two-dimensional sigma models. We first note that the volume $V$ of the target space manifold $M$,

$$
V=\int_{M} \sqrt{\operatorname{det} G} d^{n} X
$$

changes under the renormalization group flow as follows,

$$
\frac{\partial V}{\partial t}=\frac{1}{2} \int_{M} d^{n} X \sqrt{\operatorname{det} G} G^{\mu \nu} \frac{\partial G_{\mu \nu}}{\partial t}=-\frac{1}{2} \int_{M} d^{n} X \sqrt{\operatorname{det} G} R[G],
$$

using the identity $2 \partial(\sqrt{\operatorname{det} G}) / \partial t=\sqrt{\operatorname{det} G} G^{\mu \nu} \partial G_{\mu \nu} / \partial t$. The rate of change is given by the Einstein-Hilbert action of the target space metric and the result is certainly valid in any number of dimensions. In two dimensions, in particular, $n=2$, which is our main concern, the Einstein-Hilbert action is topological and it provides the Euler number of the target space manifold, namely

$$
\chi(M)=\frac{1}{4 \pi} \int_{M} d^{2} X \sqrt{\operatorname{det} G} R[G] .
$$

Therefore, we deduce the linear dependence of the two-dimensional target space volume

$$
V(t)=V_{0}-2 \pi t \chi(M)
$$

where $V_{0}$ is an arbitrary integration constant equal to the volume of the space at $t=0$.

For compact spaces with positive Euler number, $V_{0}$ is finite and it can be normalized to $V_{0}=2 \pi t_{0} \chi(M)$, by introducing a positive definite time scale $t_{0}$. Then, we may write

$$
V(t)=2 \pi\left(t_{0}-t\right) \chi(M)
$$

\footnotetext{
${ }^{1}$ It should not be confused with the notion of superspace used in the formulation of supersymmetric field theories.
} 
where $t_{0}$ corresponds to the renormalization group scale for which $V\left(t_{0}\right)=0$ (see also 35]). The structure of the renormalization group equations predicts in this case that the manifold shrinks to zero size at some given instant of time, $t=t_{0}$. Strong curvature singularities will develop close to the big crunch, which may invalidate the lowest order description of the renormalization group equations. In view of this behavior, it only makes sense to consider flows from the ultra-violet region to $t_{0}$. The $O(3)$ sigma model falls precisely in this class, as it has positive curvature and $\chi\left(S^{2}\right)=2$, and exhibits ultra-violet asymptotic freedom. On the other hand, when the space is compact with Euler number $\chi(M)<0$, as in the case of all Riemann surfaces with genus $g \geq 2$, which also have negative curvature, it only makes sense to consider the action of the renormalization group flows from some finite time to the infra-red region. For the torus, $S^{1} \times S^{1}$, which is a Riemann surface with genus 1 and Euler number 0 , the volume remains unchanged, as the two-dimensional field theory is conformal and the metric does not run. Finally, $V_{0}$ is infinite for non-compact manifolds, in which case the renormalization group flows may end up at infra-red geometries with variable volume, depending on the parameters of specific trajectories. This behavior will be encountered in section 5 by considering geometric deformations of constant negative curvature spaces in different patches. Strictly speaking, however, it is appropriate to regularize the volume of noncompact target spaces before considering the evolution under the renormalization group flow.

It is also natural to inquire at this point whether the topology of the target space $M$ can change under the renormalization group flows, given the drastic geometric deformations that $M$ undergoes in time. This question can be examined in all target space dimensions, but in two dimensions, in particular, we may only consider the flow of the Euler number. It turns out that $\partial \chi(M) / \partial t=0$, because in two dimensions we have identically $2 R_{\mu \nu}=G_{\mu \nu} R$. Therefore, we conclude that the Euler number of the target space manifold remains invariant under the flow, as expected.

The results we have obtained so far show that there exist topological as well as geometrical integrals of the renormalization group flow in two dimensions, which are given by the quantities $I_{1}=\chi(M)$ and $I_{2}=V+2 \pi t I_{1}$ and satisfy conservation laws in time, $\partial I_{i} / \partial t=0$. Actually, these might be simple examples from an extended list of higher integrals of motion, which are currently unknown. Although this problem will not be investigated in the present work, we note that there is a way to proceed systematically by embedding the two-dimensional manifold $M$ in three-dimensional Euclidean space. Such an embedding may also be used in order to provide a concrete visualization of the geometric deformations of $M$ under the renormalization group flows. Since any embedding problem requires knowledge of the metric $G_{\mu \nu}$, as well as the extrinsic curvature tensor $K_{\mu \nu}$, it is natural to expect that higher integrals of motion, if they exist at all in the time variable $t$, will involve components of the extrinsic curvature tensor. Integrals of the extrinsic curvature tensor may also be used to characterize the rigidity of the space and the response of its shape under the renormalization group flow via the associated system of Gauss-Codazzi equations. This provides a well-posed problem in differential 
geometry, which has not been investigated so far for the special geometric deformations (2.6), to the best of our knowledge.

\subsection{Renormalization group equations in different frames}

The renormalization group equations (2.6) generalize when arbitrary reparametrizations of the target space coordinates take place along the flows. The reparametrizations are elements of the diffeomorphism group of the manifold $M$ and they are generated by vector fields $\xi_{\mu}$ that may depend on all target space coordinates, as well as on time. Then, the beta function equations of a purely metric background assume the generalized form [31]

$$
-\beta\left(G_{\mu \nu}\right) \equiv \frac{\partial}{\partial t} G_{\mu \nu}=-R_{\mu \nu}+\nabla_{\mu} \xi_{\nu}+\nabla_{\nu} \xi_{\mu}
$$

They incorporate the effect of all possible field redefinitions $\delta X_{\mu}=-\xi_{\mu}$, which is a poor man's way to describe diffeomorphisms, but they can be excluded if we are only interested in changes of the geometry itself (see also 32, 34]). Also, no additional terms arise for reparametrizations associated to Killing vector fields, as they satisfy $\nabla_{\mu} \xi_{\nu}+\nabla_{\nu} \xi_{\mu}=0$ by definition.

In order to further understand the meaning of such additional terms, it is useful to write down the renormalization group equations for both metric and dilaton fields, $G_{\mu \nu}$ and $\tilde{\Phi}$, which assume the following form in the sigma model frame:

$$
\begin{aligned}
& \frac{\partial}{\partial t} G_{\mu \nu}=-R_{\mu \nu}-2 \nabla_{\mu} \nabla_{\nu} \tilde{\Phi}+\nabla_{\mu} \xi_{\nu}+\nabla_{\nu} \xi_{\mu}, \\
& \frac{\partial}{\partial t} \tilde{\Phi}=-(\nabla \tilde{\Phi})^{2}+\frac{1}{2} \nabla^{2} \tilde{\Phi}+\xi_{\mu} \nabla^{\mu} \tilde{\Phi} .
\end{aligned}
$$

Then, it is always possible to choose a frame by appropriate reparametrizations generated by the vector field $\xi_{\mu}=\partial_{\mu} \tilde{\Phi}$, so that the system of the renormalization group equations simplifies to

$$
\frac{\partial}{\partial t} G_{\mu \nu}=-R_{\mu \nu}, \quad \frac{\partial}{\partial t} \tilde{\Phi}=\frac{1}{2} \nabla^{2} \tilde{\Phi},
$$

and provides a simple extension of the metric beta function equation (2.6) $)$. According to this simple observation, the effect of the dilaton is similar to the effect of an arbitrary reparametrization generated by the vector field $\xi_{\mu}$.

We also recall at this point that the one-loop effective action of two-dimensional sigma models exhibits conformal invariance only on-shell under the naive conformal transformation laws

$$
\delta_{\epsilon} h_{i j}=\epsilon h_{i j}, \quad \delta_{\epsilon} X^{\mu}=0 .
$$

Conformal invariance can be achieved off-shell by writing down the modified Weyl transformation laws

$$
\delta_{\epsilon} h_{i j}=\epsilon h_{i j}, \quad \delta_{\epsilon} X^{\mu}=\epsilon G^{\mu \nu} \partial_{\nu} \tilde{\Phi}
$$


which are applicable to both cases: $\tilde{\Phi}$ can be either the dilaton or the "would be" dilaton that acts as potential for the vector field $\xi_{\mu}$. Then, the meaning of the simplified system of equations (2.14) is that conformal invariance can be reinstated under the transformation (2.15) by arranging the reparametrizations to cancel the effect of the dilaton on the modified Weyl transformations (2.16). Thus, reparametrizations assign non-trivial Weyl transformation laws to the target space coordinates and act as non-trivial dilaton gradient in the renormalization group flows of two-dimensional metric sigma models. We will make extensive use of this well known fact in subsequent sections by changing frames from conformal to proper coordinates.

Let us now restrict attention to two-dimensional target spaces in the conformally flat frame

$$
d s_{\mathrm{t}}^{2}=2 e^{\Phi\left(z_{+}, z_{-} ; t\right)} d z_{+} d z_{-}=\frac{1}{2} e^{\Phi(X, Y ; t)}\left(d X^{2}+d Y^{2}\right)
$$

using the Cartesian coordinates $X, Y$ or the complex conjugate variables $2 z_{ \pm}=Y \pm i X$. It is well-known that any two-dimensional metric can always be written in this form, at least locally, for appropriately chosen conformal factor $\Phi$, in which case the non-vanishing components of the Ricci curvature tensor are

$$
R_{+-}=-\partial_{+}\left(e^{-\Phi} \partial_{-} e^{\Phi}\right) \equiv-\nabla^{2} \Phi
$$

Here, for notational purposes, we write $\nabla^{2}=\partial^{2} / \partial z_{+} \partial z_{-}=\partial^{2} / \partial X^{2}+\partial^{2} / \partial Y^{2}$. Then, the renormalization group equations (2.6) simplify to the following non-linear differential equation for $\Phi(X, Y ; t)$,

$$
\frac{\partial}{\partial t} e^{\Phi(X, Y ; t)}=\nabla^{2} \Phi(X, Y ; t)
$$

which has to be solved for all $t$.

It proves advantageous for the purposes of the present work to rewrite this equation in the form

$$
\nabla^{2} \Phi(X, Y ; t)=\int d t^{\prime} K\left(t, t^{\prime}\right) e^{\Phi\left(X, Y ; t^{\prime}\right)}
$$

where $K\left(t, t^{\prime}\right)$ is the kernel

$$
K\left(t, t^{\prime}\right)=\frac{\partial}{\partial t} \delta\left(t-t^{\prime}\right)
$$

This equation admits a natural algebraic interpretation in the framework of Toda field theory, which is an integrable system of non-linear differential equations in two dimensions with local coordinates $\left(z_{+}, z_{-}\right)$. In Toda theory we consider a collection of non-linear fields $\left\{\phi_{i}\left(z_{+}, z_{-}\right)\right\}$, which are are labeled by the simple roots of a given Lie algebra and interact via the Cartan matrix $K_{i j}$ of the Lie algebra, as follows,

$$
\nabla^{2} \phi_{i}(X, Y)=\sum_{j} K_{i j} e^{\phi_{j}(X, Y)}
$$

The indices $i$ and $j$ are typically discrete, but there are also generalizations to continuous variables for some infinite dimensional Lie algebras, which are obtained by replacing the collection of two-dimensional Toda fields $\left\{\phi_{i}\right\}$ with a "master" field $\Phi\left(z_{+}, z_{-} ; t\right)$, the Cartan matrix $K_{i j}$ with a Cartan kernel $K\left(t, t^{\prime}\right)$, and the summation over $j$ by an integral 
over $t^{\prime}$. Then, in this framework, the renormalization group equation (2.19) can be viewed as limiting case of a Toda system with Cartan matrix

$$
K_{i j}=\delta_{i+1, j}-\delta_{i, j+1}
$$

when the discrete indices are replaced by the continuous variable $t$. This formal description of the non-linear equation (2.19) is actually very precise in the context of infinite dimensional continual Lie algebras. The details will be given in section 3 together with all necessary background material.

The general solution of the renormalization group equation (2.19) can be written, at least formally, by appealing to the Toda field theory interpretation. Here, as in all integrable Toda theories, non-linear field configurations can be constructed group theoretically, via Bäcklund transformations, using arbitrary families of two-dimensional free fields. This algebraic method is outlined in section 3 in all generality and allows to integrate the evolution of two-dimensional sigma models in superspace using free field coordinates. However, it is convenient for practical purposes to develop a minisuperspace approach to the renormalization group equations by considering geometric deformations of the target space metric that depend only on a finite number of degrees of freedom, say $\left\{a_{i}(t)\right\}$. For consistent mini-superspace reductions of the field equation (2.19), the moduli $a_{i}(t)$ satisfy a system of first order differential equations that describe specific geometric deformations of the target space geometry, which are much easier to understand algebraically as well as geometrically. All explicit solutions that are worked out in subsequent sections are precisely of this form, and they can be used to check the validity of the general solution in terms of free fields.

There are situations, however, where the mini-superspace approximation is mathematically consistent, but the resulting solutions of the renormalization group equation are not physically sensible. Moreover, there can be instabilities in some mini-superspace models, in that the trajectories become very sensitive to relevant geometric deformations which were ignored at first sight. Although it is not easy to analyze these issues in all generality at this moment, using a stability analysis in the space of all possible metrics, we expect that the behavior of some rather simple geometries with conical singularities will help to understand the problem in general terms. Besides, the fate of singularities is also an issue of paramount importance in applications of the renormalization group flows to the problem of tachyon condensation in closed string theory. Examples of all different types will also be given later.

\subsection{The heat equation}

The renormalization group equation (2.19) for conformally flat two-dimensional metrics provides a non-linear generalization of the well-known heat equation in flat space,

$$
\frac{\partial}{\partial t} \Theta(X, Y ; t)=\nabla^{2} \Theta(X, Y ; t)
$$


Indeed, the renormalization group equation can be approximated by the heat equation when $\Phi(X, Y ; t)$ becomes very small, i.e., when the target space metric becomes approximately flat and smooth,

$$
G_{\mu \nu} \simeq \delta_{\mu \nu}, \quad \text { with } h_{\mu \nu}<<1 \text { and } \partial h_{\mu \nu}<<1
$$

This is typically valid in the infra-red region of the renormalization group flow, where $\Theta(X, Y ; t)$ also becomes very small and smooth, and we may set

$$
\Phi(X, Y ; t) \simeq \Theta(X, Y ; t), \quad \text { as } t \rightarrow+\infty
$$

Thus, under appropriate conditions, the heat equation provides a linearization of the renormalization group equation in the infra-red limit, where small fluctuations around the corresponding conformal field theory are well defined in the weak field approximation. We will present examples of this behavior in subsequent sections by considering theories with positive definite beta functions, which exhibit trivial infra-red fixed points.

The fundamental solution of the heat equation in one spatial dimension is a Gaussian pulse with height $1 / \sqrt{t}$ and width $\sqrt{t}$, whereas in two dimensions it is given explicitly by

$$
\Theta(X, Y ; t)=\frac{1}{4 \pi t} e^{-\left(X^{2}+Y^{2}\right) / 4 t}
$$

up to normalization. It starts as singular function at the initial time $t=0$, where the Gaussian function becomes delta function, and it spreads in time with diminishing height as $t \rightarrow+\infty$. As such, it encodes the dissipative properties of the heat equation that resolves in time the initial singularity. This elementary, but rather fundamental, decay process is presented schematically in figure 1 below.
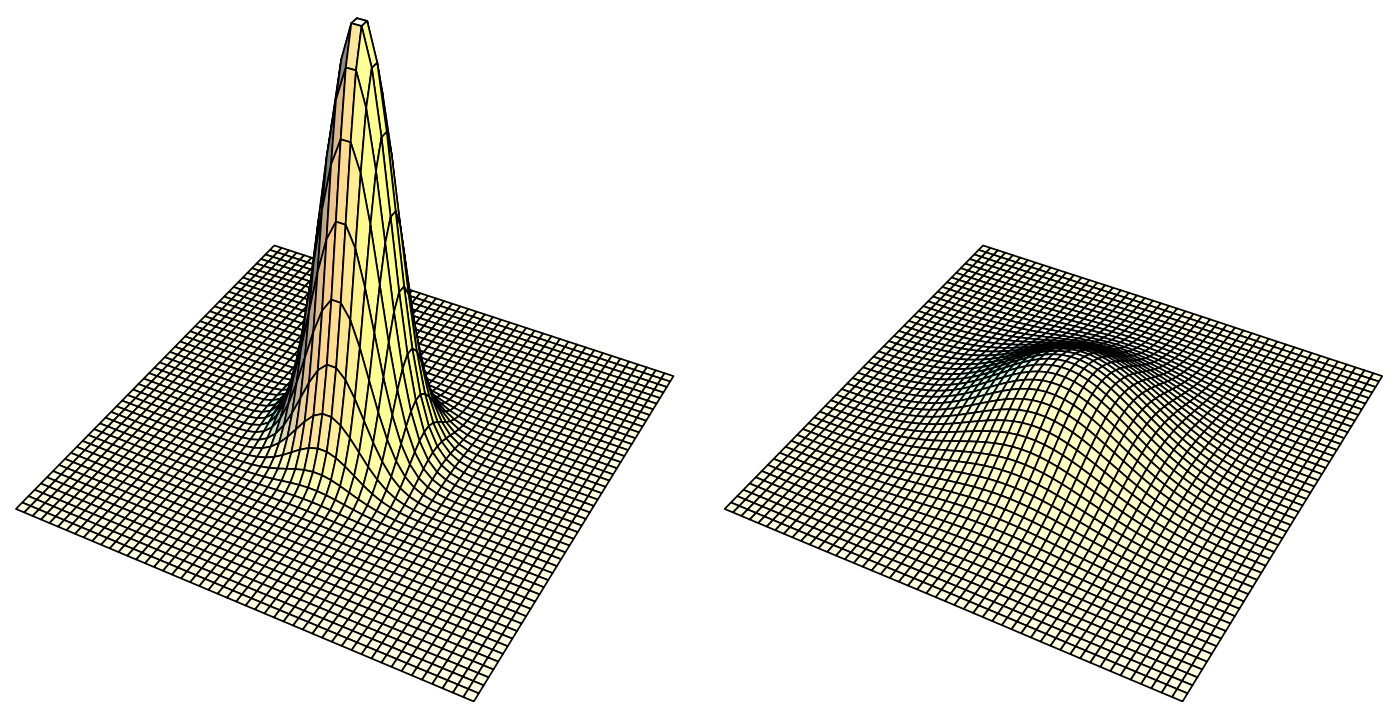

Figure 1: A Gaussian distribution spreading in time.

Since the weak field approximation is not valid for finite values of $t$, one may wonder whether the most characteristic property of the heat equation to dissipate singularities 
is also a property in the non-linear Toda field equation. Quite remarkably, the answer is affirmative with many important consequences to the problem of tachyon condensation in closed string theory. The most elementary example, which is the analogue of the fundamental solution to the heat equation, is provided by the two-dimensional cone $C / Z_{n}$. This geometry is everywhere flat apart from a point, the tip of the cone, where the curvature has delta function singularity. There is a special exact solution of the renormalization group equations in this case, which is valid for all $t$ and describes the decay of the initial conical singularity into nothing by spreading smoothly its curvature in space [4, 5]. The end-point of this process is a two-dimensional plane that arises as infra-red fixed point, while the curvature singularity is washed away in close analogy with the evolution of the Gaussian distribution depicted in figure 1. This characteristic solution of the continual Toda field equations will be examined closely in section 6 by expanding on earlier work by other authors.

More complicated solutions of the heat equation can be easily obtained by taking appropriate superposition of elementary Gaussian functions located at different points in space, thanks to its linearity. In one dimension, for example, one may consider the superposition of infinite many fundamental solutions of the heat equation on the line, $1 / \sqrt{4 \pi t} \exp \left(-z^{2} / 4 t\right)$, with initial data being a sum of delta functions for all integer values $z=n$. The function that results in this case is

$$
\Theta(z ; t)=\frac{1}{\sqrt{4 \pi t}} \sum_{n \in Z} \exp \left(-\frac{(z-n)^{2}}{4 t}\right)
$$

and provides a periodic solution of the heat equation with $\Theta(z+1 ; t)=\Theta(z ; t)$. Actually, this particular construction defines the Jacobi theta function, as it can be seen using the remarkable identity [36, 37.

$$
\Theta(z ; t)=\sum_{n \in Z} \exp \left(-4 \pi^{2} n^{2} t+2 \pi i n z\right)=\frac{1}{\sqrt{4 \pi t}} \sum_{n \in Z} \exp \left(-\frac{(z-n)^{2}}{4 t}\right) .
$$

The Jacobi theta function $\Theta(u ; \tau)$ follows from above by setting $u=2 z K(k)$ and $\tau=4 \pi i t$. All other theta functions, namely $H(u ; \tau), \Theta_{1}(u ; \tau)=\Theta(u+K(k) ; \tau)$ and $H_{1}(u ; \tau)=$ $H(u+K(k)) ; \tau)$ also satisfy the same heat equation ${ }^{2}$.

Parametrizing the complex modulus of the theta functions as $\tau=i K\left(k^{\prime}\right) / K(k)$, where $K(k)$ denotes the complete elliptic integral of the first kind with modulus $0 \leq k \leq 1$ and $k^{\prime}=\sqrt{1-k^{2}}$, we see immediately that $\Theta(Y ; t)$ describes a train of delta functions at $t=0$, or equivalently at $k=1$, which corresponds to the degeneration limit of the underlying Riemann surface when the $b$-cycle vanishes. On the other hand, the value $k=0$ is attained in the infra-red limit $t \rightarrow+\infty$, which corresponds to the degeneration limit of the underlying Riemann surface when the $a$-cycle vanishes. In this limit we have $\Theta(Y ; 0)=1$ and, likewise, the other theta functions yield $\Theta_{1}(Y ; 0)=0, H(Y ; 0)=0$

\footnotetext{
${ }^{2}$ In more standard notation, $H, H_{1}, \Theta_{1}$ and $\Theta$ correspond to the theta functions $\theta_{1}, \theta_{2}, \theta_{3}$ and $\theta_{4}$ respectively. This sets up the notation that will be used in subsequent sections. For a detailed account of their properties, we refer the reader to standard texts on elliptic functions (see, for instance, [36]).
} 
and $H_{1}(Y ; 0)=1$. Thus, all theta functions share the same dissipative property with the simple Gaussian function on the real line. An important feature of these periodic solutions is the interpretation of the complex modulus $\tau$ as Wick rotation of the real time variable $t$. Then, the time evolution is solely described by changes of the complex structure of the underlying Riemann surface between the two different degeneration limits.

We do not know any elliptic solutions of the renormalization group equation in conformal coordinates, but as we will see later, there are simple algebraic solutions of equation (2.19) which are expressed in terms of theta functions in another frame by employing proper coordinates. Then, the heat equation for the theta functions can be used to prove that these configurations satisfy the renormalization group equations for all times, and not only asymptotically; $t$ is also assigned to the shape modulus of a Riemann surface with $k=0$ corresponding to the infra-red limit. Put it differently, there are special ansatz for embedding solution of the heat equation into the generalized system of renormalization group equations (2.12), which are valid at all times. A more systematic understanding of these ansatz is definitely required in order to construct other interesting solutions in the future. This embedding might also be closely related to the linearization of the renormalization group equations, which is proved in the next section, and could be used further to explain why both equations share the same dissipative properties that lead to the resolution of initial singularities.

\section{Toda systems and Lie algebras}

In this section we review the basic properties of Toda field theories and outline the systematic description of their solutions in terms of two-dimensional free field configurations. We start with some preliminary material on Toda theories associated with finite dimensional Lie algebras with Cartan matrix $K_{i j}$, and then extend the discussion to infinite dimensional algebras with generalized Cartan matrix given by a kernel $K\left(t, t^{\prime}\right)$ that depends on a continuous parameter rather than a discrete Dynkin index $i$. These are the so called continual Lie algebras, which provide a general class of infinite dimensional Lie algebras that include, among other cases, the large $N$ limit of the finite dimensional simple Lie algebras $\operatorname{sl}(N)$. For the purposes of the present work, we focus on the case of a rather odd continual Lie algebra with anti-symmetric Cartan kernel $K\left(t, t^{\prime}\right)=\delta^{\prime}\left(t-t^{\prime}\right)$, which provides the renormalization group flow for the conformal factor of a two-dimensional metric as its Toda theory. In this new algebraic setting, the logarithm of the world-sheet length scale associated with the renormalization group time is interpreted as a continuous Dynkin parameter on the root system of this infinite dimensional Lie algebra; at the same time, its Cartan matrix happens to be identical to the continuous large $N$ limit of the superalgebra $\operatorname{sl}(N \mid N+1)$ for a certain choice of its odd root system. We will present the general solution of the associated Toda field theory as a formal series in terms of twodimensional free fields and also discuss some characteristic algebraic properties of this particular continual Lie algebra that prove its exponential growth. This algebraic frame- 
work will be applied in subsequent sections to study a variety of geometric transitions under the renormalization group flow.

\subsection{Toda equations for simple Lie algebras}

Recall first the essential facts about Toda field theories associated with a simple Lie algebra $\mathcal{G}$ of finite rank $n$. Let $\phi_{i}\left(z_{+}, z_{-}\right)$be a collection of two-dimensional fields with $i=1,2, \cdots, n$ and let $K_{i j}$ be the Cartan matrix of $\mathcal{G}$, which serves as the coupling matrix among them. Toda field theory is described by the following relativistic system of non-linear differential equations with exponential interactions,

$$
\partial_{+} \partial_{-} \phi_{i}\left(z_{+}, z_{-}\right)=\lambda^{2} \sum_{j=1}^{n} K_{j i} e^{\phi_{j}\left(z_{+}, z_{-}\right)}
$$

which turns out to be integrable (see, for instance, 38, 39, 40, 41]).

The integrability properties of the theory follow easily from the zero curvature formulation of these equations,

$$
\left[\partial_{+}+A_{+}\left(z_{+}, z_{-}\right), \partial_{-}+A_{-}\left(z_{+}, z_{-}\right)\right]=0
$$

where $A_{ \pm}\left(z_{+}, z_{-}\right)$are gauge connections with values in $\mathcal{G}$,

$$
A_{+}=\sum_{i=1}^{n}\left(\left(\sum_{j=1}^{n} K_{i j}^{-1} \partial_{+} \phi_{j}\right) H_{i}+\lambda X_{i}^{+}\right), \quad A_{-}=\lambda \sum_{i=1}^{n} e^{\phi_{i}} X_{i}^{-} .
$$

The elements $H_{i}, X_{i}^{ \pm}$with $i=1,2, \cdots, n$ form the system of Weyl generators of $\mathcal{G}$ and they satisfy the defining relations

$$
\left[H_{i}, X_{j}^{ \pm}\right]= \pm K_{i j} X_{j}^{ \pm}, \quad\left[X_{i}^{+}, X_{j}^{-}\right]=\delta_{i j} H_{j}, \quad\left[H_{i}, H_{j}\right]=0
$$

The "coupling constant" $\lambda^{2}$ that appears in the classical equations of motion can be set equal to 1 (or -1 ) by rescaling the coordinates $z_{ \pm}$, or shifting all $\phi_{i}$ by $2 \log \lambda$, provided that $\lambda \neq 0$. For $\lambda=0$, however, the Toda field equations become linear, as it can be readily seen. We will keep $\lambda$ as book keeping parameter for future reference, in order to gain better insight into the free field realization of the general solution, but later we may set $\lambda^{2}= \pm 1$, depending on the sign of the coupling, without loss of generality.

The complete structure of the algebra $\mathcal{G}$ is not described by the system of Weyl generators alone, but they are sufficient to generate the remaining elements. In particular, all other generators of $\mathcal{G}$ can be obtained by taking successive commutators of these basic elements, namely $\left[\cdots\left[\left[X_{i_{1}}^{+}, X_{i_{2}}^{+}\right], X_{i_{3}}^{+}\right], \cdots, X_{i_{m}}^{+}\right]$, and likewise for the $X_{i}^{-}$'s. Put it differently, $H_{i}$ and $X_{i}^{ \pm}$span the subspaces of the Lie algebra $\mathcal{G}_{0}$ and $\mathcal{G}_{ \pm 1}$ respectively, which form the so called local part of the Lie algebra, $\mathcal{G}_{-1} \oplus \mathcal{G}_{0} \oplus \mathcal{G}_{+1}$, whereas the other elements belong in the subspaces $\mathcal{G}_{ \pm 2}=\left[\mathcal{G}_{ \pm 1}, \mathcal{G}_{ \pm 1}\right], \mathcal{G}_{ \pm 3}=\left[\mathcal{G}_{ \pm 1}, \mathcal{G}_{ \pm 2}\right]$, and so on. Of course, we also have the Serre conditions, which are imposed on the various successive 
commutators and they effectively provide the number of all independent generators of the simple Lie algebra $\mathcal{G}$. The system of Weyl generators is not only important for establishing the integrability properties of the underlying Toda field equations, but it may also be used for describing their general solution. For this, we also need to introduce representations of the Lie algebra $\mathcal{G}$ based on a highest weight state $\mid j>$, so that

$$
X_{i}^{+}|j>=0, \quad<j| X_{i}^{-}=0, \quad H_{i}\left|j>=\delta_{i j}\right| j>
$$

with the normalization $<j \mid j>=1$.

It is well known that the Toda field equations admit Bäcklund transformations that relate its general configuration to two-dimensional free fields. A particularly simple way to describe the free field realization of $\phi_{i}\left(z_{+}, z_{-}\right)$requires the introduction of two matrices $M_{ \pm}\left(z_{ \pm}\right)$, which satisfy the first order equations

$$
\partial_{ \pm} M_{ \pm}\left(z_{ \pm}\right)=M_{ \pm}\left(z_{ \pm}\right)\left(\lambda \sum_{i=1}^{n} e^{f_{i}^{ \pm}\left(z_{ \pm}\right)} X_{i}^{ \pm}\right)
$$

Here, $f_{i}^{ \pm}\left(z_{ \pm}\right)$are $n$ arbitrary holomorphic and anti-holomorphic functions so that the fields

$$
\phi_{i}^{(0)}\left(z_{+}, z_{-}\right)=f_{i}^{+}\left(z_{+}\right)+f_{i}^{-}\left(z_{-}\right)
$$

satisfy the two-dimensional free field equation, $\partial_{+} \partial_{-} \phi_{i}^{(0)}=0$, for all values of $i$. The matrices $M_{ \pm}\left(z_{ \pm}\right)$can be obtained by simple integration, using the path-ordered exponentials

$$
M_{ \pm}\left(z_{ \pm}\right)=\mathcal{P} \exp \left(\lambda \int^{z_{ \pm}} d z_{ \pm}^{\prime} \sum_{i=1}^{n} e^{f_{i}^{ \pm}\left(z_{ \pm}^{\prime}\right)} X_{i}^{ \pm}\right)
$$

where, as usual, we have the ordering prescription

$$
\mathcal{P} \exp \left(\int^{z} d z^{\prime} A\left(z^{\prime}\right)\right)=\sum_{m=0}^{\infty} \int^{z} d z_{1} \int^{z_{1}} d z_{2} \cdots \int^{z_{m-1}} d z_{m} A\left(z_{m}\right) \cdots A\left(z_{2}\right) A\left(z_{1}\right)
$$

with $z_{m} \leq \cdots \leq z_{2} \leq z_{1} \leq z$. Here, the lower value in the integration range is left arbitrary to account for the integration constants in $M_{ \pm}$that arise by solving the corresponding first order equations in terms of path-ordered exponentials. Also, since $\partial M=M A$ implies $\partial M^{-1}=-A M$ for all $M$ and $A$, the inverse of (3.9) is defined by taking the opposite ordering of paths and changing $A$ to $-A$.

Then, according to the literature, the general solution of the Toda field equations is given by the formula [39, 40, 41]

$$
\phi_{i}\left(z_{+}, z_{-}\right)=\phi_{i}^{(0)}\left(z_{+}, z_{-}\right)-\sum_{j=1}^{n} K_{j i} \log <j\left|M_{+}^{-1}\left(z_{+}\right) M_{-}\left(z_{-}\right)\right| j>
$$

which thus provides the required free field realization of the non-linear Toda field theory configurations in closed form.

The parametrization of the general solution in terms of free fields $\phi_{i}^{(0)}\left(z_{+}, z_{-}\right)$allows to express the non-linear Toda fields $\phi_{i}\left(z_{+}, z_{-}\right)$as a power series expansion, using the 
book keeping parameter $\lambda$. The individual terms can be computed by first expanding the path-ordered exponentials $M_{ \pm}$in power series in $\lambda$ and then expressing the expectation values $<j\left|M_{+}^{-1}\left(z_{+}\right) M_{-}\left(z_{-}\right)\right| j>$ as superposition of the Lie algebra matrix elements

$$
D_{j}^{\left\{i_{1}, i_{2}, \cdots, i_{m} ; i_{1}^{\prime}, i_{2}^{\prime}, \cdots, i_{m}^{\prime}\right\}}=<j\left|X_{i_{1}}^{+} X_{i_{2}}^{+} \cdots X_{i_{m}}^{+} X_{i_{m}^{\prime}}^{-} \cdots X_{i_{2}^{\prime}}^{-} X_{i_{1}^{\prime}}^{-}\right| j>
$$

for all $j$. Their coefficients depend on the components of the free fields $f_{i}^{ \pm}\left(z_{ \pm}\right)$, as they come out from the expansion of the exponentials. All such matrix elements can be computed recursively by shifting the $X_{i}^{+}$'s to the right, using the commutation relations of the Weyl generators, and the resulting expressions are polynomials of the Cartan matrix elements $K_{i j}$. For instance, we find

$$
\begin{aligned}
& D_{j}^{\left\{i ; i^{\prime}\right\}}=\delta_{i i^{\prime}} \delta_{i j}, \\
& D_{j}^{\left\{i_{1}, i_{2} ; i_{1}^{\prime}, i_{2}^{\prime}\right\}}=\delta_{i_{1} i_{1}^{\prime}} \delta_{i_{2} i_{2}^{\prime}} \delta_{i_{1}^{\prime} j}\left(2 \delta_{i_{2}^{\prime} j}-K_{i_{2}^{\prime} i_{1}^{\prime}}\right),
\end{aligned}
$$

and so on for all higher order terms. We also note, for completeness, that more general matrix elements of the form $D_{j}^{\left\{i_{1}, \cdots, i_{m} ; i_{1}^{\prime}, \cdots, i_{m^{\prime}}^{\prime}\right\}}$ arise as cross-terms from the expansion of $M_{+}^{-1}\left(z_{+}\right) M_{-}\left(z_{-}\right)$, but they are all zero, as it can be easily seen by direct computation, unless $m=m^{\prime}$, as in equation (3.11) above. Thus, all terms of the expansion can be determined entirely by the Lie algebra $\mathcal{G}$ and its highest weight representations, using free fields.

We summarize the results from a slightly different perspective that shows the power of integrability. Since $\lambda=0$ yields free field equations for the Toda variables, we may think of the power series expansion of the non-linear Toda fields as in perturbation theory,

$$
\phi_{i}\left(z_{+}, z_{-}\right)=\phi_{i}^{(0)}\left(z_{+}, z_{-}\right)+\lambda^{2} \phi_{i}^{(1)}\left(z_{+}, z_{-}\right)+\lambda^{4} \phi_{i}^{(2)}\left(z_{+}, z_{-}\right)+\cdots,
$$

where $\phi_{i}^{(0)}\left(z_{+}, z_{-}\right)$is a collection of two-dimensional free fields that arise for $\lambda=0$. The higher order correction terms $\phi_{i}^{(1)}\left(z_{+}, z_{-}\right), \phi_{i}^{(2)}\left(z_{+}, z_{-}\right), \cdots$ can be determined recursively in terms of the free fields $\phi_{i}^{(0)}\left(z_{+}, z_{-}\right)$, as it is usually done order by order in the parameter $\lambda^{2}$ using the Toda field equations with $\lambda \neq 0$. This is another way to say that the Toda field equations admit a free field realization, using the book keeping parameter $\lambda$. However, $\lambda$ is not a small parameter, as it can be rescaled at will. Therefore, computing only the first few terms in the perturbative expansion is not sufficient for extracting an approximate general solution. One has to determine all higher order terms in the Taylor series expansion, which is rather cumbersome algorithm to carry out in all detail. It is right here that the integrability of the Toda field equations saves the day, since all terms in the expansion can be computed systematically in terms of algebraic data, as above, and moreover, the entire series can be summed up in close form with the aid of the path-ordered exponential functions $M_{ \pm}\left(z_{ \pm}\right)$.

This remark concludes our brief discussion of the Toda field equations for simple Lie algebras $\mathcal{G}$ and makes us appreciate even more the algebraic description of their general solutions. Clearly, in all considerations, it is implicitly assumed that there are no nonperturbative corrections to the general configuration, which may then give rise to more 
exotic solutions of Toda theory; this is certainly true when the rank of $\mathcal{G}$ is finite, and we will assume that it is so in the infinite dimensional generalizations that are introduced next.

\subsection{Toda equations for continual Lie algebras}

The notion of continual Lie algebras arises naturally by considering a system of Weyl generators $\left\{H(t), X^{ \pm}(t)\right\}$, which depend on a continuous variable $t$ instead of a discrete Dynkin label, so that the commutation relations of the local part of the algebra assume the form [1], 12, 13.

$$
\begin{aligned}
& {\left[X^{+}(t), X^{-}\left(t^{\prime}\right)\right]=\delta\left(t-t^{\prime}\right) H\left(t^{\prime}\right), \quad\left[H(t), H\left(t^{\prime}\right)\right]=0,} \\
& {\left[H(t), X^{ \pm}\left(t^{\prime}\right)\right]= \pm K\left(t, t^{\prime}\right) X^{ \pm}\left(t^{\prime}\right) .}
\end{aligned}
$$

Here, $K\left(t, t^{\prime}\right)$ is a continual analogue of the Cartan matrix, which can be a distribution. The resulting algebras are infinite dimensional and one may also formally define their highest weight representations by introducing a normalized state $\mid t>$, so that

$$
X^{+}\left(t^{\prime}\right)|t>=0, \quad<t| X^{-}\left(t^{\prime}\right)=0, \quad H\left(t^{\prime}\right)\left|t>=\delta\left(t-t^{\prime}\right)\right| t>
$$

with $\langle t| t>=1$. This algebraic data will play an important role for the formulation of Toda field theories for all continual Lie algebras and the systematic construction of their general solution in terms of free fields, as in the finite dimensional case.

The general theory of continual Lie algebras involves the theory of distributions with values in Lie algebras, and therefore it is more natural to define them using the smeared form of the Weyl generators $\left\{H(\varphi), X^{ \pm}(\varphi)\right\}$ with respect to arbitrary functions $\varphi(t)$; we set, for notational purposes,

$$
A(\varphi)=\int d t \varphi(t) A(t)
$$

Then, the Cartan operator $K$ is a bilinear map on the vector space of all functions $\varphi$ and the commutation relations of the local part of the algebra, $\mathcal{G}_{-1} \oplus \mathcal{G}_{0} \oplus \mathcal{G}_{+1}$, assume the form

$$
\begin{aligned}
& {\left[X^{+}(\varphi), X^{-}(\psi)\right]=H(S(\varphi, \psi)), \quad[H(\varphi), H(\psi)]=0} \\
& {\left[H(\varphi), X^{ \pm}(\psi)\right]= \pm X^{ \pm}(K(\varphi, \psi))}
\end{aligned}
$$

where, $S(\varphi, \psi)=\varphi \cdot \psi$. More general continual Lie algebras can be constructed for other choices of the bilinear form $S$, which may differ from the identity; however, all pairs $(K, S)$ must satisfy the relation

$$
S(\varphi, K(\psi, \chi))=S(K(\psi, \phi), \chi)
$$

for consistency with the Jacobi identities. Then, if the operator $S$ is invertible, the substitution $H(S \varphi) \rightarrow H(\varphi)$ will bring the commutation relations of the algebra into a standard form,

$$
\tilde{K}=K S, \quad \tilde{S}=I
$$


Continual Lie algebras are denoted in general by $\mathcal{G}(K, S)$, by specifying the two bilinear maps $K, S$, and they are $Z$-graded algebras in the sense that

$$
\mathcal{G}(K, S)=\oplus_{n \in Z} \mathcal{G}_{n}
$$

where apart from the local part corresponding to $n=0$ and \pm 1 , the remaining elements of the algebra are constructed as usual, by taking successive commutators, so that $\mathcal{G}_{n}=$ $\left[\mathcal{G}_{n-1}, \mathcal{G}_{+1}\right]$ for all $n>0$ and $\mathcal{G}_{n}=\left[\mathcal{G}_{n+1}, \mathcal{G}_{-1}\right]$ for all $n<0$. Finally, an important concept is the dimension of the subspaces $\mathcal{G}_{n}$ relative to the dimension of the Cartan subalgebra $\mathcal{G}_{0}$,

$$
d_{n}=\operatorname{dim}_{\mathcal{G}_{0}} \mathcal{G}_{n}
$$

which varies from one example to another. We always have $d_{0}=d_{ \pm 1}=1$ by definition, but there are cases with $d_{n}=1$ for all $n$, i.e., algebras of constant growth, like ordinary simple Lie algebras including $\operatorname{sl}(\infty)$, and cases that exhibit polynomial growth in $n$; finally, there can be algebras with exponential growth, like the algebra that will be used for the systematic description of the renormalization group flows.

Using this abstract notion of continual Lie algebras, it is fairly easy to see, as a simple example, that the $s l(2)$-current algebra,

$$
\begin{aligned}
& {\left[X^{+}(\varphi), X^{-}(\psi)\right]=H(\varphi \cdot \psi), \quad[H(\varphi), H(\psi)]=0,} \\
& {\left[H(\varphi), X^{ \pm}(\psi)\right]= \pm 2 X^{ \pm}(\varphi \cdot \psi),}
\end{aligned}
$$

arises for the choice $K=2 I$ and $S=I$; all other current algebras can also be described in this fashion.

Another standard example is provided by the choice $K\left(t, t^{\prime}\right)=-\delta^{\prime \prime}\left(t-t^{\prime}\right)$, which can be thought as the continuous large $N$ limit of the Cartan matrix of the simple Lie algebras $s l(N)$, with $K_{i j}=2 \delta_{i, j}-\delta_{i+1, j}-\delta_{i, j+1}$. Thus, the Dynkin diagram of the continual Lie algebra $\operatorname{sl}(\infty)$ is a continuous line parametrized by the variable $t$, whereas for finite $N$ the skeletonization of $t$ yields the standard discrete Dynkin diagram of $\operatorname{sl}(N)$. The resulting infinite dimensional algebra $s l(\infty)$ turns out to be isomorphic to the algebra of area preserving diffeomorphisms of a two-dimensional surface. Working with smeared generators, we choose the bilinear maps $K(\varphi, \psi)=i \varphi^{\prime} \cdot \psi$ and $S(\varphi, \psi)=-i(\varphi \cdot \psi)^{\prime}$, which is same as having $\tilde{K}(\varphi, \psi)=-\varphi^{\prime \prime} \cdot \psi$ and $\tilde{S}(\varphi, \psi)=\varphi \cdot \psi$, so that $\tilde{K}\left(t, t^{\prime}\right)=-\delta^{\prime \prime}\left(t-t^{\prime}\right)$ and $\tilde{S}\left(t, t^{\prime}\right)=\delta\left(t-t^{\prime}\right)$ as above. Then, the complete structure of this continual Lie algebra can be worked out and it is described by the following system of commutation relations

$$
\left[X_{n}(\varphi), X_{m}(\psi)\right]=i X_{n+m}\left(m \varphi^{\prime} \psi-n \varphi \psi^{\prime}\right) ; \quad \text { for all } X_{n} \in \mathcal{G}_{n}
$$

Associating to each $X_{n}(\varphi)$ the mode functions $\varphi_{n}(t) e^{i n s}$, we see immediately that this algebra is isomorphic to the Poisson bracket algebra of all functions $\varphi(t, s)$ on a twodimensional surface (e.g., torus) with coordinates $(t, s)$, 12, 13, 42. Thus, $\operatorname{sl}(\infty)$ is an infinite dimensional Lie algebra with constant growth, since $d_{n}=1$ for all $n$; this is not always true, however, for other continual Lie algebras. 
There is a natural generalization of Toda field theory to continual Lie algebras provided that one introduces a "master" field $\Phi\left(z_{+}, z_{-} ; t\right)$ instead of a discrete collection of two-dimensional Toda fields $\phi_{i}\left(z_{+}, z_{-}\right)$. Then, the continual analogue of the Toda equations is given by [11, 12, 13, 43, 44]

$$
\partial_{+} \partial_{-} \Phi\left(z_{+}, z_{-} ; t\right)=\lambda^{2} \int d t^{\prime} K\left(t^{\prime}, t\right) e^{\Phi\left(z_{+}, z_{-} ; t^{\prime}\right)},
$$

which defines an integrable equation, as in the ordinary case, but lives in higher dimensions; however, this equation is not relativistic in higher dimensions, but only in the two dimensional space with light-cone coordinates $\left(z_{+}, z_{-}\right)$. Then, in analogy with Toda theories for finite dimensional Lie algebras, the continual analogue of the field equations follow from the two-dimensional zero curvature condition,

$$
\left[\partial_{+}+A_{+}, \partial_{-}+A_{-}\right]=0,
$$

where the gauge connections $A_{ \pm}\left(z_{+}, z_{-}\right)$take values in the local part of the Lie algebra, $\mathcal{G}_{-1} \oplus \mathcal{G}_{0} \oplus \mathcal{G}_{+1}$, with

$$
A_{+}=H(\Psi)+\lambda X^{+}(1), \quad A_{-}=\lambda X^{-}\left(e^{\Phi}\right) .
$$

These choices are straightforward generalizations of the ansatz (3.2) used for the case of finite dimensional algebras.

For any continual Lie algebra $\mathcal{G}(K, S)$, the resulting equations take the form

$$
\partial_{-} \Psi=\lambda^{2} S\left(1, e^{\Phi}\right), \quad \partial_{+} e^{\Phi}=K\left(\Psi, e^{\Phi}\right) .
$$

Then, for the special choice of bilinear maps, which will be implicitly assumed in all subsequent cases,

$$
K(\varphi, \psi)=(K \varphi) \cdot \psi, \quad S(\varphi, \psi)=S(\varphi \cdot \psi),
$$

and which describe $K$ and $S$ in terms of a much simpler set of linear maps, the system reduces to the continual Toda field equation

$$
\partial_{+} \partial_{-} \Phi=\lambda^{2} \tilde{K}(\exp \Phi)
$$

by elimination of the variable $\Psi$, or equivalently by making the consistent choice $\Psi=$ $K^{-1}\left(\partial_{t} \Phi\right)$, as in the finite dimensional case. Here, $\tilde{K}=K S$ as before, but the tilde can be dropped for all practical purposes.

A particularly interesting list of equations, which are closely related to each other in various ways, is provided by the following choices of Cartan kernel $K\left(t, t^{\prime}\right)$, having $S\left(t, t^{\prime}\right)=\delta\left(t-t^{\prime}\right)$ in all cases:

$$
\begin{array}{ll}
K\left(t, t^{\prime}\right)=2 \delta\left(t-t^{\prime}\right): & \partial_{+} \partial_{-} \Phi\left(z_{+}, z_{-} ; t\right)=2 \lambda^{2} e^{\Phi\left(z_{+}, z_{-} ; t\right)}, \\
K\left(t, t^{\prime}\right)=\delta^{\prime}\left(t-t^{\prime}\right): & \partial_{+} \partial_{-} \Phi\left(z_{+}, z_{-} ; t\right)=-\lambda^{2} \frac{\partial}{\partial t} e^{\Phi\left(z_{+}, z_{-} ; t\right)}, \\
K\left(t, t^{\prime}\right)=-\delta^{\prime \prime}\left(t-t^{\prime}\right): & \partial_{+} \partial_{-} \Phi\left(z_{+}, z_{-} ; t\right)=-\lambda^{2} \frac{\partial^{2}}{\partial t^{2}} e^{\Phi\left(z_{+}, z_{-} ; t\right)} .
\end{array}
$$


The first equation, which is associated to the $s l(2)$-current algebra, is just the Liouville equation for which $t$ acts as spectator; its geometric interpretation is well known from the 19th century and provides the condition for constant curvature two-dimensional metrics in the conformal gauge,

$$
d s^{2}=e^{\Phi\left(z_{+}, z_{-}\right)} d z_{+} d z_{-} .
$$

Positive or negative curvature spaces correspond to $\lambda^{2}=-1$ or +1 respectively. The third equation, which is associated to the algebra $s l(\infty)$, is the so called heavenly equation and arose in more recent years as the hyper-Kähler condition for four-dimensional metrics with a rotational (i.e., non-triholomorphic) isometry generated by a vector field $\partial_{\tau}$, using the system of adapted coordinates [45, 46, 47, 48,

$$
d s^{2}=\frac{1}{\partial_{t} \Phi}\left(d \tau \pm i\left(\partial_{+} \Phi\right) d z_{+} \mp i\left(\partial_{-} \Phi\right) d z_{-}\right)^{2}+\left(\partial_{t} \Phi\right)\left(e^{\Phi} d z_{+} d z_{-}+d t^{2}\right)
$$

for $\lambda^{2}=1$. Finally, the second equation is the one that describes the geometric deformations of two-dimensional metrics under the renormalization group flow in the conformal gauge for $\lambda^{2}=-1$. It will be investigated separately in all detail in section 3.3.

Straightforward generalization of the group theoretical framework that describes the solutions of Toda field equations in terms of arbitrary free field configurations yields the expression [1]

$$
\Phi\left(z_{+}, z_{-} ; t\right)=\Phi_{0}\left(z_{+}, z_{-} ; t\right)-\int d t^{\prime} K\left(t^{\prime}, t\right) \log <t^{\prime}\left|M_{+}^{-1}\left(z_{+}\right) M_{-}\left(z_{-}\right)\right| t^{\prime}>,
$$

where

$$
\Phi_{0}\left(z_{+}, z_{-} ; t\right)=f^{+}\left(z_{+} ; t\right)+f^{-}\left(z_{-} ; t\right)
$$

is a "master" free field in two dimensions, i.e., $\partial_{+} \partial_{-} \Phi_{0}\left(z_{+}, z_{-} ; t\right)=0$, which depends on the continuous variable $t$ rather than a discrete index $i$, as in ordinary Toda theory. Furthermore,

$$
M_{ \pm}\left(z_{ \pm} ; t\right)=\mathcal{P} \exp \left(\lambda \int^{z_{ \pm}} d z_{ \pm}^{\prime} \int^{t} d t^{\prime} e^{f^{ \pm}\left(z_{ \pm}^{\prime} ; t^{\prime}\right)} X^{ \pm}\left(t^{\prime}\right)\right)
$$

are path-ordered exponentials that generalize the corresponding expressions of Toda field theories to the case of continual Lie algebras in an obvious way, and the matrix elements $<t\left|M_{+}^{-1}\left(z_{+}\right) M_{-}\left(z_{-}\right)\right| t>$ can be computed using the continual (but formal) generalization of highest weight representations to the corresponding infinite dimensional Lie algebras.

The path-ordered exponentials can be expanded in infinite power series and each term can be determined algebraically in terms of the special matrix elements, as before,

$$
D_{t}^{\left\{t_{1}, t_{2}, \cdots, t_{m} ; t_{1}^{\prime}, t_{2}^{\prime}, \cdots, t_{m}^{\prime}\right\}}=<t\left|X^{+}\left(t_{1}\right) X^{+}\left(t_{2}\right) \cdots X^{+}\left(t_{m}\right) X^{-}\left(t_{m}^{\prime}\right) \cdots X^{-}\left(t_{2}^{\prime}\right) X^{-}\left(t_{1}^{\prime}\right)\right| t>,
$$

which involve an equal number of $X^{+}$'s and $X^{-}$'s. Then, using the commutation relations among the Weyl generators of the Lie algebra, one may easily derive recursive relations for these matrix elements, namely

$$
D_{t}^{\left\{t_{1}, t_{2}, \cdots, t_{m} ; t_{1}^{\prime}, t_{2}^{\prime}, \cdots, t_{m}^{\prime}\right\}}=\sum_{j=1}^{m} \delta\left(t_{m}-t_{j}^{\prime}\right)\left(\delta\left(t-t_{j}^{\prime}\right)-\sum_{k=1}^{j-1} K\left(t_{j}^{\prime}, t_{k}^{\prime}\right)\right) D_{t}^{\left\{t_{1}, \cdots, t_{m-1} ; t_{1}^{\prime}, \cdots, t_{j}^{\prime}, \cdots, t_{m}^{\prime}\right\}},
$$


with $D_{t}^{\left\{t_{1} ; t_{1}^{\prime}\right\}}=\delta\left(t-t_{1}\right) \delta\left(t-t_{1}^{\prime}\right)$. Here, $\hat{t}_{j}^{\prime}$ is used to denote that $t_{j}^{\prime}$ has been omitted by contraction with the index $t_{m}$. Although it is not possible to iterate the recursive relations in favor of producing a closed formula for the general matrix elements involved in the expansion, even in the simplest possible cases where the Cartan kernel has derivatives of the delta function, the first few terms of the expansion can be obtained explicitly starting with the normalization $\langle t \mid t\rangle=1$. More details will be given later for the special Lie algebra with $K\left(t, t^{\prime}\right)=\delta^{\prime}\left(t-t^{\prime}\right)$. In any case, this procedure provides a systematic description of the general solutions to Toda field equations for any continual Lie algebra in terms of free fields.

A practical question concerning the validity of the general solution is the convergence of the formal power series expansion, or else what is the rigorous meaning of the group elements $M_{ \pm}\left(z_{ \pm}\right)$that sum up the infinite many terms into the matrix elements $<t\left|M_{+}^{-1}\left(z_{+}\right) M_{-}\left(z_{-}\right)\right| t>$. Note that the path-ordered exponentials $M_{ \pm}\left(z_{ \pm}\right)$correspond to specific group elements obtained by exponentiation of the Lie algebra elements in $\mathcal{G}_{ \pm 1}$, with the free fields $\exp f_{i}^{ \pm}$playing the role of their canonical parameters. They are all well defined for finite dimensional simple Lie algebras, but for infinite dimensional algebras some appropriate regularization might be needed. Also, there might be solutions that admit free field realizations in patches, as one single patch $\left(f^{+}, f^{-}\right)$might not be sufficient for their construction ${ }^{3}$. A more rigorous definition of the group elements and their action on the highest weight states $\mid t>$, which are only formally described, is certainly required from a mathematical point of view. In general, the investigation of the convergence properties of the power series expansion in terms of free fields may depend crucially on the properties of the Cartan kernel $K\left(t, t^{\prime}\right)$. It is natural to expect that algebras of finite growth, i.e., $d_{n}<\infty$, will exhibit the necessary convergence properties, but it is fair to say that there is no systematic understanding of this issue at the moment.

\subsection{Renormalization group flows as Toda system}

Next, we specialize the general discussion to the continual Lie algebra with $K\left(t, t^{\prime}\right)=$ $\delta^{\prime}\left(t-t^{\prime}\right)$ and $S\left(t, t^{\prime}\right)=\delta\left(t-t^{\prime}\right)$, which is appropriate for the systematic description of the renormalization group flows of two-dimensional metrics as Toda field equations. According to the general theory, the local part of the algebra, $\mathcal{G}_{-1} \oplus \mathcal{G}_{0} \oplus \mathcal{G}_{+1}$, is described by the system of Weyl relations that are conveniently written here in smeared form,

$$
\begin{aligned}
& {\left[X_{+1}(\varphi), X_{-1}(\psi)\right]=H(\varphi \psi), \quad[H(\varphi), H(\psi)]=0} \\
& {\left[H(\varphi), X_{ \pm 1}(\psi)\right]=\mp X_{ \pm 1}\left(\varphi^{\prime} \psi\right)}
\end{aligned}
$$

The remaining part of the full $Z$-graded algebra $\mathcal{G}=\oplus_{n \in Z} \mathcal{G}_{n}$ is generated recursively, as usual, by taking successive commutators of $X_{ \pm 1}$. We do not need to know it for the

\footnotetext{
${ }^{3} \mathrm{~A}$ characteristic example of this type is provided by the Atiyah-Hitchin metric, which is a hyperKähler metric with rotational isometry that solves equation (3.32) for the algebra $s l(\infty)$ with $K\left(t, t^{\prime}\right)=$ $-\delta^{\prime \prime}\left(t-t^{\prime}\right)$ (see, for instance, 48] and references therein).
} 
purposes of the proposed association, but we will collect, nevertheless, everything that is known about the structure of the full algebra at the end of this section, focusing on the proof of its exponential growth.

Consider the zero curvature condition $\left[\partial_{+}+A_{+}\left(z_{+}, z_{-}\right), \partial_{-}+A_{-}\left(z_{+}, z_{-}\right)\right]=0$ and make the most general ansatz for the gauge connections $A_{ \pm}\left(z_{+}, z_{-}\right) \in \mathcal{G}_{0} \oplus \mathcal{G}_{ \pm 1}$,

$$
A_{ \pm}\left(z_{+}, z_{-}\right)=H\left(u_{ \pm}\right)+\lambda X_{ \pm 1}\left(f_{ \pm}\right)
$$

where $u_{ \pm}$and $f_{ \pm}$are all functions of $\left(z_{+}, z_{-} ; t\right)$. Then, by comparing terms of the Lie algebra elements $H$ and $X_{ \pm 1}$ that arise in this case, we find the following system of equations

$$
\begin{aligned}
& \frac{\partial u_{+}}{\partial t}=-\partial_{+}\left(\log f_{-}\right), \quad \frac{\partial u_{-}}{\partial t}=\partial_{-}\left(\log f_{+}\right), \\
& \partial_{+} u_{-}-\partial_{-} u_{+}+\lambda^{2} f_{+} f_{-}=0 .
\end{aligned}
$$

Obviously, $u_{ \pm}$can be easily eliminated by taking appropriate derivatives of these equations to yield

$$
\partial_{+} \partial_{-} \Phi\left(z_{+}, z_{-} ; t\right)=-\lambda^{2} \frac{\partial}{\partial t} e^{\Phi\left(z_{+}, z_{-} ; t\right)}, \quad \text { with } \Phi=\log \left(f_{+} f_{-}\right)
$$

as the continual Toda field equation associated with the Lie algebra $\mathcal{G}(d / d t ; 1)$. It describes the renormalization group flow of two-dimensional sigma models in a conformally flat system of target space coordinates $\left(z_{+}, z_{-}\right)$, as advertised, with $\lambda^{2}=-1$.

Note that our ansatz appears to be slightly more general than the ansatz (3.26) used in section 3.2 , but this is not really so, as it reflects the gauge arbitrariness of the zero curvature formulation of our equations. In fact, it can be easily seen that one can set $u_{-}=0$ by performing a gauge transformation $A_{ \pm} \rightarrow g^{-1}\left(\partial_{ \pm}+A_{ \pm}\right) g$ with $g=\exp (H(\varphi))$ and $\partial_{-} \varphi=-u_{-}$. The function $\Phi=\log \left(f_{+} f_{-}\right)$is gauge invariant, and, as a result, we may set $f_{+}=1, f_{-}=\exp \Phi$ in order to bring the general ansatz into a more simple and standard form. In any case, it casts the renormalization group flows into a zero curvature form with the aid of the infinite dimensional algebra $\mathcal{G}(d / d t ; 1)$, which is thus an integrable system ${ }^{4}$.

We may take advantage of the reformulation of these equations as Toda system in order to apply the algorithm for the systematic description of the general solution in terms of a much simpler family of two-dimensional free field configurations $f^{ \pm}\left(z_{ \pm} ; t\right)$, given by

$$
\Phi\left(z_{+}, z_{-} ; t\right)=\Phi_{0}\left(z_{+}, z_{-} ; t\right)+\partial_{t}\left(\log <t\left|M_{+}^{-1}\left(z_{+} ; t\right) M_{-}\left(z_{-} ; t\right)\right| t>\right),
$$

\footnotetext{
${ }^{4}$ However, the notion of integrability should be used with care when the gauge connections $A_{ \pm}$take values in infinite dimensional Lie algebras, because in some cases the growth of the algebra can be large and there might not be a straightforward way to construct and count the integrals of motion; we will make a few extra comments about it later, while discussing the exponential growth of $\mathcal{G}(d / d t ; 1)$.
} 
by specializing equations (3.35), (3.36) and (3.37) to $\mathcal{G}(d / d t ; 1)$ with $\lambda=i$, so that $\lambda^{2}=-1$. Then, expanding the path ordered exponentials in $M_{ \pm}\left(z_{ \pm} ; t\right)$, we obtain the series

$$
\begin{aligned}
&<t \mid M_{+}^{-1} M_{-} \mid t>=1+\sum_{m=1}^{\infty} \int^{z_{+}} d z_{1}^{+} \cdots \int^{z_{m-1}^{+}} d z_{m}^{+} \int^{z_{-}} d z_{1}^{-} \cdots \int^{z_{m-1}^{-}} d z_{m}^{-} \times \\
& \times \int \prod_{i=1}^{m} d t_{i} \int \prod_{i=1}^{m} d t_{i}^{\prime} \exp f^{+}\left(z_{i}^{+} ; t_{i}\right) \exp f^{-}\left(z_{i}^{-} ; t_{i}^{\prime}\right) D_{t}^{\left\{t_{1}, \cdots, t_{m} ; t_{1}^{\prime}, \cdots, t_{m}^{\prime}\right\}}
\end{aligned}
$$

with the ordering $z_{ \pm} \geq z_{1}^{ \pm} \cdots \geq z_{m-1}^{ \pm} \geq z_{m}^{ \pm}$. The elements $D_{t}^{\left\{t_{1}, t_{2}, \cdots, t_{m} ; t_{1}^{\prime}, t_{2}^{\prime}, \cdots, t_{m}^{\prime}\right\}}$ follow from the recursive relations (3.39) for $K\left(t, t^{\prime}\right)=\delta^{\prime}\left(t-t^{\prime}\right)$, and the result turns out to be

$$
\begin{aligned}
& D_{t}^{\left\{t_{1} ; t_{1}^{\prime}\right\}}=\delta\left(t-t_{1}\right) \delta\left(t_{1}-t_{1}^{\prime}\right) \\
& D_{t}^{\left\{t_{1}, t_{2} ; t_{1}^{\prime}, t_{2}^{\prime}\right\}}=\delta\left(t-t_{1}\right) \delta\left(t_{1}-t_{1}^{\prime}\right) \delta\left(t_{2}-t_{2}^{\prime}\right)\left(2 \delta\left(t-t_{2}\right)+\partial_{t} \delta\left(t-t_{2}\right)\right), \\
& D_{t}^{\left\{t_{1}, t_{2}, t_{3} ; t_{1}^{\prime}, t_{2}^{\prime}, t_{3}^{\prime}\right\}}=\delta\left(t-t_{1}\right) \delta\left(t-t_{3}\right) \delta\left(t_{1}-t_{1}^{\prime}\right) \delta\left(t_{2}-t_{3}^{\prime}\right) \delta\left(t_{3}-t_{2}^{\prime}\right) \times \\
& \quad \times\left(2 \delta\left(t-t_{2}\right)+\partial_{t} \delta\left(t-t_{2}\right)\right)+ \\
& +\delta\left(t-t_{1}\right) \delta\left(t_{1}-t_{1}^{\prime}\right) \delta\left(t_{2}-t_{3}^{\prime}\right) \delta\left(t_{3}-t_{2}^{\prime}\right) \times \\
& \quad \times\left(\delta\left(t-t_{3}\right)+\partial_{t} \delta\left(t-t_{3}\right)\right)\left(2 \delta\left(t-t_{2}\right)+\partial_{t} \delta\left(t-t_{2}\right)\right)+ \\
& + \\
& \quad \delta\left(t-t_{1}\right) \delta\left(t_{1}-t_{1}^{\prime}\right) \delta\left(t_{2}-t_{2}^{\prime}\right) \delta\left(t_{3}-t_{3}^{\prime}\right) \times \\
& \quad \times\left(2 \delta\left(t-t_{2}\right)+\partial_{t} \delta\left(t-t_{2}\right)\right)\left(\delta\left(t-t_{3}\right)+\partial_{t} \delta\left(t-t_{3}\right)+\partial_{t_{2}} \delta\left(t_{2}-t_{3}\right)\right)
\end{aligned}
$$

and so on. Thus, we obtain a power series expansion of the non-linear field $\Phi\left(z_{+}, z_{-} ; t\right)$ in terms of free fields, as advertised before, but its structure becomes messy rather quickly.

The families of the two-dimensional chiral fields $f^{ \pm}$, with $\partial_{\mp} f^{ \pm}\left(z_{ \pm} ; t\right)=0$, can be thought as providing a natural system of flat coordinates in the infinite dimensional space of all two-dimensional metrics, where the renormalization group flows act. Every physical trajectory in superspace corresponds to all possible geometric deformations of a given sigma model, which, according to our formalism, it can be solely described by the dependence of the parameters $f^{ \pm}\left(z_{ \pm} ; t\right)$ on $t$. However, the convergence of the infinite series (3.45) cannot be easily proved in the strict mathematical sense, and one might question its validity, as well as its practical use, for solving the renormalization group equations. We will encounter some simple algebraic solutions of the continual Toda field equation later, while studying the renormalization group flows of constant curvature metrics (with positive or negative curvature), which describe axially symmetric deformations of their volume and shape. These special examples, which are mini-superspace approximations of the more general trajectories in superspace, will be used as a guide for testing the validity of the formal power series expansion (3.45) that describes $\Phi\left(z_{+}, z_{-} ; t\right)$ in free field coordinates. Explicit comparison will be made in those cases by first extracting the form of the corresponding functions $f^{ \pm}\left(z_{ \pm} ; t\right)$, and then checking (order by order) the terms that arise in the power series expansion of the given solution. A similar investiga- 
tion has been carried out before for the continual Lie algebra $\mathcal{G}\left(-d^{2} / d t^{2} ; 1\right)$ that arises in problems of four-dimensional hyper-Kähler geometry.

In view of future applications, we describe the simplifications that occur in the power series expansion (3.45) for the special class of free field configurations

$$
\Phi_{0}\left(z_{+}, z_{-} ; t\right)=c \cdot\left(z_{+}+z_{-}\right)+d(t) \equiv c Y+d(t)
$$

which are used to parametrize solutions of the Toda field equations with an isometry, i.e., solutions which are independent of $z_{+}-z_{-}$. Here, $c$ is taken to be an arbitrary constant and $d(t)$ is an arbitrary function of $t$. Then, the first few terms in the power series expansion (3.45) can be easily computed and the result reads

$$
\begin{aligned}
<t\left|M_{+}^{-1} M_{-}\right| t> & =1+\frac{1}{c^{2}} e^{\Phi_{0}}+\frac{1}{4 c^{4}} e^{\Phi_{0}}\left(2 e^{\Phi_{0}}+\partial_{t} e^{\Phi_{0}}\right)+ \\
& +\frac{1}{36 c^{6}} e^{\Phi_{0}}\left(6 e^{2 \Phi_{0}}+9 e^{\Phi_{0}} \partial_{t} e^{\Phi_{0}}+3\left(\partial_{t} e^{\Phi_{0}}\right)^{2}+e^{\Phi_{0}} \partial_{t}^{2} e^{\Phi_{0}}\right)+\cdots
\end{aligned}
$$

Subsequently, using the identity $\log (1+x)=x-x^{2} / 2+x^{3} / 3-\cdots$, it can be shown that $\Phi\left(z_{+}, z_{-} ; t\right)$ takes the form

$$
\Phi=\Phi_{0}+\frac{1}{c^{2}} \partial_{t} e^{\Phi_{0}}+\frac{1}{4 c^{4}} \partial_{t}\left(e^{\Phi_{0}} \partial_{t} e^{\Phi_{0}}\right)+\frac{1}{36 c^{6}} \partial_{t}\left(3 e^{\Phi_{0}}\left(\partial_{t} e^{\Phi_{0}}\right)^{2}+e^{2 \Phi_{0}} \partial_{t}^{2} e^{\Phi_{0}}\right)+\cdots
$$

and provides a perturbative expansion of the Toda field around $\exp \Phi \simeq 0$, i.e., for $\Phi_{0} \rightarrow-\infty$.

We note finally that there other special solutions of the continual Toda field equation that arise by suitable embedding the general solution of the Liouville equation,

$$
e^{\Phi_{\mathrm{L}}\left(z_{+}, z_{-}\right)}=\frac{\partial_{+} F^{+}\left(z_{+}\right) \partial_{-} F^{-}\left(z_{-}\right)}{\left(1 \pm F^{+}\left(z_{+}\right) F^{-}\left(z_{-}\right)\right)^{2}} \quad \text { with } \quad \partial_{+} \partial_{-} \Phi_{\mathrm{L}}=\mp 2 e^{\Phi_{\mathrm{L}}}
$$

where the different signs correspond to positive or negative curvature spaces respectively. Here, to convey with the notation used earlier for the free field realization of Toda theories, we have chosen a pair of arbitrary holomorphic and anti-holomorphic functions $F^{ \pm}\left(z_{ \pm}\right)$ so that $F^{ \pm}\left(z_{ \pm}\right)=\int^{z_{ \pm}} d z_{ \pm}^{\prime} \exp f^{ \pm}\left(z_{ \pm}^{\prime}\right)$. Then, we have, in particular, the special class of solutions

$$
e^{\Phi\left(z_{+}, z_{-} ; t\right)}= \pm 2\left(t_{0}-t\right) e^{\Phi_{L}\left(z_{+}, z_{-}\right)}
$$

with $t_{0}$ being an arbitrary constant, which admit a very simple free field realization. These special solutions have an interesting geometric interpretation, as we will see later; we will call Liouville lines all the trajectories of the renormalization group flow that correspond to these special solutions, provided that $\pm\left(t_{0}-t\right) \geq 0$ for real $\Phi$.

\subsection{Novelties of the Lie algebra $\mathcal{G}(d / d t ; 1)$}

We end this section by discussing two novel features of the bosonic Lie algebra $\mathcal{G}(d / d t ; 1)$, which make it rather unique from the mathematical and physical point of view. 


\section{Supersymmetric Toda field equations}

First, there is a remarkable connection between the continual Toda field theories associated to the Cartan kernels $K\left(t, t^{\prime}\right)=\delta^{\prime}\left(t-t^{\prime}\right)$ and $K\left(t, t^{\prime}\right)=-\delta^{\prime \prime}\left(t-t^{\prime}\right)$, which can be high-lighted with the aid of supersymmetric generalizations of Toda systems. This connection also shows that $\delta^{\prime}\left(t-t^{\prime}\right)$, which is anti-symmetric, has a natural interpretation as the Cartan kernel of a continual superalgebra, but it is rather peculiar for bosonic Lie algebra like $\mathcal{G}(d / d t ; 1)$.

Consider the simple Lie superalgebra $s l(N \mid N+1)$ for which the system of simple roots is odd and corresponds to the super-principal embedding of $\operatorname{osp}(1 \mid 2)$ in $\operatorname{sl}(N \mid N+1)$, as depicted in figure 2 below that represents its Dynkin diagram in Kac's notation [49] (but see also [50] for a comprehensive exposition).

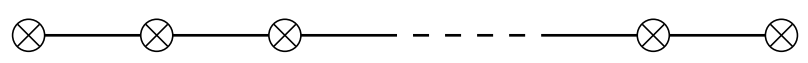

Figure 2: Simple odd roots of the Lie superalgebra $\operatorname{sl}(N \mid N+1)$.

Then, the continuous large $N$ limit of the Lie superalgebra $\operatorname{sl}(N \mid N+1)$ is naturally defined by the following relations among its Weyl generators,

$$
\begin{aligned}
& {\left[H(\varphi), X_{ \pm 1}(\psi)\right]_{-}=\mp X_{ \pm 1}\left(\varphi^{\prime} \psi\right), \quad[H(\varphi), H(\psi)]_{-}=0,} \\
& {\left[X_{+1}(\varphi), X_{-1}(\psi)\right]_{+}=H(\varphi \cdot \psi) .}
\end{aligned}
$$

Here, $H(\varphi)$ is an even element, $X_{ \pm 1}(\varphi)$ are odd elements, whereas the Cartan kernel, which arises as the continuous limit of the Cartan matrix of $s l(N \mid N+1), K_{i j}=\delta_{i+1, j}-$ $\delta_{i, j+1}$, is chosen anti-symmetric and coincides with $K\left(t, t^{\prime}\right)=\delta^{\prime}\left(t-t^{\prime}\right)$. As a result, the local part of this superalgebra looks the same as for the contragradient continual Lie algebra $\mathcal{G}(d / d t ; 1)$, but now the elements $X_{ \pm 1}(\varphi)$ are odd and satisfy anti-commutation (rather than commutation) relations.

The Toda field equation associated to the continual Lie superalgebra $\operatorname{sl}(\infty \mid \infty+1)$ is best described using a superfield $\hat{\Phi}\left(z_{ \pm} ; \theta_{ \pm} ; t\right)$ which is defined in $2 / 2$-dimensional superspace with two even $\left(z_{ \pm}\right)$and two odd $\left(\theta_{ \pm}\right)$coordinates. We have the supersymmetric Toda field equation [51]

$$
D_{+} D_{-} \hat{\Phi}\left(z_{ \pm} ; \theta_{ \pm} ; t\right)=\frac{\partial}{\partial t} e^{\hat{\Phi}\left(z_{ \pm} ; \theta_{ \pm} ; t\right)},
$$

where the supersymmetric covariant derivatives in superspace are defined, as usual,

$$
D_{ \pm}=\frac{\partial}{\partial \theta_{ \pm}}+i \theta_{ \pm} \frac{\partial}{\partial z_{ \pm}}
$$

In analogy with the non-supersymmetric case, the Toda field equation for the superfield $\hat{\Phi}\left(z_{ \pm} ; \theta_{ \pm} ; t\right)$ can be obtained from the zero curvature condition

$$
\left[D_{+}+A_{+}, D_{-}+A_{-}\right]_{+}=0,
$$


using the odd gauge connections

$$
A_{+}=H\left(D_{+} \hat{\Psi}\right)+X_{+}(1), \quad A_{-}=X_{-}\left(e^{\hat{\Phi}}\right), \quad \text { with } \hat{\Phi}=\frac{\partial \hat{\Psi}}{\partial t}
$$

Parametrizing the continual Toda superfield as

$$
\hat{\Phi}\left(z_{ \pm} ; \theta_{ \pm} ; t\right)=\Phi\left(z_{ \pm} ; t\right)+\theta_{+} \Omega_{+}\left(z_{ \pm} ; t\right)+\theta_{-} \Omega_{-}\left(z_{ \pm} ; t\right)+\theta_{+} \theta_{-} F\left(z_{ \pm} ; t\right)
$$

we may write down the field equations in terms of components. This is achieved by eliminating the auxiliary field $F\left(z_{ \pm} ; t\right)$, as usual, using the identification $F=\partial(\exp \Phi) / \partial t$, in which case the resulting equations read

$$
\begin{aligned}
& \partial_{+} \Omega_{+}=-i \frac{\partial}{\partial t}\left(e^{\Phi} \Omega_{-}\right), \quad \partial_{-} \Omega_{-}=i \frac{\partial}{\partial t}\left(e^{\Phi} \Omega_{+}\right), \\
& \partial_{+} \partial_{-}(2 \Phi)=\frac{\partial^{2}}{\partial t^{2}} e^{2 \Phi}-\frac{\partial}{\partial t}\left(e^{\Phi} \Omega_{+} \Omega_{-}\right) .
\end{aligned}
$$

Setting $\Omega_{+}=0=\Omega_{-}$, we arrive at the continual Toda field equation (3.32) for the infinite dimensional Lie algebra $\mathcal{G}\left(-d^{2} / d t^{2} ; 1\right) \simeq s l(\infty)$, which is just the bosonic part of the superalgebra $\operatorname{sl}(\infty \mid \infty+1)$.

Thus, we find that the anti-symmetric Cartan kernel $K\left(t, t^{\prime}\right)=\delta^{\prime}\left(t-t^{\prime}\right)$ is naturally associated to the superalgebra of area preserving diffeomorphisms by giving a suitable geometric interpretation to $\operatorname{sl}(\infty \mid \infty+1)$, as for $\operatorname{sl}(\infty)$. We do not know whether this fact plays a deeper role in understanding the structure of the bosonic Lie algebra $\mathcal{G}(d / d t ; 1)$ that describes the renormalization group flows in two-dimensional sigma models. For, if we were defining the elements of the Cartan matrix as inner product of the simple roots, $K_{i j}=\vec{a}_{i} \cdot \vec{a}_{j}$, the roots $\vec{a}_{i}$ would have been fermionic by the anti-symmetry of $K$. In the continual case, in particular, $\vec{a}(t)$ would correspond to a one-dimensional fermion. Bosonic Lie algebras with such root systems have not been studied before in the mathematics literature, to the best of our knowledge, and they certainly lead to rather exotic structures.

\section{Exponential growth and related topics}

The bosonic Lie algebra $\mathcal{G}(d / d t ; 1)$ exhibits another remarkable property, which can only be seen by going beyond the local part $\mathcal{G}_{-1} \oplus \mathcal{G}_{0} \oplus \mathcal{G}_{+1}$ associated to the system of Weyl generators

$$
\begin{aligned}
& {\left[X_{+1}(\varphi), X_{-1}(\psi)\right]=H(\varphi \psi), \quad[H(\varphi), H(\psi)]=0} \\
& {\left[H(\varphi), X_{ \pm 1}(\psi)\right]=\mp X_{ \pm 1}\left(\varphi^{\prime} \psi\right)}
\end{aligned}
$$

The full algebra is a $Z$-graded algebra $\mathcal{G}=\oplus_{n \in Z} \mathcal{G}_{n}$, which according to the general theory can be generated recursively by taking successive commutators of $X_{ \pm 1}$. The generators of $\mathcal{G}_{ \pm 2}$ are defined, as usual, by $X_{ \pm 2} \in\left[\mathcal{G}_{ \pm 1}, \mathcal{G}_{ \pm 1}\right]$, and their commutation relations assume the form

$$
\begin{aligned}
& {\left[X_{ \pm 1}(\varphi), X_{ \pm 1}(\psi)\right]= \pm X_{ \pm 2}\left(\varphi^{\prime} \psi-\varphi \psi^{\prime}\right), \quad\left[H(\varphi), X_{ \pm 2}(\psi)\right]= \pm 2 X_{ \pm 2}\left(\varphi^{\prime} \psi\right)} \\
& {\left[X_{ \pm 2}(\varphi), X_{\mp 1}(\psi)\right]= \pm X_{ \pm 1}(\varphi \psi), \quad\left[X_{+2}(\varphi), X_{-2}(\psi)\right]=0}
\end{aligned}
$$


They can be easily derived by requiring consistency with the Jacobi identities [13.

Proceeding further to $\mathcal{G}_{ \pm 3}$, one finds that there are two independent generators that parametrize each one of the Lie algebra elements $\left[\mathcal{G}_{ \pm 1}, \mathcal{G}_{ \pm 2}\right]$, which turn to be

$$
X_{ \pm 3}^{(s)}(\varphi)=2^{s-3}\left[X_{ \pm 1}(1), X_{ \pm 2}(\varphi)\right]+(-1)^{s}\left[X_{ \pm 1}(\varphi), X_{ \pm 2}(1)\right], \quad \text { for } s=1,2 .
$$

It can be verified that these combinations satisfy the commutation relations

$$
\begin{aligned}
& {\left[X_{ \pm 3}^{(1)}(\varphi), X_{\mp 1}(\psi)\right]=\mp X_{ \pm 2}(\varphi \psi), \quad\left[X_{ \pm 3}^{(2)}(\varphi), X_{\mp 1}(\psi)\right]= \pm X_{ \pm 2}\left((\varphi \psi)^{\prime}\right),} \\
& {\left[H(\varphi), X_{ \pm 3}^{(s)}(\psi)\right]= \pm 3 X_{ \pm 3}^{(s)}\left(\varphi^{\prime} \psi\right),}
\end{aligned}
$$

which, thus, prove the linear independence of the elements $X_{ \pm 3}^{(1)}$ and $X_{ \pm 3}^{(2)}[13$.

More generally, for any positive integer $n \geq 2$, it can be shown by induction that if $\mathcal{G}_{ \pm n}$ is spanned by the independent elements $X_{ \pm n}^{(1)}, \cdots, X_{ \pm n}^{\left(d_{n}\right)}$, the subspace $\mathcal{G}_{ \pm(n+1)}=$ $\left[\mathcal{G}_{ \pm 1}, \mathcal{G}_{ \pm n}\right]$ will be spanned by the following $2 d_{n}$ independent elements [13]

$$
\begin{aligned}
& X_{ \pm(n+1)}^{(s)}(\varphi)=\alpha_{n}^{(s)}\left[X_{ \pm 1}(1), X_{ \pm n}^{(s)}(\varphi)\right]-\left[X_{ \pm 1}(\varphi), X_{ \pm n}^{(s)}(1)\right] ; \quad 1 \leq s \leq d_{n} \\
& X_{ \pm(n+1)}^{(s)}(\varphi)=\beta_{n}^{(s)}\left[X_{ \pm 1}(1), X_{ \pm n}^{(s)}(\varphi)\right]+\left[X_{ \pm 1}(\varphi), X_{ \pm n}^{(s)}(1)\right] ; \quad d_{n}+1 \leq s \leq 2 d_{n}
\end{aligned}
$$

for some appropriately chosen constants $\alpha_{n}^{(s)}$ and $\beta_{n}^{(s)}$. Thus, the dimension of the subspaces $\mathcal{G}_{n}$ (relative to the dimension of $\mathcal{G}_{0}$, which is taken to be 1 ) is given by

$$
d_{0}=d_{ \pm 1}=1, \quad d_{n}=2^{|n|-2} \quad \text { for }|n| \geq 2
$$

and therefore, this particular continual algebra exhibits exponential growth that makes it rather exotic, but worth studying in great detail. It can also be shown that

$$
\left[\mathcal{G}_{ \pm 2}, X_{\mp(n+1)}^{(s)}\right]=0
$$

for all $n \geq 2$ and $1 \leq s \leq d_{n}$.

The complete set of commutation relations of this infinite dimensional algebra have not been derived systematically to this day and there has been no analogue of the corresponding Serre relations, to the best of our knowledge, due to its exponential growth. In fact, this is a typical behavior of indefinite Lie algebras, with hyperbolic algebras being the simplest, yet quite intractable, examples. Recall that hyperbolic algebras are usually defined using a symmetric Cartan matrix with Lorentzian structure in their root system 14], but here, the algebra $\mathcal{G}(d / d t ; 1)$, also has the additional feature that its Cartan kernel is anti-symmetric. Then, in analogy with hyperbolic algebras, one expects that the structure of the full algebra $\mathcal{G}(d / d t ; 1)$ can only be accommodated in string theory, but there is no clear way to proceed in this direction at the moment. Fortunately, we do not need to know the structure of the full algebra in order to describe the renormalization group flows of two-dimensional sigma models as Toda field equations, because we are only considering the simplest case of Toda field equations with both gauge connections $A_{ \pm}\left(z_{+}, z_{-}\right)$taking their values in the local part of the algebra, $\mathcal{G}_{-1} \oplus \mathcal{G}_{0} \oplus \mathcal{G}_{+1}$. The 
structure of the full algebra is only needed to describe the form of the conserved currents, which in ordinary Toda theory are constructed in terms of the Casimir operators [52, but they are not known yet in the present case. For this reason we are not in the position to provide a systematic list of all integrals of the renormalization group flows, despite their integrability. We postpone the construction of the conserved currents to future work with the note that unexpected results might arise, as for some Toda field theories associated to hyperbolic algebras [53. A few more comments will be made at the end of this paper.

It is worth mentioning now that generalized versions of the Toda field equations can be considered by introducing gauge connections $A_{ \pm}$taking values in the subspace $\mathcal{G}_{-N} \oplus \mathcal{G}_{-N+1} \oplus \cdots \oplus \mathcal{G}_{0} \oplus \cdots \oplus \mathcal{G}_{N-1} \oplus \mathcal{G}_{N}$ of the complete algebra for any finite, but fixed, value $N \geq 2$, i.e.,

$$
A_{ \pm}\left(z_{+}, z_{-}\right)=H\left(\varphi_{0}^{ \pm}\right)+\sum_{n=1}^{N} \sum_{s=1}^{d_{n}} X_{ \pm n}^{(s)}\left(\varphi_{n}^{ \pm ; s}\right) .
$$

Here, the gauge connection are parametrized by arbitrary functions $\varphi_{n}^{ \pm ; s}\left(z_{+}, z_{-} ; t\right)$ for each independent element that generates the subspaces $\mathcal{G}_{ \pm n}$. Thus, taking into account the exponential growth of the continual algebra $\mathcal{G}(d / d t ; 1)$, we see that $A_{+}$depends on $2^{N-1}+1$ such arbitrary functions, and likewise for the gauge connection $A_{-}$. Of course, one can always make a gauge transformation to set $\varphi_{0}^{-}=0$ in the zero curvature condition, in which case the generalized system of Toda field equations involves $2^{N}+1$ functions in total that satisfy the same number of non-linear equations. These equations follow by comparing terms for the generators $H$ and $\left\{X_{ \pm n}^{(s)} ; 1 \leq n \leq N, 1 \leq s \leq d_{n}\right\}$ that arise by computing the commutator $\left[A_{+}, A_{-}\right] \in \mathcal{G}_{-N} \oplus \cdots \oplus \mathcal{G}_{0} \oplus \cdots \oplus \mathcal{G}_{N}$ in the zero curvature condition.

This is precisely the way that the equation $\partial_{+} \partial_{-} \Phi=\partial_{t}(\exp \Phi)$ arose earlier for $N=1$, but for $N \geq 2$ the details of the generalized Toda system of field equations depend crucially on the structure of the commutation relations of the algebra, which is still lacking in all generality. For $N=2$, it is indeed possible to work out the details of the generalized system of Toda field equations using the explicit form of the commutation relations in the subspace $\mathcal{G}_{-2} \oplus \mathcal{G}_{-1} \oplus \mathcal{G}_{0} \oplus \mathcal{G}_{+1} \oplus \mathcal{G}_{+2}$ given above, but for $N \geq 3$ we cannot write down a systematic answer at this moment. In any case, it will be interesting to examine whether such generalized continual Toda field equations have any relevance to the beta function equations and the renormalization group flows of sigma models, where, apart from the metric, couplings to other fields are also allowed (see, for instance, [54, and references therein). We expect that this line of thought will help to clarify the full structure of the continual Lie algebra $\mathcal{G}(d / d t ; 1)$ and provide a string theoretic realization of it in two dimensions. 


\section{The sausage model}

There is a well studied solution of the continual Toda field equation associated with the renormalization group flow of two-dimensional spaces with spherical topology, $S^{2}$. Consider the class of axially symmetric sigma models with two-dimensional action

$$
S_{\mathrm{t}}=\frac{1}{2} \int e^{\Phi(Y ; t)}\left(\left(\partial_{\mu} X\right)^{2}+\left(\partial_{\mu} Y\right)^{2}\right) d^{2} w,
$$

where the conformal factor of the target space metric is given by the one-parameter family of functions $\Phi(Y ; t)$, 35],

$$
e^{\Phi(Y ; t)}=\frac{2}{a(t)+b(t) \cosh 2 Y} .
$$

Here, $X$ is an angular coordinate, $0 \leq X \leq 2 \pi$, which accounts for the axial symmetry of the metric that is independent of $X$, whereas $Y$ ranges over the whole real axis, $-\infty<Y<+\infty$. Furthermore, the end points $Y= \pm \infty$ are used to parametrize the north and the south poles of the manifold with spherical topology, where the boundary conditions

$$
\Phi(Y ; t) \sim-2|Y| \quad \text { for } Y \rightarrow \pm \infty,
$$

are imposed for regularity.

In general, the metric of the two-dimensional target space is real provided that $a \geq-b$ and $b \geq 0$, which is implicitly assumed here, but later we will impose the stronger condition $a \geq b \geq 0$ as more appropriate for the geometric deformations of the $O(3)$ sigma model. The target space is a deformed sphere with axial symmetry, rather than round sphere, and so the geometry looks like a sausage, in general, which accounts for the term "sausage model" used in the literature. Further justification will be given shortly by studying the geometric deformation of this class of models under the action of the renormalization group flows.

There are two special configurations that arise in the general class of sausage models and deserve special reference. First, the case $a(t)=b(t)=1$ corresponds to the usual $O(3)$ sigma model with the geometry of a round sphere associated to the conformal factor

$$
e^{\Phi(Y)}=\frac{1}{\cosh ^{2} Y} .
$$

Actually, to make contact with the standard description of the $O(3)$ sigma model, it is convenient to introduce two angular coordinates $(\theta, \varphi)$ with

$$
\cos \theta=\tanh Y, \quad \varphi=X,
$$

so that $0 \leq \theta \leq \pi$, as $Y$ ranges from $+\infty$ to $-\infty$, whereas $0 \leq \varphi \leq 2 \pi$. They provide a more natural parametrization of the two-dimensional sphere using the geographical chart $(\theta, \varphi)$ with the north and south poles located at $\theta=0$ and $\pi$ respectively. Then, the target space metric assumes the equivalent form

$$
d s^{2}=\frac{1}{2}\left(d \theta^{2}+\sin ^{2} \theta d \varphi^{2}\right)
$$


and describes a round sphere of radius $1 / \sqrt{2}$; clearly, the beta function is non-vanishing in this case. Another special configuration in the one-parameter family of sausage metrics arises for the choice $a(t)=\gamma$, where $\gamma$ is an arbitrary positive constant, and $b(t)=0$. Then, the target space metric takes the form

$$
d s^{2}=\frac{1}{\gamma}\left(d X^{2}+d Y^{2}\right)
$$

and the model describes two free scalar fields $Y$ and $X$ taking values in the spaces $R$ and $U(1)$, respectively. The resulting theory, which is denoted by $R U(1)_{\gamma}$, defines a twodimensional quantum conformal field theory with vanishing beta function. The radius of the compact space $U(1)_{\gamma}$ is $1 / \sqrt{\gamma}$ and assumes all possible values as $\gamma$ ranges from 0 to $+\infty$. For $\gamma=0$, the coordinate $X$ decompactifies and the geometry describes a plane, which can also be thought at the infinite radius limit of a round sphere. For $\gamma=+\infty$, the coordinate $X$ shrinks to zero size and the geometry degenerates to a one-dimensional line. Finally, for all intermediate values of $\gamma$, we obtain the geometry of a two-dimensional cylinder. Thus, it follows that the one-parameter family of sausage metrics encompasses both conformal as well as non-conformal backgrounds.

One expects that for generic choices of the functions $a(t)$ and $b(t)$ the resulting twodimensional quantum field theory will violate scale invariance and, hence, have nonvanishing beta function. It turns out that the renormalization group flows, which result by changing the world-sheet length scale of the model, act within the above one-parameter class of metrics in a consistent way and provide a set of first order non-linear differential equations for the two yet unknown functions. They read as follows,

$$
a^{\prime}(t)=2 b^{2}(t), \quad b^{\prime}(t)=2 a(t) b(t)
$$

and they can be easily integrated by noting that the quantity

$$
\gamma^{2}=a^{2}(t)-b^{2}(t)
$$

is conserved along the flow. Hence, choosing $\gamma$ to be any arbitrary real constant, so that $a(t) \geq-b(t)$ in all generality, we obtain the solution [35]

$$
a(t)=\gamma \operatorname{coth} 2 \gamma\left(t_{0}-t\right), \quad b(t)=\frac{\gamma}{\sinh 2 \gamma\left(t_{0}-t\right)} .
$$

This solution does not depend on the sign of the integration constant $\gamma$, and so we may choose $\gamma \geq 0$ for all practical purposes. Moreover, the restriction $b(t) \geq 0$ implies that $t \leq t_{0}$, in which case the model exhibits a well defined ultra-violet limit for $t \rightarrow-\infty$. This behavior is natural for all compact spaces with positive curvature and, therefore, the physically relevant region of the sausage model is restricted to the wedge $a(t) \geq b(t) \geq 0$ of the parameter space.

There is another arbitrary integration constant $t_{0}$ appearing in the general solution, where both $a(t)$ and $b(t)$ become infinite. In fact, we have the following leading behavior near $t_{0}$,

$$
a(t) \simeq b(t) \simeq \frac{1}{2\left(t_{0}-t\right)}, \quad e^{\Phi(Y ; t)} \simeq 2 \frac{t_{0}-t}{\cosh ^{2} Y}
$$


which is independent of the parameter $\gamma$, and hence universal. It means that all renormalization group trajectories in the two-dimensional parameter space $(a, b)$ approach the diagonal line $a(t)=b(t)$ as $t \rightarrow t_{0}$, thus converging to the point $(\infty, \infty)$. We also note, by comparing the resulting metric with the $O(3)$ sigma model, that all solutions tend to the geometry of a round sphere, but with vanishing volume as $t \rightarrow t_{0}^{-}$. Then, $t_{0}$ provides a limiting upper bound in the allowed range of the renormalization group time $t$, and it makes no sense to continue the flow beyond $t=t_{0}$. The curvature also becomes infinite at the degeneration point $t=t_{0}$, thus invalidating the lowest order approximation to the beta function equations that was obtained by disregarding all higher order curvature terms. On the other hand, $t$ can vary without restriction all the way to $-\infty$, in which case the parameters reach the fixed values

$$
a(t)=\gamma, \quad b(t)=0, \quad \text { for } t=-\infty .
$$

These constant values define the line of ultra-violet points of the trajectories for all $\gamma \geq 0$, and they correspond to the quantum conformal field theories $R U(1)_{\gamma}$.

The renormalization group trajectories are hyperbola in the $(a, b)$ parameter space, and they are described by the first integral of the flow $a^{2}(t)-b^{2}(t)=\gamma^{2}$. As such, they fill up the entire physical wedge $a(t) \geq b(t) \geq 0$ by starting from the ultra-violet fixed points $(a=\gamma, b=0)$ at $t=-\infty$, and they evolve continuously without intersecting each other towards the diagonal boundary line of the wedge, $a=b \geq 0$, which they only reach asymptotically, as $t \rightarrow t_{0}$. The diagonal boundary line is also an allowed trajectory for $\gamma=0$. The renormalization group trajectories are depicted in figure 3 below.

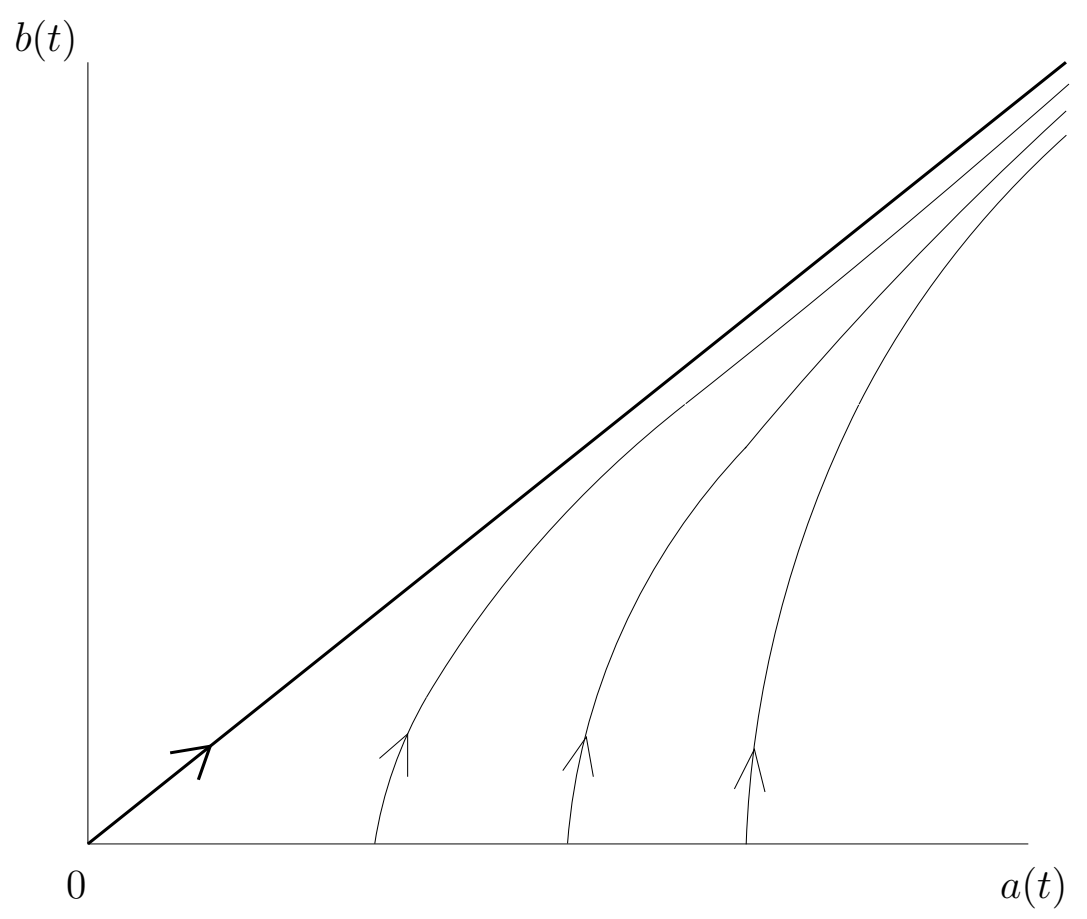

Figure 3: Renormalization group trajectories of the sausage model.

The geometry of the space changes along the trajectories in a way that is reminiscent 
of a sausage deformation of the round sphere. A way to visualize this deformation is provided by the embedding of the two-dimensional surface in three-dimensional Euclidean space, in analogy with the standard description of the $O(3)$ sigma model. For this, we introduce of an $O(3)$ unit vector field $\left\{n_{i} ; i=1,2,3\right\}$, with $\vec{n} \cdot \vec{n}=1$,

$$
\vec{n}=(\sin \theta \cos \varphi, \sin \theta \sin \varphi, \cos \theta)
$$

Then, in terms of these variables, the action $S_{\mathrm{t}}$ is cast in the form 35 .

$$
S_{\mathrm{t}}=\frac{1}{g^{2}(t)} \int \frac{(\partial \vec{n})^{2}}{1-\gamma^{2} n_{3}^{2} / g^{2}(t)} d^{2} w ; \quad \text { with } g^{2}(t)=\gamma \operatorname{coth} \gamma\left(t_{0}-t\right)
$$

and describes an axially symmetric sausage configuration embedded in three-dimensional Euclidean space spanned by $n_{1}, n_{2}$ and $n_{3}$. The sausage is elongated in the direction $n_{3}$, which acts as the axis of symmetry in three-dimensional Euclidean space, as depicted in figure 4 below.

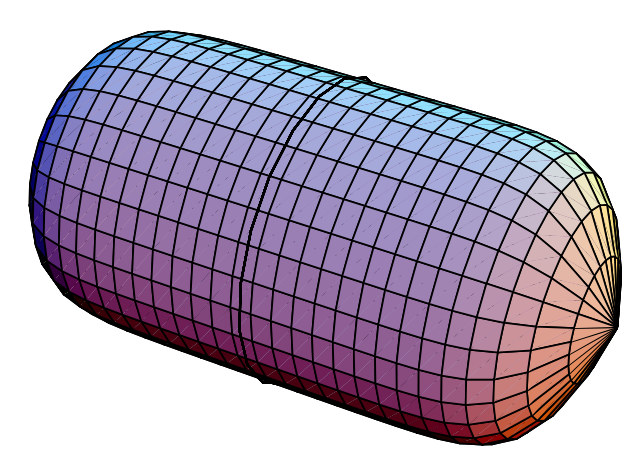

Figure 4: A sphere deforming to a sausage configuration.

The sausage becomes infinite long at the ultra-violet points, where it looks like a cylinder $R \times S^{1}$ with radius equal to $1 / \sqrt{\gamma}$. The theory is asymptotically free in the ultra-violet region, as expected on general grounds, and corresponds to the quantum conformal field theories $R U(1)_{\gamma}$ for all $\gamma$. As $t$ flows towards $t_{0}$, the configuration starts to become round with diminishing size, and it shrinks asymptotically to zero as $t \rightarrow t_{0}$, as in a big crunch. For $\gamma=0$, we have the usual spherical geometry of the $O(3)$ sigma model for all times, since the model follows the diagonal trajectory by starting from a sphere of infinite radius and evolving towards zero size; we will say more about this special solution later, in connection with the reduction of the continual Toda equation to Liouville theory.

An alternative way to visualize the geometry of the resulting configuration is described by changing coordinates to the proper variable

$$
\tilde{Y}=\sqrt{2} \cosh \gamma\left(t_{0}-t\right) \int \frac{d Y}{\sqrt{\cosh 2 Y+\cosh 2 \gamma\left(t_{0}-t\right)}}=F(\psi ; k),
$$


where $F(\psi ; k)$ denotes the incomplete elliptic integral of the first kind,

$$
F(\psi ; k)=\int_{0}^{\psi} \frac{d \chi}{\sqrt{1-k^{2} \sin ^{2} \chi}}=\int_{0}^{\sin \psi} \frac{d x}{\sqrt{\left(1-x^{2}\right)\left(1-k^{2} x^{2}\right)}},
$$

with parameters

$$
\sin \psi=\tanh Y, \quad k=\tanh \gamma\left(t_{0}-t\right) .
$$

By the defining relations of the elliptic functions, we also have $\sin \psi=\operatorname{sn}(\tilde{Y} ; k)$, where $\operatorname{sn}(u ; k)$ is the standard sine-amplitude Jacobi elliptic function with modulus $k$. The metric of the deformed configuration assumes the following form in proper coordinates $(X, \tilde{Y})$,

$$
d s_{\mathrm{t}}^{2}=\frac{k}{\gamma}\left(d \tilde{Y}^{2}+\operatorname{sn}^{2}(\tilde{Y}+K(k) ; k) d X^{2}\right),
$$

where we also made use of the identity

$$
\operatorname{sn}^{2}(u+K(k) ; k)=\frac{\mathrm{cn}^{2}(u ; k)}{\mathrm{dn}^{2}(u ; k)}=\frac{1-\mathrm{sn}^{2}(u ; k)}{1-k^{2} \operatorname{sn}^{2}(u ; k)} .
$$

Then, the shape of the space can be easily drawn, as in figure 4 above, and it represents the deformation of a sphere into an axially symmetric sausage-like configuration for all $\gamma \neq 0$, with $\tilde{Y}$ ranging from $-K(k)$ to $K(k)$.

The change of coordinates to $(X, \tilde{Y})$ has to be compensated by a vector field $\xi_{\mu}=\partial_{\mu} \tilde{\Phi}$ that describes reparametrization along the renormalization group flows. Recall that in this case one has to solve the more general system of equations

$$
\frac{\partial}{\partial t} G_{\mu \nu}=-R_{\mu \nu}+2 \nabla_{\mu} \nabla_{\nu} \tilde{\Phi}
$$

which determine the "would be" dilaton field $\tilde{\Phi}$ of the sausage configuration in proper coordinates, following the general framework of section 2.3. We find that the relevant configuration is given by

$$
\begin{aligned}
d s_{\mathrm{t}}^{2} & =\frac{1}{\gamma}\left(k d \tilde{Y}^{2}+\left(\frac{H_{1}(\tilde{Y})}{\Theta_{1}(\tilde{Y})}\right)^{2} d X^{2}\right) \\
\tilde{\Phi}(\tilde{Y}) & =\log \Theta_{1}(\tilde{Y})+\frac{1}{2}\left(\frac{E(k)}{K(k)}-\frac{1}{2} k^{\prime 2}\left(1+\frac{1}{\gamma}\right)\right) \tilde{Y}^{2},
\end{aligned}
$$

where $\Theta_{1}(u)=\Theta(u+K(k)), H_{1}(u)=H(u+K(k))$, as usual, and $K(k), E(k)$ denote the complete elliptic integrals of the first and second kind, respectively. Here, we have written the metric (4.18) and the corresponding dilaton field in terms of Jacobi theta functions, choosing the value of $\tilde{\Phi}(\tilde{Y})$ equal to $\log \Theta(K(k))$ at $\tilde{Y}=0$, rather than zero. Then, in this frame, the solution of the renormalization group flow follows immediately from the heat equation (2.24) for the theta functions. It is also useful to know in the course of the calculations that

$$
\frac{d \tau}{d k}=-\frac{i \pi}{2} \frac{1}{k k^{\prime 2} K^{2}(k)}, \quad \text { where } \tau=i \frac{K\left(k^{\prime}\right)}{K(k)} .
$$


Thus, we encounter a novel embedding of periodic solutions of the heat equation into the non-linear system of the renormalization group flows, which is applicable for all times; more examples of this embedding will be discussed in section 5.2.

The description of the sausage configuration in proper coordinates, using elliptic functions, proves advantageous for studying some special features of its geometry. The elliptic modulus is $k=\tanh \gamma\left(t_{0}-t\right)$, thus ranging from 1 to 0 as the renormalization group time $t$ flows from the ultra-violet region $t \rightarrow-\infty$ to the curvature singularity that occurs at $t=t_{0}$. The function $\operatorname{sn}(u ; k)$ is periodic in $u$, with two consecutive zeros occurring at a distance $2 K(k)$ apart from each other, and so the characteristic length of the sausage in the $\tilde{Y}$-direction is given by $2 \sqrt{k} K(k)$ for all $0 \leq k \leq 1$. For $k \simeq 1$, in particular, we have $\operatorname{cn}(u ; k) \simeq \operatorname{dn}(u ; k)$ and the geometry is an infinite long cylinder with metric $d s^{2}=\left(d \tilde{Y}^{2}+d X^{2}\right) / \gamma$, as seen from any finite distance away from the origin of the proper coordinate, $\tilde{Y}=0$. However, it is also interesting to examine the geometry of the sausage close to its tips, which are located infinite far away from $\tilde{Y}=0$ when $k=1$. It is convenient for this purpose to introduce the change of variables

$$
\rho=\tilde{Y}+K(k),
$$

by first zooming close to one of its tips, and then take the ultra-violet limit $k \rightarrow 1$. Since, all Jacobi elliptic functions become ordinary hyperbolic functions in the limit $k=1$, in particular $\operatorname{sn}(u ; 1)=\tanh u$, we obtain the following form of the metric

$$
d s_{\infty}^{2}=\frac{1}{\gamma}\left(d \rho^{2}+\tanh ^{2} \rho d X^{2}\right) .
$$

Remarkably, this is the geometry of a Euclidean two-dimensional black-hole [55, 56], which looks like an infinite long cigar, and which asymptotically becomes flat, $R \times S^{1}$, as $\rho \rightarrow+\infty^{5}$. Likewise, in the vicinity of the other tip of the sausage, $\tilde{Y} \rightarrow-K(k)$, which can be reached by first performing the change of coordinates $\rho=\tilde{Y}-K(k)$ and then taking the limit $k \rightarrow 1$, the geometry also looks like a two-dimensional black-hole, since $\operatorname{sn}^{2}(u+2 K(k) ; k)=\operatorname{sn}^{2}(u ; k) \simeq \tanh u$ for $k \simeq 1$. Thus, the infinite long cylinder that describes the conformal field theory at the ultra-violet point, $R U(1)_{\gamma}$, can be thought as being constructed by sewing together two infinite long cigars, which connect to each other in the asymptotic region at $\tilde{Y}=0$. Each of the two Euclidean black holes describes the semi-classical geometry of the $S L(2, R)_{l} / U(1)$ Wess-Zumino-Witten model, which is exact in the large level $l$ limit of the $S L(2, R)$ current algebra. In this context, we have $l=2 / \gamma$ and, therefore, the interpretation we are proposing is more appropriate when $\gamma$ is close to 0 .

\footnotetext{
${ }^{5}$ There is another interesting occurrence of the two-dimensional black-hole geometry as the infra-red fixed point of all two-dimensional cigar-like geometries, which has been studied before [57] (but see also [58]). Deforming cigars provide yet another solution of the continual Toda field equation for noncompact spaces, which, however, will not be revisited in the present work. Here, in contrast with the renormalization group flow of a single cigar, we have the occurrence of two semi-infinite cigars glued together in the ultra-violet limit of compact sigma models.
} 
The dilaton field of Witten's two-dimensional black-hole is equal to 55

$$
\tilde{\Phi}(\rho)=\log (\cosh \rho) .
$$

It is zero at $\rho=0$, and it becomes infinite at $\rho=\infty$, where the string coupling constant $\exp (-\tilde{\Phi})$ vanishes. Of course, we can also shift the dilaton by an arbitrary constant ${ }^{6}$, but this has no effect in the asymptotic region where $\tilde{\Phi}$ grows linearly, $\tilde{\Phi} \simeq \rho$, and becomes infinite as $\rho \rightarrow \infty$ (or, else, as $\tilde{Y}$ becomes finite). In our case, following equation (4.21), we find that the dilaton field is constant,

$$
\tilde{\Phi}(\tilde{Y}) \simeq \log \Theta(K(k))=\frac{1}{2} \log \left(\frac{2 K(k)}{\pi}\right),
$$

for all $\tilde{Y}$ when $k \rightarrow 1$, or when $k \rightarrow 0$. In the first case ${ }^{7}, k=1, \tilde{\Phi}$ becomes infinite everywhere by assuming the same value as in the cylindrical asymptotic region where the two black holes are glued together by matching their metric and dilaton fields. Then, the resulting "two body" problem has a dilaton which is infinite everywhere by simple superposition of the two individual "one body" problems. In the second case ${ }^{8}, k=0$, the two black holes "eat" each other and the dilaton field becomes zero, which is consistent with the creation of a curvature singularity. According to this picture, the two black holes are initially far away from each other and they start "attracting" each other by forming a sausage configuration as intermediate state that ends with a big crunch at $t=t_{0}$. Actually, it might be interesting to put this intuitive mechanical analogue on more firm basis, but we will not attempt to do so in the present work.

Summarizing, the sausage model corresponds to the following algebraic solution of the dimensionally reduced continual Toda equation in conformally flat coordinates,

$$
d s_{\mathrm{t}}^{2}=\frac{d X^{2}+d Y^{2}}{a(t)+b(t) \cosh 2 Y}=\frac{1}{\gamma} \sinh 2 \gamma\left(t_{0}-t\right) \frac{d \omega_{+} d \omega_{-}}{1+2 \omega_{+} \omega_{-} \cosh 2 \gamma\left(t_{0}-t\right)+\left(\omega_{+} \omega_{-}\right)^{2}},
$$

using the parametrization $X \in U(1), Y \in R$, or, equivalently, using a system of complex coordinates $\omega_{ \pm}$,

$$
\omega_{ \pm}=\tan \frac{\theta}{2} e^{ \pm i \varphi}=\sqrt{\frac{1-\tanh Y}{1+\tanh Y}} e^{ \pm i X} .
$$

\footnotetext{
${ }^{6}$ Adding an arbitrary constant to the dilaton changes the mass of the black hole, however, which comes out to be $M=\sqrt{\gamma} \exp (2 \tilde{\Phi}(0))$, and it represents a family of objects with variable mass inserted in a fixed background. For $\tilde{\Phi}(0)=0$, the mass is inverse to the radius of the compact coordinate $X$.

${ }^{7}$ Here, $\tilde{\Phi}(\tilde{Y})$ is equal to $\log \Theta(K(k))$ at any finite distance away from the origin $\tilde{Y}=0$, as it can be readily seen from equation (4.21) when $k \rightarrow 1$. Likewise, we observe that at the two tips located at $\tilde{Y}= \pm K(k)$, or any finite distance away from them, the dilaton field behaves as $\tilde{\Phi} \simeq \log \Theta(0)+K(k) / 2$, since $E(k) \rightarrow 1$ and $k^{\prime} K(k) \rightarrow 0$ as $k \rightarrow 1$. Moreover, since $\log \Theta(0)=\log \Theta(K(k))+\log \sqrt{k^{\prime}}$, we arrive immediately at the desired expression using the fact that $K(k)+\log k^{\prime}$ is finite $(=\log 4)$ for $k=1$.

${ }^{8}$ Here, $\tilde{\Phi}(\tilde{Y}=0)=\log \Theta(K(0))=0$, since $K(0)=\pi / 2$, and it does not make sense to have $\tilde{Y} \neq 0$; in this case, the distance shrinks to zero size, although $-\pi / 2 \leq \tilde{Y} \leq \pi / 2$, because the metric (4.21) scales with $k$ and tends to zero.
} 
For $\gamma=0$, we note separately that the target space metric has the exact form

$$
e^{\Phi\left(\omega_{+}, \omega_{-} ; t\right)}=2 \frac{\left(t_{0}-t\right)}{\left(1+\omega_{+} \omega_{-}\right)^{2}}
$$

for all $-\infty<t \leq t_{0}$, which corresponds to the geometry of a collapsing round sphere, as noted before. Actually, in this case, the radius of the sphere equals to $\sqrt{t_{0}-t}$ and so, its volume,

$$
V(t)=4 \pi\left(t_{0}-t\right),
$$

depends linearly on $t$, as expected on general grounds given in section 2.1. The collapsing round sphere is an exact solution of the continual Toda field equation, which is obtained by embedding solutions of the Liouville equation with factorized linear dependence in $t$, as in equation (3.51) (see also equation (2.2)). Thus, in the nomenclature used earlier, the diagonal line $a(t)=b(t)$ is a Liouville line in the two-dimensional parameter space of the sausage model, which also has a very simple free field realization. All other hyperbolic trajectories in the parameter space with $\gamma \neq 0$ are lying beyond this simple Liouville embedding and their free field realizations require full use of the algebraic methods of section 3.3 based on Bäcklund transformation for the continual Lie algebra $\mathcal{G}(d / d t ; 1)$.

\section{Free field realization}

We are turning now to the free field realization of the sausage configuration based on the algebraic construction of the most general solution of the continual Toda field equations. We choose to work in the conformally flat system of coordinates $(X, Y)$, and note that in the asymptotic region of $Y$, i.e., $Y \rightarrow \pm \infty$, the conformal factor of the sausage metric goes to zero as follows:

$$
e^{\Phi(Y ; t)} \simeq \frac{4}{b(t)} e^{-2|Y|}, \quad Y \rightarrow \pm \infty .
$$

Since $\Phi(Y ; t)$ becomes asymptotically a free field, i.e., $\partial_{Y}^{2} \Phi \simeq 0$, we may select the free field configuration

$$
\Phi_{0}(Y ; t)=-2 Y+\log \left(\frac{4}{\gamma} \sinh 2 \gamma\left(t_{0}-t\right)\right)
$$

and try to reconstruct the full solution of the continual Toda field equation for all $Y$ as a power series expansion of free fields based on the Lie algebra $\mathcal{G}(d / d t ; 1)$. We will actually verify that the algebraic expansion introduced in section 3.3 coincides order by order with the Taylor series expansion of $\Phi(Y ; t)$ in powers of $\exp (-2 Y)$.

First, using the exact form of the solution, we $\operatorname{expand} \exp \Phi$ in the vicinity of $Y=+\infty$,

$$
\begin{aligned}
e^{\Phi(Y ; t) \equiv} & \frac{2}{a(t)+b(t) \cosh 2 Y}=\frac{4}{b(t)} e^{-2 Y}\left(1-2 \frac{a(t)}{b(t)} e^{-2 Y}+\left(4 \frac{a^{2}(t)}{b^{2}(t)}-1\right) e^{-4 Y}\right. \\
& \left.-4 \frac{a(t)}{b(t)}\left(2 \frac{a^{2}(t)}{b^{2}(t)}-1\right) e^{-6 Y}+\mathcal{O}\left(e^{-8 Y}\right)\right)
\end{aligned}
$$


which in turn leads to the power series expansion

$$
\begin{aligned}
\Phi(Y ; t) \equiv \quad & \log \frac{2}{a(t)+b(t) \cosh 2 Y}=\Phi_{0}(Y ; t)-2 \frac{a(t)}{b(t)} e^{-2 Y}+\left(2 \frac{a^{2}(t)}{b^{2}(t)}-1\right) e^{-4 Y} \\
& -\frac{2 a(t)}{3 b(t)}\left(4 \frac{a^{2}(t)}{b^{2}(t)}-3\right) e^{-6 Y}+\mathcal{O}\left(e^{-8 Y}\right),
\end{aligned}
$$

using the identity $\log (1+x)=x-x^{2} / 2+x^{3} / 3-x^{4} / 4+\cdots$. Similarly, we may also work with the Taylor series expansion around $Y=-\infty$.

On the other hand, the class of sausage metrics fits precisely into the special class of solutions of the continual Toda field equation for $\Phi_{0}(Y ; t)=c Y+d(t)$ with $c=-2$. Thus, we may employ the expansion (3.49) in order to verify our assertion by matching terms in the two series. Straightforward calculation shows in this case that

$$
\begin{array}{ll}
\underline{\mathcal{O}}\left(e^{-2 Y}\right): \quad & \frac{1}{4} \partial_{t} e^{\Phi_{0}(Y ; t)}=-2 \cosh 2 \gamma\left(t_{0}-t\right) e^{-2 Y} \equiv-2 \frac{a(t)}{b(t)} e^{-2 Y} \\
\underline{\mathcal{O}}\left(e^{-4 Y}\right): \quad & \frac{1}{64} \partial_{t}\left(e^{\Phi_{0}(Y ; t)} \partial_{t} e^{\Phi_{0}(Y ; t)}\right)=\cosh 4 \gamma\left(t_{0}-t\right) e^{-4 Y} \equiv\left(2 \frac{a^{2}(t)}{b^{2}(t)}-1\right) e^{-4 Y} \\
\underline{\mathcal{O}}\left(e^{-6 Y}\right): \quad & \frac{1}{2,304} \partial_{t}\left(3 e^{\Phi_{0}(Y ; t)}\left(\partial_{t} e^{\Phi_{0}(Y ; t)}\right)^{2}+e^{2 \Phi_{0}(Y ; t)} \partial_{t}^{2} e^{\Phi_{0}(Y ; t)}\right)= \\
& -\frac{2}{3} \cosh 2 \gamma\left(t_{0}-t\right)\left(4 \cosh ^{2} 2 \gamma\left(t_{0}-t\right)-3\right) e^{-6 Y} \equiv \\
& -\frac{2 a(t)}{3 b(t)}\left(4 \frac{a^{2}(t)}{b^{2}(t)}-3\right) e^{-6 Y}
\end{array}
$$

and so on. Thus, the sausage metric yields a concrete example for testing the validity of the formal algebraic power series expansion of the continual Toda field configurations in term of free fields. It also helps to get a better grasp of the interplay between the geometric deformations induced by the renormalization group flow of two-dimensional metrics and the algebraic structure that underlies their systematic description as continual Toda system for $\mathcal{G}(d / d t ; 1)$.

\section{The cane and related models}

We turn next to the geometric deformations of constant negative curvature metrics under the renormalization group flows. Contrary to the case of positive curvature metrics, which exhibit asymptotic freedom by becoming free field theories in the ultraviolet region, negative curvature metrics are expected to flow towards free field theories in the infra-red region by appropriate changes of their target space geometry. We first review the classification of constant negative curvature metrics in terms of the monodromy of the free field configurations of Liouville theory, and then we investigate the geometric deformations induced by the renormalization group flow in each case separately. In all 
cases we will only consider deformations that preserve the axial symmetry of the geometric configurations, thus simplifying the dynamics, as in the sausage model. We will use again two degrees of freedom $a(t)$ and $b(t)$ to parametrize the mini-superspace models in a consistent way and derive a system of first order differential equations for their evolution. It turns out that these geometric deformations also follow hyperbolic trajectories in the parameter space, in close analogy with the renormalization group flows of the sausage model, but the physical regions differ from one model to another; likewise, the shapes of the interpolating geometries are also different. Thus, we will be able to obtain some new, yet simple, solutions of the continual Toda field equation that describe the renormalization group flow of negatively curved spaces in different patches, which look like a cane in the infra-red limit and account for the name "cane model".

\subsection{Constant negative curvature metrics}

We consider two dimensional spaces with Euclidean signature and metric

$$
d s^{2}=2 e^{\Phi\left(z_{+}, z_{-}\right)} d z_{+} d z_{-}=\frac{8}{\Lambda^{2}} \frac{\partial_{+} F^{+}\left(z_{+}\right) \partial_{-} F^{-}\left(z_{-}\right)}{\left(1-F^{+}\left(z_{+}\right) F^{-}\left(z_{-}\right)\right)^{2}} d z_{+} d z_{-}
$$

where $F^{ \pm}\left(z_{ \pm}\right)$are functions of the variable $z_{+}$and its complex conjugate $z_{-}$. Using this parametrization of the metric in terms of the general solution of the Liouville equation with coupling constant $\Lambda^{2} / 2$, the Ricci scalar curvature of the metric is equal to $-\Lambda^{2}$, i.e.,

$$
R \equiv-2 e^{-\Phi} \partial_{+} \partial_{-} \Phi=-\Lambda^{2}
$$

modulo delta-function singularities that depend on the choice of functions $F^{ \pm}\left(z_{ \pm}\right)$. As we will see shortly, there are three different type of geometries with constant negative curvature whose classification depends solely on the monodromy of the arbitrary functions $F^{+}\left(z_{+}\right)$and $F^{-}\left(z_{-}\right)$; likewise, there can be curvature singularities at $z_{+}=0=z_{-}$, which also depend on the class of the corresponding solutions.

Let us parametrize the complex coordinates $z_{ \pm}$as follows,

$$
z_{ \pm}=e^{Y \pm i X} ; \quad-\infty<Y<+\infty, \quad 0 \leq X \leq 2 \pi
$$

which describe the standard map of an infinite long cylinder onto the plane. One end of the cylinder located at $Y=-\infty$ is mapped to the origin $z_{+}=0=z_{-}$, whereas the other end with $Y=+\infty$ is mapped to infinity in the $z$-plane; this map is also familiar from the radial quantization of two-dimensional field theories. Next, we consider $X$ independent functions for the conformal factor $\Phi(Y)$, which can be either hyperbolic or parabolic or elliptic, depending on the behavior of $F^{+}\left(z_{+}\right)$under $z_{+} \rightarrow e^{2 \pi i} z_{+}$or, equivalently, under the winding $X \rightarrow X+2 \pi$. Hyperbolic solutions have monodromy of the form $F^{+}\left(z_{+}\right) \rightarrow e^{-2 \pi \zeta} F^{+}\left(z_{+}\right)$with $\zeta$ real, parabolic solutions have monodromy $F^{+}\left(z_{+}\right) \rightarrow F^{+}\left(z_{+}\right)-2 \pi$, and elliptic solutions have monodromy $F^{+}\left(z_{+}\right) \rightarrow e^{2 \pi i \zeta} F^{+}\left(z_{+}\right)$ with $\zeta$ also real. Clearly, hyperbolic solutions can be viewed as arising from elliptic 
solutions by simple analytic continuation in $\zeta$, whereas parabolic solutions are obtained in the limit $\zeta \rightarrow 0$ from the other two classes. Also, since the solutions are independent from the sign of $\zeta$, we may choose $\zeta$ to be positive definite without loss of generality.

More explicitly, we have the following three different classes of $X$-independent solutions, up to dilation (see, for instance, [59]):

(i) Hyperbolic solutions: Choosing $F^{+}\left(z_{+}\right)=z_{+}{ }^{i \zeta}$ and $F^{-}\left(z_{-}\right)=z_{-}{ }^{i \zeta}$, we have

$$
d s^{2}=\frac{2}{\Lambda^{2}} \frac{\zeta^{2}}{z_{+} z_{-} \sin ^{2}\left(\frac{\zeta}{2} \log z_{+} z_{-}\right)} d z_{+} d z_{-}=\frac{2}{\Lambda^{2}} \frac{\zeta^{2}}{\sin ^{2} \zeta Y}\left(d X^{2}+d Y^{2}\right)
$$

with conformal factor that satisfies the equation

$$
\partial_{+} \partial_{-} \Phi-\frac{\Lambda^{2}}{2} e^{\Phi}=0
$$

Clearly, this metric describes a space of constant negative curvature $-\Lambda^{2}$, which is regular everywhere without curvature singularities. The conformal factor is periodic in the variable $Y$ and, therefore, we may restrict the values of $Y$ in the fundamental domain $-\pi / \zeta \leq Y \leq 0$, rather than taking values on the entire line $-\infty<Y<+\infty$.

Passing from the conformal variable $Y$ to the proper coordinate $\tilde{Y}$, which is defined here via $\tilde{Y}=-\log (\tan (-\zeta Y / 2))$, we may write the metric in the form

$$
d s^{2}=\frac{2}{\Lambda^{2}}\left(d \tilde{Y}^{2}+\zeta^{2} \cosh ^{2} \tilde{Y} d X^{2}\right)
$$

with $\tilde{Y}$ ranging from $-\infty$ to $+\infty$ as $Y$ ranges from $-\pi / \zeta$ to 0 . It describes a twodimensional space with constant negative curvature, which corresponds to the so called plumbing fixture metric. The $X$-independent hyperbolic solution is depicted in figure 5 below.

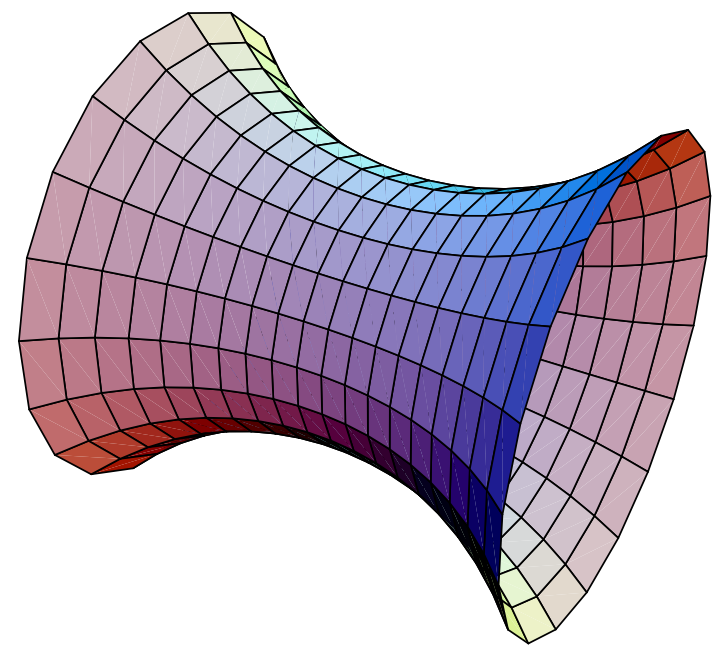

Figure 5: An axially symmetric hyperbolic solution. 
(ii) Parabolic solutions: Choosing $F^{+}\left(z_{+}\right)=i \log z_{+}$and $F^{-}\left(z_{-}\right)=i / \log z_{-}$, we have

$$
d s^{2}=\frac{8}{\Lambda^{2}} \frac{1}{z_{+} z_{-}\left(\log z_{+} z_{-}\right)^{2}} d z_{+} d z_{-}=\frac{2}{\Lambda^{2}} \frac{1}{Y^{2}}\left(d X^{2}+d Y^{2}\right),
$$

with conformal factor that satifies the equation

$$
\partial_{+} \partial_{-} \Phi-\frac{\Lambda^{2}}{2} e^{\Phi}+2 \pi \delta^{(2)}(z)=0
$$

This metric also describes a space of constant negative curvature $-\Lambda^{2}$, but in this case there is a curvature singularity at $z_{+}=0=z_{-}$, which is half the curvature of the sphere and corresponds to a puncture. In the vicinity of the singularity, however, the metric does not become flat because of the logarithmic correction. We will also restrict $Y$ in the fundamental domain $-\infty<Y \leq 0$, rather than taking values on the entire line $-\infty<Y<+\infty$.

As before, we may pass from the conformal variable $Y$ to the proper coordinate $\tilde{Y}$, which is now defined via $\tilde{Y}=-\log (-Y)$. Then, in terms of this variable the metric assumes the familiar form

$$
d s^{2}=\frac{2}{\Lambda^{2}}\left(d \tilde{Y}^{2}+e^{2 \tilde{Y}} d X^{2}\right)
$$

with $\tilde{Y}$ ranging from $-\infty$ to $+\infty$ as $Y$ ranges from $-\infty$ to 0 . The two-dimensional space exhibits rotational symmetry and can it be foliated by circles with variable size, starting from zero size at $\tilde{Y}=-\infty$ and growing exponentially to infinite size as $\tilde{Y} \rightarrow \infty$. The $X$-independent parabolic solution is depicted in figure 6 below.

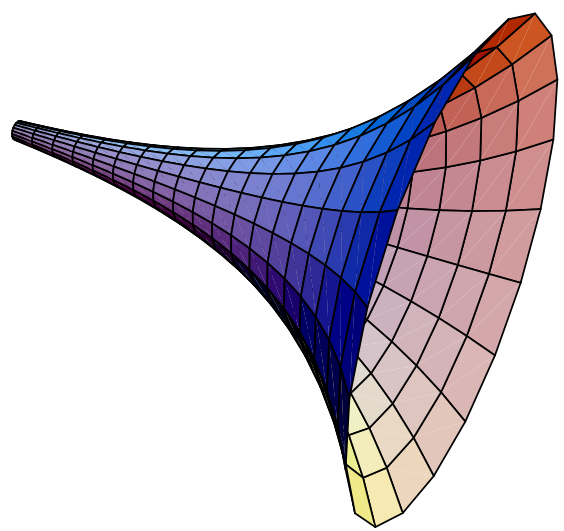

Figure 6: An axially symmetric parabolic solution.

(iii) Elliptic solutions: Choosing $F^{+}\left(z_{+}\right)=z_{+}^{\zeta}$ and $F^{-}\left(z_{-}\right)=z_{-}^{\zeta}$, with $0 \leq \zeta \leq 1$, we have the metric

$$
d s^{2}=\frac{8}{\Lambda^{2}} \frac{\zeta^{2}}{\left(z_{+} z_{-}\right)^{1-\zeta}\left(1-\left(z_{+} z_{-}\right)^{\zeta}\right)^{2}} d z_{+} d z_{-}=\frac{2}{\Lambda^{2}} \frac{\zeta^{2}}{\sinh ^{2} \zeta Y}\left(d X^{2}+d Y^{2}\right),
$$


with conformal factor that satisfies the equation

$$
\partial_{+} \partial_{-} \Phi-\frac{\Lambda^{2}}{2} e^{\Phi}+2 \pi(1-\zeta) \delta^{(2)}(z)=0
$$

As such, it describes a space of constant negative curvature $-\Lambda^{2}$, but there is also a curvature singularity at $z_{+}=0=z_{-}$, where the metric becomes flat with a conical tip and deficit angle $2 \pi(1-\zeta)$. We may also restrict the range of $Y$ in the fundamental domain $-\infty<Y \leq 0$, as before, rather than taking values on the entire real line.

It is convenient to pass from the conformal variable $Y$ to the proper coordinate $\tilde{Y}$ via $\tilde{Y}=-\log (\tanh (-\zeta Y / 2))$, in terms of which the metric assumes the form

$$
d s^{2}=\frac{2}{\Lambda^{2}}\left(d \tilde{Y}^{2}+\zeta^{2} \sinh ^{2} \tilde{Y} d X^{2}\right)
$$

with $\tilde{Y}$ ranging from 0 to $+\infty$ as $Y$ ranges from $-\infty$ to 0 . The two-dimensional space exhibits rotational symmetry and it can be foliated by circles with variable size, starting from zero size at $\tilde{Y}=0$ and expanding exponentially as $\tilde{Y} \rightarrow+\infty$. The $X$-independent elliptic solution is depicted in figure 7 below.

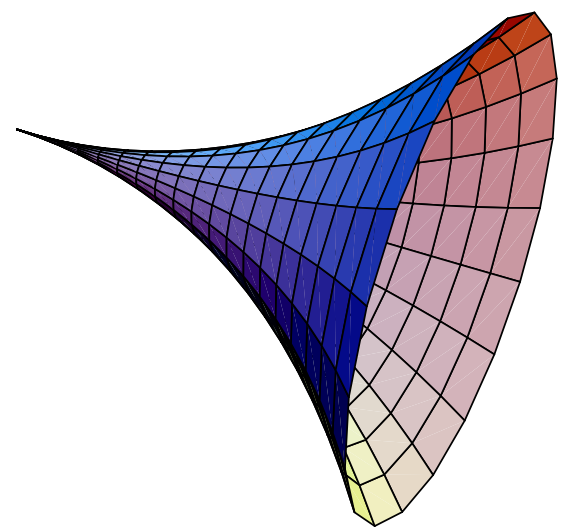

Figure 7: An axially symmetric elliptic solution.

Concluding our general discussion of the constant negative curvature metrics in two dimensions, we note that they come in three different classes depending on the monodromy of the functions $F^{ \pm}\left(z_{ \pm}\right)$. In fact, this is a simple example of a more general fact that metrics of constant negative curvature on a given manifold $\mathcal{M}$ are all locally, but not globally equivalent. Then, it is expected on general grounds that perturbative renormalization cannot reliably distinguish among such metrics and non-perturbative effects enter significantly into their renormalization [31. As a result, the geometric deformations will look different in different local patches. In two dimensions, in particular, the infra-red limit of hyperbolic and parabolic metrics looks like a cane with variable length and width, as we will see next, but the elliptic metric exhibits a very different behavior within the given set of ansatz. The presence of a conical singularity in the latter case holds the key for this differentiation in the perturbative regime. 


\subsection{Deformation of hyperbolic solutions}

We first examine the geometric deformations of the hyperbolic solution induced by the renormalization group flows. We choose to work in the conformal frame with sigma model action

$$
S_{\mathrm{t}}=\frac{1}{2} \int e^{\Phi(Y ; t)}\left(\left(\partial_{\mu} X\right)^{2}+\left(\partial_{\mu} Y\right)^{2}\right) d^{2} w
$$

Here, the conformal factor $\Phi(Y ; t)$ is given by the one-parameter family of functions

$$
e^{\Phi(Y ; t)}=\frac{2 \zeta^{2}}{a(t)+b(t) \cos (2 \zeta Y)},
$$

which preserve the axial symmetry of the configuration for all $t$, and $X$ is the angular coordinate ranging between 0 and $2 \pi$. As for the variable $Y$, it takes values in the fundamental region $-\pi / \zeta \leq Y \leq 0$, as before, but it can also be extended to the whole real axis by plumbing together the individual fundamental pieces. For $a(t)=1=-b(t)$ we have the usual hyperbolic solution with constant negative curvature $-\Lambda^{2}$, which is normalized to -1 ,

$$
e^{\Phi(Y ; t)}=\frac{\zeta^{2}}{\sin ^{2}(\zeta Y)},
$$

whereas for $b(t)=0$ we have the geometry of a flat cylinder with radius depending on the size of the parameter $a$, which has to be positive.

The metric is positive definite for all values of $t$ provided that $a(t) \geq-b(t) \geq 0$. This determines the physical wedge in the parameter space $(a(t), b(t))$ of the model, whose boundary line $a=-b$ consists by all constant negative curvature metrics with $\Lambda^{2}=a$. Using this ansatz, we find that the renormalization group flows yield the following system of first order differential equations,

$$
a^{\prime}(t)=-2 b^{2}(t), \quad b^{\prime}(t)=-2 a(t) b(t)
$$

where $a^{2}(t)-b^{2}(t)=\gamma^{2} \geq 0$ is conserved on the physical trajectories. This system is identical to the sausage model, provided that we flip the sign $t \rightarrow-t$. The sign difference is also expected on general grounds, since negative curvature spaces flow to the infra-red, $t \rightarrow+\infty$, whereas positive curvature spaces start flowing from the ultra-violet region, $t \rightarrow-\infty$. Thus, choosing $\gamma$ to be any real constant $0 \leq \gamma<+\infty$, we find the solution

$$
a(t)=\gamma \operatorname{coth} 2 \gamma\left(t-t_{0}\right), \quad b(t)=-\frac{\gamma}{\sinh 2 \gamma\left(t-t_{0}\right)},
$$

which also takes into account the appropriate restriction to the physical wedge of the parameter space ${ }^{9}$.

\footnotetext{
${ }^{9}$ If we were deforming the hyperbolic metric with $\exp \Phi=\zeta^{2} / \cos ^{2}(\zeta Y)$, which is obtained from above by a simple shift $Y \rightarrow Y+\pi /(2 \zeta)$, the sign of $b(t)$ would be opposite, thus restricting the physical wedge in the same domain as in the sausage model. In this case, the renormalization group equations, which are inert to the transformation $b(t) \rightarrow-b(t)$, lead to the same evolution as in the sausage model, but with reverse direction in the flow.
} 
The trajectories are hyperbola, as in the sausage model, but they are now filling a different region in the parameter space $(a, b)$. All trajectories start from the point $(+\infty,-\infty)$ at some arbitrary initial time $t_{0}$, where they exhibit the universal behavior

$$
a(t) \simeq-b(t) \simeq \frac{1}{2\left(t-t_{0}\right)}, \quad e^{\Phi(Y ; t)} \simeq 2\left(t-t_{0}\right) \frac{\zeta^{2}}{\sin ^{2}(\zeta Y)},
$$

which is independent of $\gamma$ for $t \rightarrow t_{0}^{+}$. Thus, the geometry starts from constant negative curvature with $\Lambda^{2}=1 / 2\left(t-t_{0}\right) \rightarrow \infty$, but there are also higher order curvature terms which can become important in this region and invalidate the lowest order approximation to the renormalization group equations. Following the renormalization group equation for $t>t_{0}$, we find that the trajectories flow towards the fixed points

$$
a=\gamma, \quad b=0
$$

as $t \rightarrow+\infty$, depending on $\gamma$. They are schematically depicted in figure 8 below.

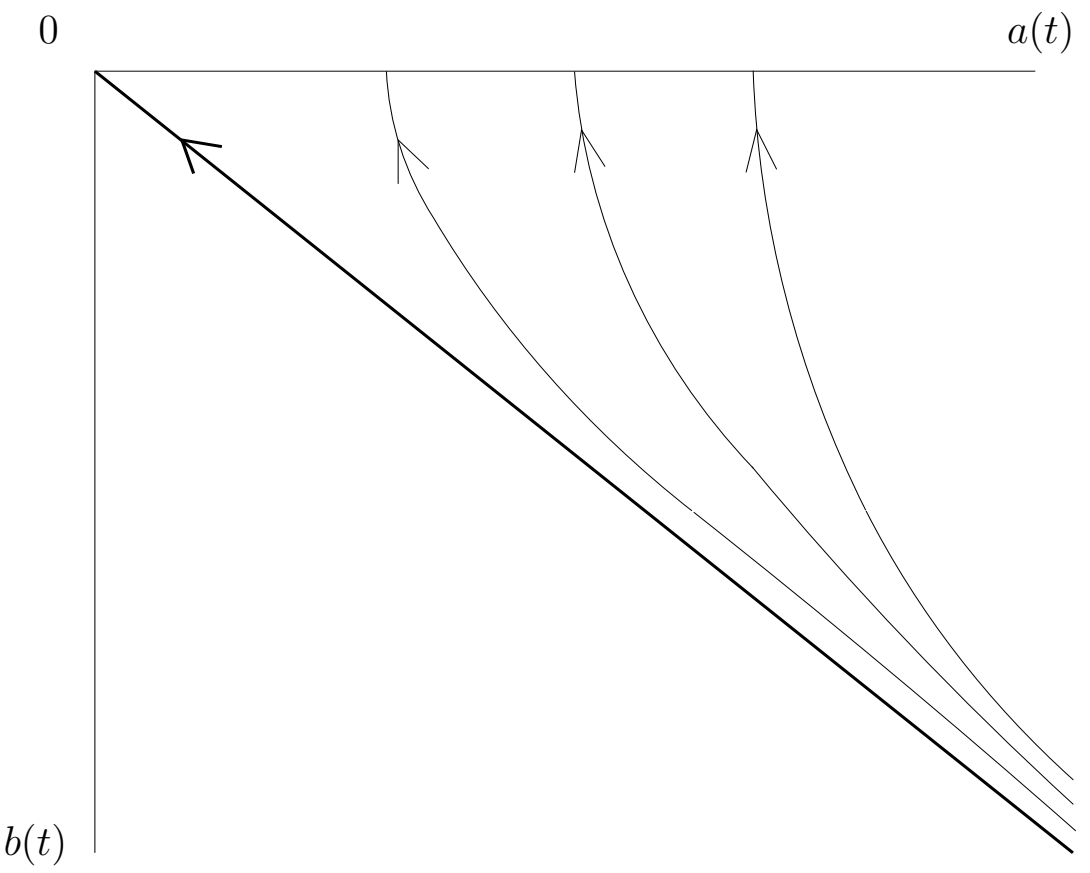

Figure 8: Renormalization group trajectories of the hyperbolic model.

The limiting points $(\gamma, 0)$ on the semi-infinite axis $b=0$ provide the set of all infra-red points of the model. In the infra-red limit the geometry is described by a flat cylinder of radius $\zeta / \sqrt{\gamma}$,

$$
d s^{2}=\frac{\zeta^{2}}{\gamma}\left(d X^{2}+d Y^{2}\right),
$$

which seemingly corresponds to the conformal field theory $R U(1)_{\gamma / \zeta^{2}}$, in close analogy with the ultra-violet fixed points of the sausage model. Actually, since the fundamental 
domain of $Y$ is taken between $-\pi / \zeta$ and 0 , the infra-red conformal field theory is only a segment of the $R U(1)_{\gamma / \zeta^{2}}$ model. It corresponds to a cylinder with finite length $\pi / \sqrt{\gamma}$ and radius $\zeta / \sqrt{\gamma}$, which describes the orbifold conformal field theory $\left(S^{1} / Z_{2}\right) \times S^{1}$, since any finite interval can be viewed as an orbifold $S^{1} / Z_{2}$ with the two edges being its fixed points. Then, in this context, the parameters $\zeta$ and $\gamma$ determine the size and the shape of the limiting infra-red geometry.

For $\gamma=0$, the radius as well as the length of the cylinder become infinite and the infra-red geometry is a two-dimensional plane, provided that $\zeta \neq 0$. For $\zeta=0$, the infra-red fixed points become identical and they correspond to degenerate cylinders with vanishing radius, provided that $\gamma \neq 0$. This behavior can also be seen in the infra-red limit of the parabolic model, since the hyperbolic conformal factor $\zeta^{2} / \sin ^{2}(\zeta Y)$ tends to the parabolic limit $1 / Y^{2}$ when $\zeta \rightarrow 0$; in that case, however, the length of the degenerate cylinder is infinite, as the fundamental region of $Y$ also becomes infinite. We will say more about the deeper relation that exists between the geometric deformations of the hyperbolic and parabolic models in the next subsection.

The boundary trajectory, $a(t)=-b(t)$, which corresponds to the value $\gamma=0$, as in the sausage model, describes the special flow

$$
e^{\Phi(Y ; t)}=2\left(t-t_{0}\right) \frac{\zeta^{2}}{\sin ^{2}(\zeta Y)}
$$

for all values of $t$. It provides the boundary Liouville line of the hyperbolic model associated to the standard embedding of Liouville theory into the renormalization group equation. The geometry of the deformation is very easy to visualize in this case, as the space remains hyperbolic for all $t_{0} \leq t<+\infty$ with constant negative curvature $-\Lambda^{2}=1 / 2\left(t_{0}-t\right)$. It starts from infinite curvature at $t=t_{0}$ and flows towards zero curvature in the infra-red limit, where it becomes flat cylinder stretched to a plane. This special flow can be intuitively understood using the membrane paradigm, where the stretching of an infinite curved hyperboloid is achieved by slowly broadening its throat to infinite size, while reducing $\Lambda^{2}$ to zero at the same time. Clearly, the final configuration is independent of $\zeta$, which is the parameter that determines the initial size of the throat. All other trajectories with $\gamma \neq 0$ describe geometric deformations of the initial hyperboloid to flat cylinders with radius $\zeta / \sqrt{\gamma}$.

We note at this point that in the vicinity of the infra-red limit the conformal factor behaves as

$$
e^{\Phi(Y ; t)} \simeq \frac{2 \zeta^{2}}{\gamma}\left(1+2 \cos (2 \zeta Y) e^{-2 \gamma\left(t-t_{0}\right)}+\mathcal{O}\left(e^{-4 \gamma\left(t-t_{0}\right)}\right)\right) \quad \text { for } t \rightarrow+\infty
$$

and describes small deviations from flatness. In this case, the metric perturbation is proportional to

$$
\Theta(Y ; t)=\cos (2 \zeta Y) \exp \left(-2 \gamma\left(t-t_{0}\right)\right)
$$

and it remains small for all $Y$. This perturbation should be compared with the analogous term $\cosh (2 Y) \exp \left(2 \gamma\left(t-t_{0}\right)\right)$ that describes the deviation of the sausage metric away 
from flatness in the vicinity of the ultra-violet points $t \rightarrow-\infty$, which, however, can become arbitrary large at the two ends of the sausage, $Y \rightarrow \pm \infty$. Then, the continual Toda equation linearizes in the infra-red region of the deformed hyperbolic model and reduces to the heat equation for $\Theta(Y ; t)$,

$$
\frac{2 \zeta^{2}}{\gamma} \frac{\partial}{\partial t} \Theta(Y ; t)=\frac{\partial}{\partial Y^{2}} \Theta(Y ; t)
$$

which is defined over a periodic spatial variable $Y$. The factor $2 \zeta^{2} / \gamma$ that appears on the left-hand side of the linearized heat equation, arises from the normalization of the metric at the infra-red points $\left.\exp \Phi\right|_{\mathrm{IR}}=2 \zeta^{2} / \gamma$. This periodic solution is a basic constituent of the Jacobi theta function that describes more general periodic solutions of the heat equation and it is smooth at $t=0$.

We are now in position to describe the geometry of the deformed hyperbolic model by changing variables from $Y$ to the proper coordinate $\tilde{Y}$,

$$
\tilde{Y}=\sqrt{2} \cosh \gamma\left(t-t_{0}\right) \int \frac{d Y}{\sqrt{\cosh 2 \gamma\left(t-t_{0}\right)-\cos 2 \zeta Y}}=-\frac{1}{\zeta} F(\psi, k) .
$$

Here, $F(\psi, k)$ is the incomplete elliptic integral of the first kind with parameter

$$
\sin \psi= \begin{cases}\cosh \gamma\left(t-t_{0}\right) \sqrt{\frac{1-\cos 2 \zeta Y}{\cosh 2 \gamma\left(t-t_{0}\right)-\cos 2 \zeta Y}} & \text { for } \quad-\frac{\pi}{2 \zeta} \leq Y \leq 0 \\ -\cos (\zeta Y) & \text { for } \quad-\frac{\pi}{\zeta} \leq Y \leq-\frac{\pi}{2 \zeta}\end{cases}
$$

and modulus $0 \leq k \leq 1$, which is equal to

$$
k=\frac{1}{\cosh \gamma\left(t-t_{0}\right)} .
$$

Then, after some calculation, the metric assumes the form

$$
d s_{\mathrm{t}}^{2}=\left\{\begin{array}{l}
\frac{\zeta^{2}}{\gamma} k^{\prime 2}\left(d \tilde{Y}^{2}+\frac{1}{k^{\prime 2}} \operatorname{dn}^{2}(\zeta \tilde{Y} ; k) d X^{2}\right) \\
\frac{\zeta^{2}}{\gamma} k^{\prime 2}\left(d \tilde{Y}^{2}+\frac{1}{\operatorname{dn}^{2}(\zeta \bar{Y} ; k)} d X^{2}\right)
\end{array}\right.
$$

respectively, where $\tilde{Y}$ ranges from $-K(k) / \zeta$ to 0 in both cases and $k^{\prime}=\sqrt{1-k^{2}}$ denotes the complementary modulus. Thanks to the identity $\operatorname{dn}(u-K(k) ; k)=k^{\prime} / \operatorname{dn}(u ; k)$, which is obeyed by the delta-amplitude Jacobi elliptic function, we arrive at the final form of the deformed metric

$$
d s_{\mathrm{t}}^{2}=\frac{\zeta^{2}}{\gamma} k^{\prime 2}\left(d \tilde{Y}^{2}+\frac{1}{k^{\prime 2}} \operatorname{dn}^{2}(\zeta \tilde{Y} ; k) d X^{2}\right)
$$

with $-2 K(k) / \zeta \leq \tilde{Y} \leq 0$ as $Y$ ranges all over the fundamental domain $-\pi / \zeta \leq Y \leq 0$.

The change of coordinates above is only valid when $\cosh 2 \gamma\left(t-t_{0}\right)$ is strictly bigger than 1 , which implies that $t>t_{0}$ or else $k<1$. This can also be seen from the resulting metric, 
which becomes ill-defined for $k^{\prime}=0$. The value $k=1$ corresponds to the initial time $t=t_{0}$, where the change of variables is simply given by $d \tilde{Y}=-\zeta d Y / \sin (\zeta Y)$ and yields the standard form of the hyperbolic metric in proper coordinates. The metric behaves well for all values $k<1$ and reaches the metric of a flat cylinder at $k=0$. The value $k=0$ corresponds to the infra-red limit of the deformations, where $\operatorname{dn}(u ; k=0)=1$ for all $u$. Also, since $\operatorname{dn}(0 ; k)=1$ for all $k$, we see immediately that the deformed geometry reduces to a very thin cylinder in the infra-red limit, when $\zeta \rightarrow 0$. We will say more about it later by studying separately the deformations of the parabolic model.

The general form of the interpolating geometry in the hyperbolic model is represented schematically in the figure 9 below, which is plotted for $k$ close to 0 and looks like a cane.

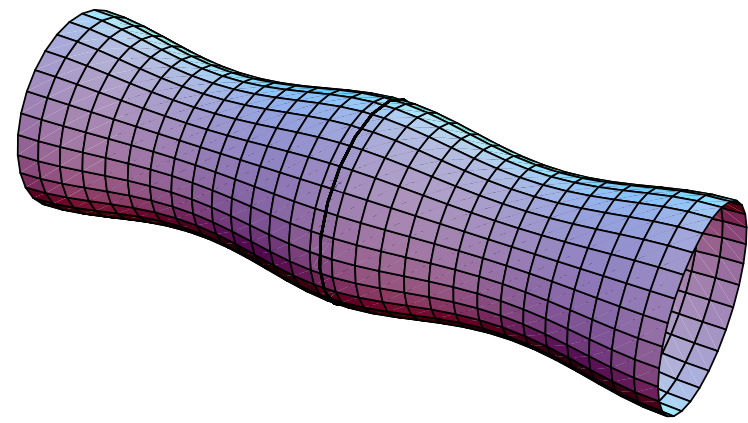

Figure 9: An axially symmetric deformed hyperbolic configuration.

The distance between the joins of the cane is equal to $2 K(k) / \zeta$, which tends to infinity when $k \rightarrow 1$ and becomes $\pi / \zeta$ when $k=0$. The mid-point in the fundamental region $-\pi / \zeta \leq Y \leq 0$, i.e., $Y=-\pi /(2 \zeta)$, corresponds to the mid-point $\tilde{Y}=-K(k) / \zeta$ in the fundamental region of the proper variable $\tilde{Y}$, where the cane makes a bump. Then, in the infra-red limit the bump disappears and we arrive at the segment of a cylinder, $\left(S^{1} / Z_{2}\right) \times S^{1}$. When $Y$ is taken on the whole real line, rather than being restricted in the fundamental domain, the deformed geometry looks like an infinite long cane, which is obtained by gluing together the individual pieces. Further details on the resulting periodic structure will also be given later in subsection 5.3.

The metric of the cane model can be expressed in terms of Jacobi theta functions, as in the sausage model, but we should also identify the "would be" dilaton field associated with the change of variables to the proper coordinates $(X, \tilde{Y})$. Using the more general system of the renormalization group flows with a vector field $\xi$ that generates the relevant reparametrizations, we find in this case that the solution is given by

$$
\begin{aligned}
d s_{\mathrm{t}}^{2} & =\frac{\zeta^{2}}{\gamma} k^{\prime}\left(k^{\prime} d \tilde{Y}^{2}+\left(\frac{\Theta_{1}(\zeta \tilde{Y})}{\Theta(\zeta \tilde{Y})}\right)^{2} d X^{2}\right), \\
\tilde{\Phi}(\tilde{Y}) & =\log \Theta(\zeta \tilde{Y})+\frac{1}{2}\left(\frac{E(k)}{K(k)}-1+\frac{1}{2} k^{2}\left(1+\frac{2}{\gamma} k^{\prime}\right)\right) \tilde{Y}^{2},
\end{aligned}
$$


with $\xi_{X}=0$ and $\xi_{\tilde{Y}}=\partial_{\tilde{Y}} \tilde{\Phi}$. One may also verify independently that this provides a solution of the renormalization group equations using the heat equation for the Jacobi theta functions for all times.

\section{Free field realization}

We complete the discussion of the hyperbolic model with the free field realization of the renormalization group trajectories. In analogy with the sausage model, we select

$$
\Phi_{0}(Y ; t)=2 i \zeta Y+\log \left(-\frac{4 \zeta^{2}}{\gamma} \sinh 2 \gamma\left(t-t_{0}\right)\right)
$$

as the corresponding free field configuration. It fits in the special class of free fields (3.47) with $c=2 i \zeta$. Then, we may verify that the algebraic power series expansion (3.49) sums up to the conformal factor $\Phi(Y ; t)$ of the deformed hyperbolic solution.

Here, we will demonstrate this for the special Liouville trajectory (5.21), which corresponds to $\gamma=0$ and has $\Phi_{0}(Y ; t)=2 i \zeta Y+\log \left(-8 \zeta^{2}\left(t-t_{0}\right)\right)$. We have, in particular, the following expansion

$$
\begin{aligned}
\Phi & =\Phi_{0}+\frac{1}{c^{2}} \partial_{t} e^{\Phi_{0}}+\frac{1}{4 c^{4}} \partial_{t}\left(e^{\Phi_{0}} \partial_{t} e^{\Phi_{0}}\right)+\frac{1}{36 c^{6}} \partial_{t}\left(3 e^{\Phi_{0}}\left(\partial_{t} e^{\Phi_{0}}\right)^{2}+e^{2 \Phi_{0}} \partial_{t}^{2} e^{\Phi_{0}}\right)+\cdots \\
& =\log \left(-8 \zeta^{2}\left(t-t_{0}\right) e^{2 i \zeta Y}\right)+2 e^{2 i \zeta Y}+e^{4 i \zeta Y}+\frac{2}{3} e^{6 i \zeta Y}+\cdots \\
& =\log \left(-8 \zeta^{2}\left(t-t_{0}\right) e^{2 i \zeta Y}\right)-2 \log \left(1-e^{2 i \zeta Y}\right)=\log \left(2\left(t-t_{0}\right) \frac{\zeta^{2}}{\sin ^{2} \zeta Y}\right)
\end{aligned}
$$

that proves our assertion. The verification of the result for $\gamma \neq 0$ is left as exercise to the interested reader.

\subsection{Deformation of parabolic solutions}

We consider the ansatz for the one-parameter family of two-dimensional metrics with

$$
e^{\Phi(Y ; t)}=\frac{1}{a(t)+b(t) Y^{2}}
$$

which describe axially symmetric deformations of the parabolic metric. For $a(t)=0$ and $b(t)=1$ the metric reduces to the standard Poincare metric on the upper (respectively lower) half-plane, which is defined for all $Y \geq 0$ (respectively $Y \leq 0$ ). For generic values of the parameters $a(t)$ and $b(t)$ the deformed geometry remains conformally flat and obeys the continual Toda field equation, provided that the following system of first order differential equations is satisfied,

$$
a^{\prime}(t)=2 a(t) b(t), \quad b^{\prime}(t)=-2 b^{2}(t) .
$$

These equations can be easily integrated by noting the existence of the conserved quantity

$$
a(t) b(t)=\gamma^{2}
$$


where $\gamma^{2}$ is taken to be a positive constant so that the metric is positive definite for all $Y$. Consequently, the general solution is given by

$$
a(t)=2 \gamma^{2}\left(t-t_{0}\right), \quad b(t)=\frac{1}{2\left(t-t_{0}\right)},
$$

where $t_{0}$ is another integration constant, which sets the lower bound in the allowed range of $\mathrm{t}, t_{0} \leq t<+\infty$, so that $a(t)$ and $b(t)$ are both positive. The final result reads as follows,

$$
e^{\Phi(Y ; t)}=\frac{2\left(t-t_{0}\right)}{4 \gamma^{2}\left(t-t_{0}\right)^{2}+Y^{2}}
$$

and provides a simple algebraic solution of the continual Toda field equation. This solution was noted before [43], but without making reference to geometry as we do.

The renormalization group trajectories are hyperbola in the $(a, b)$ parameter space using the integral of the flow, $a(t) b(t)=\gamma^{2} \geq 0$, and they fill the entire physical region with $a(t) \geq 0$ and $b(t) \geq 0$. The orbits start from the upper point $(0, \infty)$ at $t=t_{0}$ and they flow towards the lower point $(\infty, 0)$, which is a universal infra-red fixed point for all $\gamma \neq 0$. For $\gamma=0$, however, the trajectory is different and the model flows to the infra-red point $(0,0)$ located at the origin of parameter space. The physical trajectories are depicted in figure 10 below.

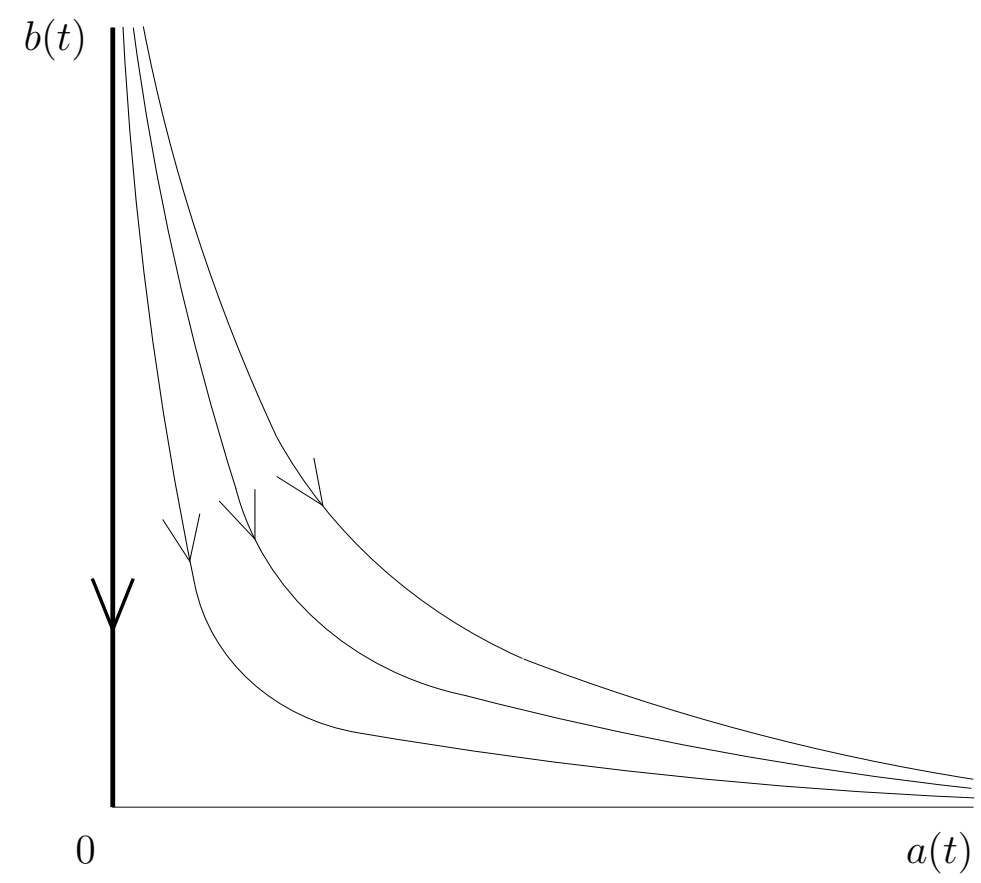

Figure 10: Renormalization group trajectories of the parabolic model.

The special trajectory with $\gamma=0$ is a Liouville line, in which case the solution reads

$$
e^{\Phi(Y ; t)}=\frac{2\left(t-t_{0}\right)}{Y^{2}}
$$


for all $t \geq t_{0}$. It corresponds to the standard embedding of Liouville theory in the continual Toda system and it is depicted by the dark line. It describes constant negative curvature spaces with $\Lambda^{2}=1 / 2\left(t-t_{0}\right)$ at all times, which start from infinity and flow to zero at the infra-red point. All other trajectories with $\gamma \neq 0$ flow towards another infra-red limit, where the geometry looks like

$$
d s_{\mathrm{t}}^{2} \simeq \frac{1}{4 \gamma^{2}\left(t-t_{0}\right)}\left(d X^{2}+d Y^{2}\right)
$$

as $t \rightarrow+\infty$. The limiting metric describes a degenerate cylinder with vanishing radius for all $\gamma \neq 0$, as it can be easily seen by rescaling the coordinates. Of course, this universal behavior is also expected following the general remarks on the infra-red limit of hyperbolic models for $\zeta \rightarrow 0$.

The shape of the interpolating geometries for the parabolic model is best described by changing variables to the proper coordinate $\tilde{Y}$,

$$
Y=2 \gamma\left(t-t_{0}\right) \sinh \left(\frac{\tilde{Y}}{\sqrt{t-t_{0}}}\right)
$$

which is appropriate for $t>t_{0}$ when $\gamma \neq 0$. Then, it is straightforward to cast the deformed metric in the form

$$
d s_{\mathrm{t}}^{2}=d \tilde{Y}^{2}+\frac{d X^{2}}{4 \gamma^{2}\left(t-t_{0}\right) \cosh ^{2}\left(\tilde{Y} / \sqrt{t-t_{0}}\right)},
$$

and the geometry looks like a blob with two spikes that extend to infinity, as depicted in figure 11 below.

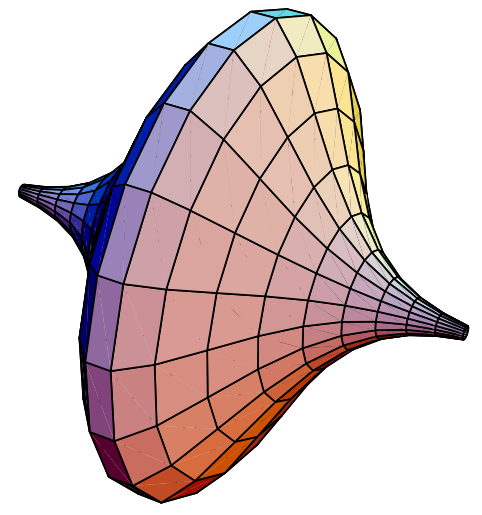

Figure 11: An axially symmetric deformed parabolic configuration.

We actually drew the picture by letting $\tilde{Y}$ range from $-\infty$ to $+\infty$ as $Y$ ranges from $-\infty$ to $+\infty$. The restriction to the fundamental domain $-\infty<Y \leq 0$ cuts the figure in half, since it also restricts the range of $\tilde{Y}$ to $-\infty<\tilde{Y} \leq 0$. It is also interesting to note that the size of the blob diminishes as $t \rightarrow+\infty$, in which case the two-dimensional 
space degenerates to a line parametrized by $\tilde{Y}$; it is nothing else but a cylinder with zero radius, which describes the infra-red limit of the model.

The deformations of the parabolic model can be fully investigated in proper coordinates $(X, \tilde{Y})$ using the generalized system of renormalization group equations that incorporate the change of coordinates along the flow. In this case, the solution is completely described by introducing the "would be" dilaton field

$$
\tilde{\Phi}(\tilde{Y})=\log \left(\cosh \frac{\tilde{Y}}{\sqrt{t-t_{0}}}\right)-\frac{\tilde{Y}^{2}}{4\left(t-t_{0}\right)}
$$

that serves to define the generating vector field of the reparametrizations, as usual, with $\xi_{X}=0$ and $\xi_{\tilde{Y}}=\partial_{\tilde{Y}} \tilde{\Phi}$. The verification is very simple and boils to the equation $R_{\tilde{Y} \tilde{Y}}=$ $2 \partial_{\tilde{Y}}^{2} \tilde{\Phi}$.

Next, we append some comments regarding the interpretation of the hyperbolic solution as an infinite long train of parabolic solutions, which are equally spaced on the axis of symmetry $\tilde{Y}$. This interpretation is useful for understanding the link between the two models and puts on firm basis the sporadic comments we made before for the behavior of the deformed geometries as $\zeta \rightarrow 0$. We will present an appropriate superposition principle that is reminiscent of free field theories, and which is also applicable to the Toda field equations by their integrability. Such superposition rules are widely used in many other integrable field theories in two dimensions; for example, static periodic solutions of the sine-Gordon model can be described as an infinite sum of equally spaced kinks and/or anti-kinks on the line (see, for instance [60, and references therein).

We already know that the conformal factor of the constant curvature hyperbolic space can be written as linear superposition of parabolic conformal factors,

$$
\frac{\zeta^{2}}{\sin ^{2}(\zeta Y)}=\sum_{n \in Z} \frac{1}{(Y-\pi n / \zeta)^{2}},
$$

which are equally spaced in $Y$. This is made possible by the integrability properties of the Liouville equation and accounts for the period of the hyperbolic solution, which is $\pi / \zeta$. Likewise, it is possible to decompose the metric (5.29) of the deformed hyperbolic model into an infinite sum of equally spaced deformed parabolic blocks, whose metric is given by equation (5.41) in proper coordinates. It is achieved by employing the remarkable identity 36

$$
\operatorname{dn}^{2}(u ; k)=\left(\frac{\pi}{2 K\left(k^{\prime}\right)}\right)^{2} \sum_{n \in Z} \frac{1}{\cosh ^{2}\left(\frac{\pi}{2 K\left(k^{\prime}\right)}(u+2 n K(k))\right)}+1-\frac{E\left(k^{\prime}\right)}{K\left(k^{\prime}\right)}
$$

that relates the two characteristic profiles for all $t>t_{0}$. This superposition is also consistent with the fact that $\operatorname{dn}(u ; k)=1 / \cosh u$ for $k=1$, in which case $K\left(k^{\prime}\right)=\pi / 2=$ $E\left(k^{\prime}\right)$ and the spacing of the lattice, $2 K(k)$, tends to infinity.

Thus, it becomes clear that as we flow towards the infra-red limit, $k \rightarrow 0$, the blobs come closer without touching each other. At the same time, the inter-connecting tubes 
are becoming thicker by superposition of the narrow spikes that join two neighboring blobs. The resulting picture looks like a cane, as shown in figure 9, and one arrives at an alternative (though more fundamental) description of the cane model in terms of its constituents. When $\zeta \rightarrow 0$ the blobs diminish in size and their separation becomes large at the same time, thus forming a very long but thin cane in the infra-red region. Thus, we obtain an alternative, yet intuitive, derivation of the infra-red limit of the deformed parabolic model, which is described by a degenerate cylinder for all $\gamma \neq 0$. The geometry of the general superposition (5.44) is depicted schematically in figure 12 below.

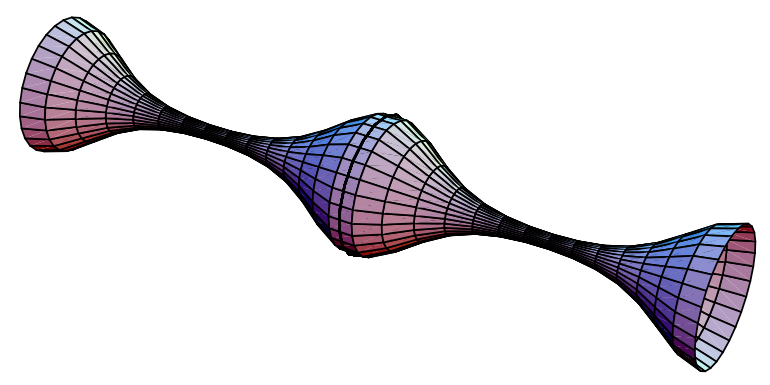

Figure 12: Superposition of deformed parabolic solutions.

\subsection{Deformation of elliptic solutions}

Let us assume that the conformal factor of the axially symmetric deformed elliptic model can be written in the form

$$
e^{\Phi(Y ; t)}=\frac{2 \zeta^{2}}{a(t)+b(t) \cosh (2 \zeta Y)},
$$

which looks like the metric of the sausage model, but it has a different domain of validity. For $a(t)=-1$ and $b(t)=1$, we obtain the standard elliptic metric with constant negative curvature and $\Lambda^{2}$ normalized to 1 . Then, we impose the restriction $b(t) \geq-a(t) \geq 0$ in order to ensure that we are describing deformations of the elliptic model; this determines the physical wedge in the parameter space $(a(t), b(t))$, which is different from the sausage model.

The first order differential equations for the evolution of the coefficients $a(t)$ and $b(t)$ are identical to the sausage model and they read as follows,

$$
a^{\prime}(t)=2 b^{2}(t), \quad b^{\prime}(t)=2 a(t) b(t)
$$

for all values of $\zeta$. The trajectories are again hyperbola in the parameter space, but in this case the conserved quantity is given by

$$
b^{2}(t)-a^{2}(t)=\gamma^{2}
$$


and the general solution is obtained by analytic continuation in time,

$$
a(t)=-\gamma \cot 2 \gamma\left(t-t_{0}\right), \quad b(t)=\frac{\gamma}{\sin 2 \gamma\left(t-t_{0}\right)}
$$

The orbits of the elliptic model with $\gamma \neq 0$ are periodic in time with period equal to $\pi / \gamma$. They all start from the same point $(-\infty,+\infty)$ in the parameter space $(a, b)$ at some initial time $t_{0}$, but after some time $t=t_{0}+\pi / 4 \gamma$ the coefficient $a(t)$ becomes zero. For later times it turns positive by approaching the Liouville line of the sausage model, as $t$ goes to $t_{0}+\pi / 2 \gamma$. This is already an indication that the model behaves badly by exiting the domain of negative curvature and entering into another domain. Continuing the validity of our solution beyond it, we find that both metric coefficients $a(t)$ and $b(t)$ turn negative by following trajectories in the lower mirror image of the upper wedge, which is clearly not physical. After a full period, the trajectories repeat themselves oscillating between the two regions of parameter space. Thus, the geometric deformations of the elliptic model do not possess an infra-red limit within the ansatz (5.45). This negative result may also be viewed as an indication that the particular mini-superspace model we are using is not physically correct when $\gamma \neq 0$. The origin of the problem we are facing here is technical and seems to be intimately related to the presence of a conical singularity, which, hence, deserves separate study. The orbits of the model are depicted schematically in figure 13 below.

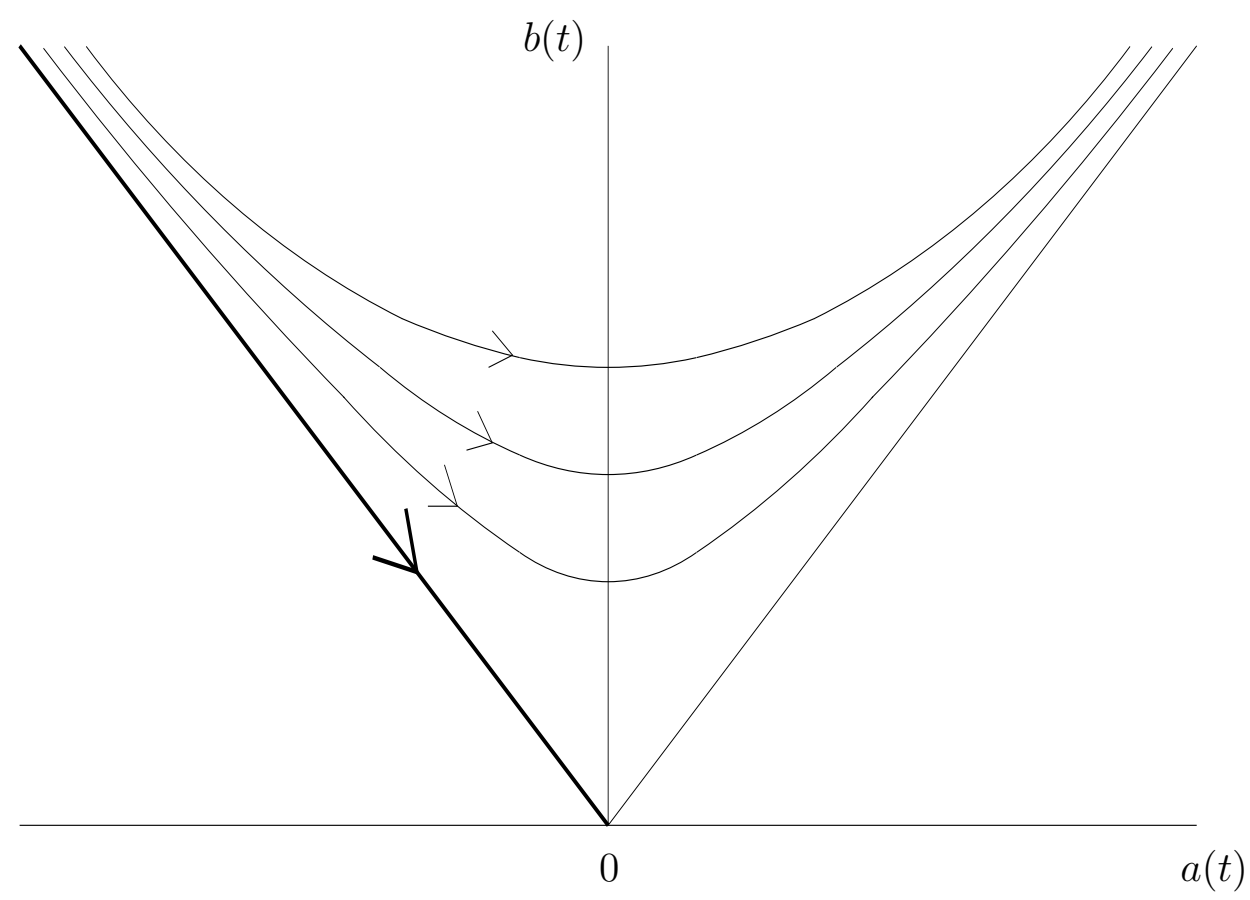

Figure 13: Renormalization group trajectories of the elliptic model.

For $\gamma=0$ we obtain the exact solution

$$
b(t)=-a(t)=\frac{1}{2\left(t-t_{0}\right)}, \quad e^{\Phi(Y ; t)}=2\left(t-t_{0}\right) \frac{\zeta^{2}}{\sinh ^{2}(\zeta Y)},
$$


which is valid for all $t>t_{0}$ up to infinity. This is the Liouville trajectory sitting at the boundary of the physical wedge, which describes a special deformation of the elliptic model via constant negative curvature metrics with $\Lambda^{2}=1 / 2\left(t-t_{0}\right)$ for all $t$. In this case, the geometry starts from infinite negative curvature and flows towards zero curvature in the infra-red region, $t \rightarrow+\infty$. Passing to proper coordinates $(X, \tilde{Y})$ with $\tilde{Y}=$ $-\log (\tanh (-\zeta Y / 2))$, as in section 5.1, and absorbing $t$ into a further redefinition of the coordinates,

$$
Y^{\prime}=2 \sqrt{t-t_{0}} \tilde{Y},
$$

we note that the metric becomes

$$
d s^{2} \simeq d Y^{\prime 2}+\zeta^{2} Y^{\prime 2} d X^{2}
$$

as $t \rightarrow+\infty$. Thus, the end-point of this special trajectory is a two-dimensional flat cone with opening angle $2 \pi \zeta$. The evolution turns a negatively curved cone into a flat cone, but the opening angle of the cone remains the same through out the process.

Summarizing, we note that there is only one physical trajectory which corresponds to the Liouville line and exhibits a well-defined infra-red behavior. This configuration can be deformed further and decay into a two-dimensional plane following another renormalization group trajectory that corresponds to different ansatz for the metric, as outlined in section 6.

\section{The decay of conical singularities}

We are turning now to other exact solutions of the renormalization group equations that describe the decay of conical singularities in non-compact spaces. These processes provide a non-linear realization of the dissipative properties of the heat equation, where it is found that the geometry exhibits a smooth limit in the infra-red region. The main example is the transition of a two-dimensional cone $C / Z_{n}$, with $n \geq 2$, to flat space, but it can also be generalized to describe the transition of $C / Z_{n}$ to another cone $C / Z_{m}$ with $m<n$. The cone is the simplest singular configuration of this kind, where the geometry is everywhere flat apart from a point. Its curvature has delta function singularity at the tip of the cone, which is similar in that regard to the initial profile of the Gaussian solution of the heat equation. The evolution under the renormalization group equation can be described in closed form, but the algebraic structure of the solution turns out to be more complicated when compared to the geometric deformations of the sausage and cane models. It can also be used as a guide to explore the behavior of the renormalization group flows in the vicinity of singular geometries in superspace. Moreover, this solution is very important for addressing the problem of tachyon condensation in closed string theory, since orbifold models (like the cone) exhibit tachyonic states in their string spectrum. These issues will be discussed next by expanding on earlier work by other authors [4, 5], using the framework of Toda field equations. Thus, tachyon condensation in closed string theory is found to exhibit a hidden relation to the infinite dimensional algebra $\mathcal{G}(d / d t ; 1)$, which 
hopefully can be used further to address many conceptual and technical questions in the future.

\subsection{Decay of a flat cone}

It is convenient to describe the geometry of a two-dimensional cone $C / Z_{n}$ using polar coordinates,

$$
d s^{2}=d r^{2}+r^{2} d \phi^{2}
$$

where $\phi$ ranges from 0 to $2 \pi / n$; the opening angle of the cone is $2 \pi / n$ and its deficit angle is $2 \pi(1-1 / n)$. This metric assumes the equivalent conformally flat form

$$
d s^{2}=2 e^{\Phi(\rho)}\left(d \rho^{2}+\rho^{2} d \theta^{2}\right)
$$

with

$$
\Phi(\rho)=2\left(\frac{1}{n}-1\right) \log \rho+\text { constant }
$$

using the change of coordinates

$$
\rho=r^{n}, \quad \theta=n \phi
$$

Here, $\theta$ runs from 0 to $2 \pi$ covering the whole two-dimensional plane instead of a single wedge with opening angle $2 \pi / n$. Using the complex coordinates $z_{ \pm}=\rho \exp ( \pm i \theta)$, the metric of a cone assumes the conformally flat form

$$
d s^{2}=\frac{2}{\left(z_{+} z_{-}\right)^{1-1 / n}} d z_{+} d z_{-}
$$

up to an overall normalization constant.

This metric is also obtained by taking the $n$-th root of the complex coordinates of the plane, which are multi-valued functions that arise naturally by moding with the $Z_{n^{-}}$ rotations $z_{ \pm} \rightarrow \exp ( \pm 2 \pi i / n) z_{ \pm}$acting on the plane. The resulting space, $C / Z_{n}$, is an orbifold whose fixed point is the tip of the cone located at $z=0$, where the curvature is infinite as $R \sim \delta(z)$. Thus, it is natural to expect that the renormalization group flow of the metric towards the infra-red region will have the tendency to smooth out the curvature singularity by diffusing it all over the place and induce a transition to the two-dimensional plane. The intuitive picture will be made explicit in the following by analyzing the exact solution that describes this very transition. Furthermore, by simple generalization, one can show that any cone with deficit angle $2 \pi(1-1 / n)$ flows towards another cone with smaller deficit angle $2 \pi(1-1 / m)$ with $m<n$.

We limit ourselves to the renormalization group flow of purely gravitational backgrounds by also allowing the freedom to perform continuous changes of the target space coordinates. Thus, we seek solutions of the lowest order equations

$$
\frac{\partial G_{\mu \nu}}{\partial t}=-R_{\mu \nu}+\nabla_{\mu} \xi_{\nu}+\nabla_{\nu} \xi_{\mu}
$$


where $\xi_{\mu}$ denote the components of the vector field that generate diffeomorphisms of the target space coordinates, and we make the ansatz [5]

$$
d s^{2}=t\left(f^{2}(r) d r^{2}+r^{2} d \phi^{2}\right), \quad \xi_{r}=\frac{1}{2} r f(r), \quad \xi_{\phi}=0 .
$$

This frame turns out to be very convenient for the description of the decay process in closed form, but later we will also transform the solution in conformally flat coordinates. Here, we are assuming a factorized linear dependence of the two-dimensional target space metric on time. The radial coordinate $r$ ranges from 0 to $\infty$, as usual, whereas the angular variable $\phi$ is taken to be periodic with arbitrary period $2 \pi / n$, thus allowing for geometries with deficit angle.

Since the components of the Ricci curvature tensor are in this frame

$$
R_{r r}=\frac{f^{\prime}(r)}{r f(r)}, \quad R_{\phi \phi}=r \frac{f^{\prime}(r)}{f^{3}(r)}, \quad R_{r \phi}=0,
$$

the renormalization group flows reduce to the following differential equation for the unknown function $f(r)$ :

$$
f^{\prime}(r)=r f^{2}(r)(1-f(r)) .
$$

It is convenient to define new variables, as

$$
x=\frac{1}{2} r^{2}, \quad y(x)=\frac{1}{f(x)}-1,
$$

since the differential equation simplifies to

$$
y^{\prime}(x)=-\frac{y(x)}{1+y(x)} .
$$

The latter is easily solved as follows,

$$
\left(\frac{1}{f(x)}-1\right) \exp \left(\frac{1}{f(x)}-1\right)=C e^{-x},
$$

where $C$ is an arbitrary positive integration constant. Then, parametrizing $C$ in terms of $n \geq 1$, as follows,

$$
C=(n-1) \exp (n-1)
$$

we observe that the function $f$ interpolates smoothly between $f=1 / n$ at $r=0$ and $f=1$ at $r=\infty,[5]$.

The solution we have described above is the most general axi-symmetric deformation of a conical space with factorized time dependence. To demonstrate our claim, let us consider the generalized ansatz

$$
\begin{aligned}
d s^{2} & =t\left(f^{2}(r) d r^{2}+g^{2}(r) d \phi^{2}\right), \\
\xi_{r} & =\xi(r), \quad \xi_{\phi}=0,
\end{aligned}
$$


which depends on three function $f(r), g(r)$ and $\xi(r)$. Explicit calculation shows in this case that the components of the Ricci curvature tensor are

$$
R_{r r}=-\frac{g^{\prime \prime}(r)}{g(r)}+\frac{f^{\prime}(r) g^{\prime}(r)}{f(r) g(r)}, \quad R_{\phi \phi}=-\frac{g(r) g^{\prime \prime}(r)}{f^{2}(r)}+\frac{g(r) g^{\prime}(r) f^{\prime}(r)}{f^{3}(r)}, \quad R_{r \phi}=0
$$

and the renormalization group equations (6.6) reduce to the following system,

$$
\begin{aligned}
& f(r)=\frac{1}{g(r)}\left(\frac{g^{\prime}(r)}{f(r)}\right)^{\prime}+2\left(\frac{\xi(r)}{f(r)}\right)^{\prime}, \\
& g(r)=\frac{1}{f(r)}\left(\frac{g^{\prime}(r)}{f(r)}\right)^{\prime}+2 \frac{g^{\prime}(r)}{f^{2}(r)} \xi(r) .
\end{aligned}
$$

Consistency of the equations implies that $\xi /(f g)$ is independent of $r$. Without loss of generality, we choose

$$
\xi(r)=\frac{1}{2} f(r) g(r)
$$

thus setting the integration constant equal to $1 / 2$. This is precisely the form of $\xi(r)$ in the ansatz (6.7) when $g(r)=r$. Then, the remaining equation simplifies to

$$
f(r) g(r)=\left(\frac{g^{\prime}(r)}{f(r)}+\frac{1}{2} g^{2}(r)\right)^{\prime}
$$

and its general solution is given by

$$
\left(\frac{g^{\prime}(r)}{f(r)}-1\right) \exp \left(\frac{g^{\prime}(r)}{f(r)}-1\right)=C \exp \left(-\frac{1}{2} g^{2}(r)\right)
$$

where $C \geq 0$ is an arbitrary integration constant. Since $g(r)$ can always be set equal to $r$ by appropriate redefinition of the radial variable, we conclude that the solution described above is indeed the most general axi-symmetric deformation of the conical space.

The explicit form of the solution (6.12), or equivalently (6.19) with $g(r)=r$ and $C$ given by equation (6.13), shows that the renormalization group flow proceeds by linear expansion of the metric, which is a global Weyl transformation. Its physical interpretation is best described by introducing a $t$-dependent change of coordinates [5], namely,

$$
\tilde{r}=r \sqrt{t}
$$

which absorbs the linear expansion scale of the metric into the radial coordinate. Then, keeping $\tilde{r}$ fixed, we see that $t \rightarrow 0$ corresponds to $r \rightarrow \infty$, and hence $f=1$, which describes a cone with opening angle $2 \pi / n$ at the initial time. On the other hand, keeping $\tilde{r}$ fixed, we see that $t \rightarrow+\infty$ corresponds to $r \rightarrow 0$, and hence $f=1 / n$, which describes the metric of a two-dimensional plane, up to an overall scale $1 / n^{2}$, at the infra-red point of the renormalization group flow. When $0 \leq t<+\infty$, the function $f(r)=f(\tilde{r} ; t)$ interpolates smoothly between the two geometries by diffusing the curvature all over the space, as it was also anticipated above on intuitive grounds. In fact, for generic values 
of $t$, the two-dimensional space is conical at infinity and smooth at the origin, as the curvature equals to

$$
R=\frac{2 f^{\prime}(r)}{r f^{3}(r)} \equiv \frac{2}{t}\left(\frac{1}{f(r)}-1\right) .
$$

The shape of this transient space is depicted schematically in figure 14 below:

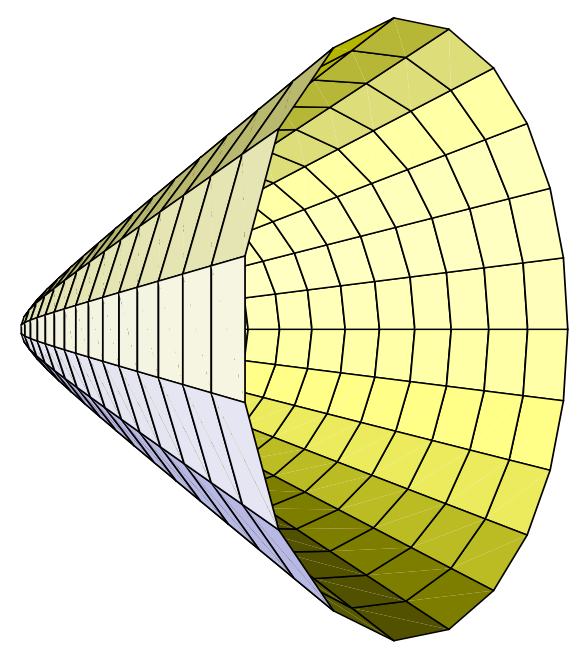

Figure 14: An asymptotically conical space with smooth tip.

The exact solution (6.12) can be used further to describe the transition of a cone $C / Z_{n}$ to another cone $C / Z_{m}$ by choosing the integration constant $C$ as

$$
C=\left(\frac{n}{m}-1\right) \exp \left(\frac{n}{m}-1\right)
$$

using two positive integers with $n>m$, [5]. In this case the angular variable $\phi$ is also taken to range between 0 and $2 \pi / n$, which equals to the opening of the cone $C / Z_{n}$ at the initial time $t=0$. Keeping $\tilde{r}$ fixed, as before, we obtain the metric of a cone with opening angle $2 \pi / m$, up to an overall scale $m^{2} / n^{2}$, as $t \rightarrow+\infty$. Successive transitions can also be considered, e.g., $n \rightarrow m \rightarrow m^{\prime} \rightarrow \cdots \rightarrow 1$, which eventually lower the value of $n$ to 1 and yield a trivial conformal field theory at the end-point of the process.

The solution that describes the transition of a cone $C / Z_{n}$ to $C / Z_{m}$ can be brought in a conformal frame. First, we consider the change of coordinates $\rho(r), \tilde{\phi}(\phi)$ given by

$$
\rho^{2}(r)=\exp \left(\frac{n}{m} \int^{r^{2} / 2} \frac{d x}{x} f(x)\right), \quad \tilde{\phi}=\frac{n}{m} \phi,
$$

which casts the metric (6.7) in the conformal form

$$
d s^{2}=2 t e^{\Phi(\rho)}\left(d \rho^{2}+\rho^{2} d \tilde{\phi}^{2}\right)
$$

with

$$
e^{\Phi(\rho)}=\frac{m^{2}}{n^{2}} \frac{r^{2}}{2 \rho^{2}} \equiv \exp \left(\int_{0}^{r^{2} / 2} \frac{d x}{x}\left(1-\frac{n}{m} f(x)\right)\right)
$$


Here, $\tilde{\phi}$ runs from 0 to $2 \pi / m$ and the metric (6.24) is conformally related to the metric of the cone $C / Z_{m}, d \rho^{2}+\rho^{2} d \tilde{\phi}^{2}$, that corresponds to the end-point of the general process $C / Z_{n} \rightarrow C / Z_{m}$. Also, the lower value of the integration range in (6.23) is left arbitrary to account for the integration constant of the transformation $\rho(r)$. Note that this change of coordinates alters the vector field $\xi$, which now becomes

$$
\xi_{\rho}=\rho \exp (\Phi / 2)
$$

according to the generalized ansatz (6.14), (6.17). The fact that $\xi_{\rho} \neq 0$ implies that $\Phi(\rho)$ above does not satisfy the Liouville equation, since, otherwise, the metric (6.24) would have been a Liouville line with factorized linear time dependence.

Next, we absorb the time dependence by the introducing the rescaling

$$
\tilde{\rho}=\sqrt{t} \rho
$$

which yields the solution of the continual Toda field equation for the transition of conical geometries in the conformal frame with $\xi_{\tilde{\rho}}=0$. The resulting Toda field $\Phi(\tilde{\rho} ; t)$ is an implicit function of $(\tilde{\rho}, t)$, which follows by inverting the transformation $\rho(r)$ in the defining relations (6.23), (6.25), 6.27) with

$$
d s^{2}=2 e^{\Phi(\tilde{\rho} ; t)}\left(d \tilde{\rho}^{2}+\tilde{\rho}^{2} d \tilde{\phi}^{2}\right) .
$$

For this reason, the solution can only be written as series expansion in the variable $\tilde{\rho}$, which, despite its limitations, will prove useful for studying the infra-red asymptotic behavior as well as the free field realization of the corresponding solution.

Before we proceed with the analysis of the general solution, note that the transformation $\rho(r)$ can be easily inverted when both $n$ and $m$ are very large, in which case the renormalization group equation linearizes and becomes the heat flow equation. Choosing $m=n-k$ for definiteness, where $k$ is arbitrary but finite, we note that $C=k / n+\mathcal{O}\left(1 / n^{2}\right)$ and $f(x)$ can be approximated by the function

$$
f(x) \simeq 1-\frac{k}{n} e^{-x}
$$

which is valid for all $x$ to lowest order in $1 / n$. Then, using equation (6.23) we obtain

$$
\rho^{2} \simeq \frac{r^{2}}{2}+\mathcal{O}\left(\frac{1}{n}\right)
$$

whereas from equation (6.25) we have

$$
\Phi(\rho) \simeq-\frac{k}{n-k} \int_{0}^{r^{2} / 2} \frac{d x}{x}\left(1-e^{-x}\right)
$$

to lowest order in $1 / n$. Thus, combining these equations, we may eliminate $r$ and write $\Phi$ as a function of $\tilde{\rho}$,

$$
\Phi(\tilde{\rho} ; t) \simeq-\frac{k}{n} \int_{0}^{\tilde{\rho}^{2} / t} \frac{d x}{x}\left(1-e^{-x}\right)+\mathcal{O}\left(\frac{1}{n^{2}}\right) .
$$


In this case, $\Phi(\tilde{\rho} ; t)$ remains small for all times, and therefore the continual Toda field equation can be approximated by the heat equation. Furthermore, using the expansion

$$
\int_{0}^{z} \frac{d x}{x}\left(1-e^{-x}\right) \simeq \mathcal{C}+\log z+\frac{e^{-z}}{z}\left(1-\frac{1}{z}+\frac{2 !}{z^{2}}-\frac{3 !}{z^{3}}+\cdots\right),
$$

which is valid for large $z=\tilde{\rho}^{2} / t$ with appropriately chosen integration constant $\mathcal{C}$, we find that the solution (6.32) interpolates smoothly between the function $\Phi(\tilde{\rho} ; t=0)=$ $-2(k / n) \log \tilde{\rho}$, which describes the conformal factor of the initial cone $C / Z_{n}$ in the reference frame of $C / Z_{n-k}$, and the constant function $\Phi(\tilde{\rho} ; t=+\infty)=0$, which corresponds to the end-point of the transition in the same reference frame. This particular expression has been derived before by considering the transition $C / Z_{n} \rightarrow C / Z_{n-2}$ with $k=2$ and $n$ large, 4].

The infra-red behavior of the geometric transition $C / Z_{n} \rightarrow C / Z_{m}$ is easily obtained when $n$ and $m$ are both finite by expanding $f(x)$ around $x=0$. The power series expansion turns out to be

$$
f(x)=\frac{m}{n}+\frac{m^{2}}{n^{2}}\left(1-\frac{m}{n}\right) x+\frac{1}{2} \frac{m^{3}}{n^{3}}\left(1-\frac{m}{n}\right)\left(2-3 \frac{m}{n}\right) x^{2}+\cdots
$$

following equation (6.12). Then, using the defining relation (6.23), we find that $\rho^{2}$ also admits the power series expansion

$$
\rho^{2}(r)=\frac{r^{2}}{2}\left(1+\frac{m}{n}\left(1-\frac{m}{n}\right) \frac{r^{2}}{2}+\frac{1}{4} \frac{m^{2}}{n^{2}}\left(1-\frac{m}{n}\right)\left(4-5 \frac{m}{n}\right) \frac{r^{4}}{4}+\cdots\right),
$$

which can be inverted to yield the following power series expansion of $r(\rho)$,

$$
\frac{r^{2}}{2 \rho^{2}}=1-\frac{m}{n}\left(1-\frac{m}{n}\right) \rho^{2}+\frac{1}{4} \frac{m^{2}}{n^{2}}\left(1-\frac{m}{n}\right)\left(4-3 \frac{m}{n}\right) \rho^{4}+\cdots .
$$

Thus, using the rescaling of the coordinates (6.27), we obtain the general infra-red behavior of the conformal factor

$$
e^{\Phi(\tilde{\rho} ; t)}=1-\frac{m}{n}\left(1-\frac{m}{n}\right) \frac{\tilde{\rho}^{2}}{t}+\mathcal{O}\left(\frac{1}{t^{2}}\right)
$$

up to an overall (but irrelevant) normalization constant $\mathrm{m}^{2} / n^{2}$.

Free field realization

Following (6.12), we may also expand $f(x)$ in an infinite series

$$
f(x)=1-C e^{-x}+\frac{1}{2 !}(2 C)^{2} e^{-2 x}-\frac{1}{3 !}(3 C)^{3} e^{-3 x}+\frac{1}{4 !}(4 C)^{4} e^{-4 x}+\cdots,
$$

which is valid for all $x$ with $C$ given by (6.22). This expansion is particularly useful when $x \rightarrow+\infty$ and only the first few terms are retained. It can be used to show that

$$
\Phi_{0}\left(z_{+}, z_{-} ; t\right)=-\left(1-\frac{m}{n}\right) \log \frac{z_{+} z_{-}}{t}, \quad \text { with } \tilde{\rho}^{2}=z_{+} z_{-}
$$

is the appropriate free field configuration that reproduces the solution of the Toda field equation given by (6.23), (6.25), (6.27), according to the perturbative series expansion following from the general formulae (3.44) and (3.45). The details of the calculation are left to the reader. 


\subsection{On the decay of a negatively curved cone}

In section 5.4 we examined deformations of the elliptic constant negative curvature metric and found that, generically, the renormalization group flow does not possess a well defined infra-red limit in the naive mini-superspace approximation. It rather exhibits an oscillatory behavior, which is not physically acceptable since the metric changes signature beyond a certain length scale, and shows that the given mini-superspace approximation is not physically correct. This behavior was attributed to the presence of a conical singularity in the class of elliptic metrics, which in proper coordinates take the form

$$
d s^{2}=d r^{2}+\zeta^{2} \sinh ^{2} r d \theta^{2} .
$$

Indeed, close to $r=0$, the metric becomes

$$
d s^{2} \simeq d r^{2}+r^{2} d \phi^{2}
$$

with $\phi=\zeta \theta$ ranging from 0 to $2 \pi \zeta$, and the space looks like a flat cone with opening angle $2 \pi \zeta$; everywhere else, away from $r=0$, it has constant negative curvature as it was discussed in section 5.1.

One may think that there are other trajectories of the renormalization group flow with well defined infra-red limit that describe the decay of a negatively curved cone (as depicted in figure 7) into a two-dimensional plane, in close analogy with the decay of a flat cone to the plane. Unfortunately, the dissipation of the singularity can not be technically achieved in this case using the axi-symmetric ansatz (6.14) with factorized linear time dependence. We have already seen that this ansatz is only applicable to the decay of a flat cone and, therefore, generalized axi-symmetric ansatz with more complicated time dependence should be devised for this purpose. So far, we have not been able to construct a solution of the renormalization group equation that describes the decay of the conical singularity in negatively curved spaces following a single trajectory in superspace. The best we can do at this moment is to view the decay of a negatively curved cone to the plane as a composite process. It proceeds by first deforming it into a flat cone using the special Liouville trajectory found in section 5.4, and then deforming the resulting cone with opening angle $2 \pi \zeta$ to the plane, along the previous lines.

It will be interesting to construct other trajectories in superspace that are capable to resolve curvature singularities in the infra-red limit, since the behavior of the renormalization group flows close to singular geometries holds the key for the systematic understanding of the dissipative properties of the continual Toda field equation. It may also help to analyze the stability properties of the flows in superspace and the physical relevance of various mini-superspace approximations.

\subsection{Tachyon condensation in closed string theory}

The renormalization group approach to the problem of tachyon condensation in closed string theory is based on the assumption that the full dynamical evolution in string theory 
can be well approximated in the world-sheet theory by flowing from one critical point to another more stable conformal solution. Instabilities in target space are indicated by the presence of tachyonic states in the string spectrum, which in turn correspond to relevant operators on the world-sheet theory. Consider ten-dimensional string backgrounds of the simple form

$$
\mathcal{C}=\mathrm{CFT}_{\mathrm{d}}+R^{9-d, 1}
$$

where $\mathrm{CFT}_{\mathrm{d}}$ is any conformal field theory block with central charge $c=d$. One may use operators $\mathcal{O}$ of the $d$-dimensional block to construct marginal operators of the full conformal background by applying momentum dressing, i.e.,

$$
\hat{\mathcal{O}}=e^{i P \cdot X} \mathcal{O} \quad \text { with } \frac{1}{2} P^{2}+\delta=1,
$$

where $(\delta, \delta)$ is the conformal dimension of $\mathcal{O}$ and $P$ is the momentum in $R^{9-d, 1}$ with coordinates $X$. Thus, the world-sheet operators $\hat{\mathcal{O}}$ correspond to space-time fluctuations with mass-squared $M^{2}=2(\delta-1)$, which are tachyonic in space-time when $\mathcal{O}$ are relevant operators on the world-sheet with $\delta<1$. Put it differently, the operator spectrum of the conformal field theory block $\mathrm{CFT}_{\mathrm{d}}$ determines the stability of the full string background under time evolution. Furthermore, the infra-red limit of the world-sheet renormalization group flow driven by $\mathcal{O}$ should coincide with the end-point of the dynamical transition from the background (6.42) to another solution of the same type, but with no tachyons in the spectrum.

It is quite difficult to provide a systematic description of this problem in all generality, but for tachyons that are localized in target space the investigation becomes more tractable. The most characteristic example, where there is an exact conformal field theory description of the spectrum, is provided by the orbifold backgrounds

$$
\mathcal{C}=C / Z_{n}+R^{7,1}
$$

where $C / Z_{n}$ is a cone with central charge $c=2$. In this case, there are tachyonic states coming from the twisted sectors of the orbifold labeled by $k=0,1,2, \cdots, n-1$. The ground state in each twisted sector is known to be tachyonic [61] (but see also [62]) with mass-squared equal to

$$
M^{2}= \begin{cases}-2 k / n, & \text { for } k \text { even } \\ -2(1-k / n), & \text { for } k \text { odd }\end{cases}
$$

which then lead to instabilities according to the general framework above. There are also excited state tachyons in many sectors, but the details are not essential for the purposes of the present work. These instabilities trigger the decay of the conformal field theory $C / Z_{n}$ to another cone $C / Z_{m}$ with $m<n$, and eventually to two-dimensional flat space $R^{2}$, which has no tachyons.

The renormalization group approach to the metric deformation that describes this transition was summarized in section 6.1 above, but recently there has also been a systematic description of the transition in real time with the same qualitative features; it 
should be mentioned, however, that the dynamical equations that describe this transition are not the same as the renormalization group equations, 24]. Furthermore, tachyon condensation in this conical space is closely related to the computation of black-hole entropy 62, which is also very interesting to consider on physical grounds.

The results we described so far show that the infinite dimensional algebra $\mathcal{G}(d / d t ; 1)$ arises as hidden symmetry of the tachyon condensation process in the regime of gravity. Different geometric transitions correspond to different solutions of the continual Toda field equation, and they should all have an infinite set of charges in target space, which are associated to the conserved currents of the system. Thus, the problem of vacuum selection in string theory appears to be part of an integrable model in superspace that consists of all target space metrics. The notion of integrability refers to the zero curvature formulation in two-dimensional target space, whereas the renormalization group time serves as a label on the root system of the underlying continual Lie algebra and it should be able to determine the form of the integrals. Unfortunately, the explicit construction of these charges, as well as their systematic interpretation in string theory remain unknown. It is natural to expect, however, that they are associated to other modes of the string which participate in the decay process.

Since tachyons become massless at the end-point of the condensation process, one is lead to consider the beta function equations of all "would be massless" fields on equal footing. We do not yet know how to extend the use of the algebra $\mathcal{G}(d / d t ; 1)$ to such generalized systems of beta function equations, which remain unexplored to a large extend, but it is certain that the inclusion of other string states will shed new light on the decay of singularities in string theory. Also, the breakdown of the lowest order approximation to the metric beta functions, which shows at some finite world-sheet length scale due to the appearance of high curvature regions in target space, is indicative of the influence of other string states to the general problem. After all, string effects always manifest as paradoxes in the lowest order effective theory of the massless modes and break down its validity. We hope to be able to report elsewhere on these interesting possibilities.

\section{Conclusions and discussion}

We have shown that the beta function equations of two-dimensional non-linear sigma models admit a zero curvature formulation with the aid of the infinite dimensional continual Lie algebra $\mathcal{G}(d / d t ; 1)$. The Cartan-Weyl generators of the algebra are sufficient to formulate the geometric deformations of two-dimensional target space metrics as an inte-

grable Toda field equation in target space using the system of conformally flat coordinates. This equation, which arises to lowest order in $\alpha^{\prime}$, provides a non-linear generalization of the heat equation and shares the same dissipative properties in renormalization group time by resolving curvature singularities in the infra-red limit of the geometries. We have constructed the general solution in terms of two-dimensional free fields, via Bäcklund transformations, and described a formal power series expansion of the non-linear field 
configurations, using group theoretic techniques as in finite dimensional Toda field theory. We have also examined several examples of axially symmetric renormalization group flows for compact and non-compact constant curvature metrics, using a mini-superspace approximation in the space of all target space metrics, and demonstrated the validity of the formal power series expansion of the general solution for appropriate choices of free field configurations.

The sausage model arises by introducing axially symmetric deformations of the standard $O(3)$ sigma model, and describes the transition of an infinite long cylinder from the ultra-violet region towards a round sphere with diminishing size at some finite world-sheet length scale. Likewise, the cane model, which was introduced here by considering axially symmetric deformations of constant negative curvature spaces, describes the transition from space with infinite negative curvature towards the infra-red region where the geometry looks like a cane with variable length and width. Both cases were also described in proper coordinates, which are more suitable for visualizing the corresponding geometric deformations, where it was found that they are parametrized by Jacobi theta functions. The latter satisfy the heat equation and, therefore, we have obtained a direct embedding of the heat equation into the non-linear system of renormalization group equations which is valid for all times. It should be noted, however, that these trajectories are physically sensible only in regions with small curvature, as all higher order curvature terms have been suppressed by considering the one-loop beta function equations. Finally, we have revisited the transition from a two-dimensional cone $C / Z_{n}$ to the plane, which is indicative of the dissipative nature of the non-linear Toda field equation to resolve conical singularities after infinitely long time. At the same time, this transition takes place between two exact conformal field theories and provides a concrete framework for studying the problem of tachyon condensation in closed string theory in the gravity regime.

The algebra $\mathcal{G}(d / d t ; 1)$ exhibits exponential growth, thus making it difficult to write the complete system of commutation relations among all generators, and, furthermore, it has no clear geometric interpretation which could be presently used for understanding the dynamics of all other string modes under the renormalization group flow. Fortunately, the complete structure of the algebra is not needed for the formulation of the metric beta functions as Toda system, but it is certainly required for making further progress in the algebraic description of the renormalization group equations. First of all, the construction of conserved currents in target space and their systematic interpretation in string theory relies on this mathematical problem. Integrability of the equations implies their existence according to the standard lore, but their construction is not straightforward as in other Toda field theories based on simple Lie algebras. The main difficulty originates from the anti-symmetric character of the Cartan kernel $\delta^{\prime}\left(t-t^{\prime}\right)$, which is also responsible for the lack of Lagrangian description of the corresponding Toda field equation. It is quite natural to expect, however, that the conserved currents in target space should be related to higher spin fields, in close analogy with the higher spin interpretation of the conserved currents in ordinary Toda field theory. As such, they should be associated to higher spin modes of string theory, but we have not succeeded so far to construct even the simplest 
representatives using local expressions in target space. There are many reasons to believe that such conservation laws will also involve non-local expressions in target space.

From a mathematical point of view, it remains to develop the structural theory of continual Lie algebras and the theory of their representations beyond the formal definition of highest weight modules that has been used in this paper. Also, the definition of bilinear forms, such as trace formulae for the Lie algebra elements of $\mathcal{G}(d / d t ; 1)$ and their products, is lacking at this moment and prevents the construction of conserved currents. Finally, it will be interesting to construct systems of roots and their reflections in a systematic way, as mirror symmetry might have a natural manifestation in this algebraic context.

The complete structure of the Lie algebra $\mathcal{G}(d / d t ; 1)$ is also important for extending the use of our methods to higher string modes beyond gravity. There has been no systematic description of the beta functions for higher string modes to this day, apart from some general results 54 that are awaiting better understanding. Also, results have been derived for some special states in the framework of two-dimensional string theory [63]. We have suggested that generalized systems of multi-component Toda field equations, which can be naturally introduced by considering the zero curvature conditions $\left[\partial_{+}+A_{+}, \partial_{-}+\right.$ $\left.A_{-}\right]=0$ with $A_{ \pm}$taking appropriate values in the subspaces $\mathcal{G}_{N} \oplus \cdots \oplus \mathcal{G}_{0} \oplus \cdots \mathcal{G}_{N}$ with $N>1$ might be relevant for the inclusion of such states in the system of renormalization group equations. If this expectation materializes, it will lead to a systematic realization of all Lie algebra elements in terms of off-shell string theory. In any case, the algebraic description of the renormalization group flows for all other additional operators, which can be added to the world-sheet action on top of the metric, pose a very interesting but difficult problem that is worth studying further.

Another important question that remains unsolved, and most likely the answer relies on the exponential growth of the Lie algebra $\mathcal{G}(d / d t ; 1)$, is the general proof of the dissipative behavior of the renormalization group flows in time. This issue is very important for the problem of tachyon condensation in closed string theory, where unstable vacua decay to more stable ones and curvature singularities dissipate away. Also, it is well known for some time that the renormalization group flows in the space of two-dimensional quantum field theories have the tendency to decrease the central charge as one moves from the ultra-violet to the infra-red fixed points. Of course, there can be some differences on the properties of Zamolodchikov's $c$-function for sigma models with compact or non-compact target spaces [20], but the essential idea is that generically the number of degrees of freedom is thinning towards the infra-red region; likewise the space-time energy decreases under the world-sheet renormalization group flow for appropriate asymptotic conditions. It will be very interesting to provide an algebraic derivation of these results based on the structure of the underlying continual Lie algebra by defining an entropy function based on its exponential growth.

The problem of tachyon condensation in closed string theory and the transitions among vacua that describe different conformal field theories is a very important issue. We believe that the algebraic methods discussed in this paper will help to understand 
the mechanism of vacuum selection in string theory, at least from the world-sheet point of view. It will be also interesting to study the meaning of our algebraic methods in real time string dynamics, as well as in other physical problems that involve time dependent gravitational backgrounds, like cosmological backgrounds and transitions that describe gravitational collapse. Finally, it will be technically useful to provide a deeper understanding of the geometric transitions based on the gauged linear sigma model approach to supersymmetric theories [64] and the powerful techniques of mirror symmetry [8, 57, 65], using the infinite dimensional algebra $\mathcal{G}(d / d t ; 1)$. All these questions are left open for future work.

\section{Note added}

After the completion of this work I became aware of several important developments in the theory of Ricci flows, which were introduced in the mathematics literature twenty years ago. Since then they have become a major tool for addressing a variety of problems in geometry in diverse dimensions; for a recent account of the main results see, for instance, [66] and references therein. Ricci flows are the same as the renormalization group flows of sigma models and, therefore, it will be interesting to compare notes in view of their physical applications to string theory.

\section{Acknowledgments}

This work was supported in part by the European Research and Training Networks "Superstring Theory" (HPRN-CT-2000-00122) and "Quantum Structure of Space-time" (HPRN-CT-2000-00131), as well as the Greek State Foundation Award "Quantum Fields and Strings" (IKYDA-2001-22) and NATO Collaborative Linkage Grant "Algebraic and Geometric Aspects of Conformal Field Theories and Superstrings" (PST.CLG.978785). A first account of the main results was presented in the annual meeting of the Hellenic society for the study of high energy physics held in the National Technical University of Athens, 17-20 April 2003; I thank the organizers for their kind invitation to participate to this enjoyable event. I also wish to thank George Savvidy for useful exchanges, as well as Brett Taylor for his help with the figures. Finally, I thank the theory division at CERN for hospitality during the final stages of this work.

\section{Dedication}

The present paper is a long due dedication to the memory of my friend Misha Saveliev

with whom I had several enlightening conversations about continual Lie algebras, Toda theories, and their physical applications. 


\section{References}

[1] A. Sen, "Non-BPS states and branes in string theory", JHEP 9806 (1998) 007.

[2] A. Sen, "Rolling tachyon", JHEP $\underline{0204}$ (2002) 048; "Tachyon matter", JHEP 0207 (2002) 065 .

[3] E. Witten, "D-branes and K-theory", JHEP $\underline{912}$ (1998) 019.

[4] A. Adams, J. Polchinski and E. Siverstein, "Don't panic! Closed string tachyons in ALE space-times", JHEP $\underline{0110}$ (2001) 029.

[5] M. Gutperle, M. Headrick, S. Minwalla and V. Schomerus, "Space-time energy decreases under world sheet RG flow", JHEP $\underline{0301}$ (2003) 073.

[6] J. Harvey, D. Kutasov and E. Martinec, "On the relevance of tachyons", hep-th/0003101, J. Harvey, D. Kutasov, E. Martinec and G. Moore, "Localized tachyons and RG flows", hep-th/0111154.

[7] E. Martinec, "Defects, decay, and dissipated states", in Progress in String, Field and Particle Theory, Cargese NATO ASI lectures, hep-th/0210231.

[8] C. Vafa, "Mirror symmetry and closed string tachyon condensation", hep-th/0111051; A. Dabholkar and C. Vafa, " $t t^{\star}$ geometry and closed string tachyon potential", JHEP $\underline{0202}(2002) 008$.

[9] J. David, M. Gutperle, M Headrick and S. Minwalla, "Closed string tachyon condensation on twisted circles", JHEP $\underline{0202}$ (2002) 041; J. David, "Unstable magnetic fluxes in heterotic string theory", JHEP $\underline{0209}$ (2002) 006.

[10] E. Martinec and G. Moore, "On decay of K-theory", hep-th/0212059.

[11] M. Saveliev, "Integro-differential non-linear equations and continual Lie algebras", Commun. Math. Phys. 121 (1989) 283.

[12] M. Saveliev and A.M. Vershik, "New examples of continuum graded Lie algebras", Phys. Lett. A143 (1990) 121.

[13] M. Saveliev and A.M. Vershik, "Continual analogs of contragredient Lie algebras (Lie algebras with a Cartan operator and nonlinear dynamical systems)", Commun. Math. Phys. 126 (1989) 367.

[14] V.G. Kac, "Infinite dimensional Lie algebras", Cambridge University Press, Cambridge, 1990.

[15] P. Goddard and D. Olive, "Algebras, lattices and strings", in Vertex Operators in Mathematics and Physics, eds. J. Lepowsky, S. Mandelstam and I.M. Singer, Publications of the Mathematical Sciences Research Institute $\underline{3}$ (1985) 51, Springer, New York. 
[16] R. Gebert and H. Nicolai, "On $E_{10}$ and the DDF construction", Commun. Math. Phys. 172 (1995) 571; "E $E_{10}$ for beginners", in Gursey Memorial Conference on Strings and Symmetries, hep-th/9411188.

[17] P. West, "Physical states and string symmetries", Mod. Phys. Lett. A10 (1995) 761; R. Gebert, H. Nicolai and P. West, "Multi-string vertices and hyperbolic Kac-Moody algebras", Int. J. Mod. Phys. A11 (1996) 429.

[18] A.B. Zamolodchikov, "Irreversibility of the flux of the renormalization group in a 2D field theory", JETP Lett. $\underline{43}$ (1986) 730.

[19] A. Tseytlin, "Conditions of Weyl invariance of the two-dimensional sigma model from equations of stationarity of the central charge action", Phys. Lett. B194 (1987) 63.

[20] J. Polchinski, "Scale and conformal invariance in quantum field theory", Nucl. Phys. $\underline{\mathrm{B} 303}$ (1988) 226.

[21] A. Tseytlin, "String vacuum backgrounds with covariantly constant null Killing vector and 2-d quantum gravity", Nucl. Phys. B390 (1993) 153.

[22] E. Kiritsis and C. Kounnas, "Dynamical topology change in string theory", Phys. Lett. B331 (1994) 51.

[23] E. Alvarez, L. Alvarez-Gaume and I. Bakas, "T-duality and space-time supersymmetry", Nucl. Phys. B457 (1995) 3.

[24] R. Gregory and J. Harvey, "Spacetime decay of cones at strong coupling", hep-th/0306146.

[25] A.B. Zamolodchikov, "Integrals of motion and S-matrix of the (scaled) $T=T_{\mathrm{c}}$ Ising model with magnetic field", Int. J. Mod. Phys. A4 (1989) 4235; "Integrable field theory from conformal field theory", Adv. Stud. Pure Math. 19 (1989) 641.

[26] A.M. Polyakov, "Interaction of Goldstone particles in two dimensions. Applications to ferromagnets and massive Yang-Mills fields", Phys. Lett. B59 (1975) 79; "Gauge Fields and Strings", Contemporary Concepts in Physics, volume 3, Harwood Academic Publishers, Chur, 1987.

[27] J. Zinn-Justin, "Quantum Field Theory and Critical Phenomena", Clarendon Press, Oxford, 1989.

[28] M.B. Green, J.H. Schwarz and E. Witten, "Superstring Theory", Cambridge University Press, Cambridge, 1988.

[29] J. Polchinski, "String Theory", Cambridge University Press, Cambridge, 1998. 
[30] G. Ecker and J. Honerkamp, "Application of invariant renormalization to the nonlinear chiral invariant pion Lagrangian in the one-loop approximation", Nucl. Phys. $\underline{\text { B35 }}$ (1971) 481; J. Honerkamp, "Chiral multi-loops", Nucl. Phys. B36 (1972) 130; J. Honerkamp, F. Krause and M. Scheunert, "On the equivalence of standard and covariant perturbation series in non-polynomial pion Lagrangian field theory", Nucl. Phys. B69 (1974) 618.

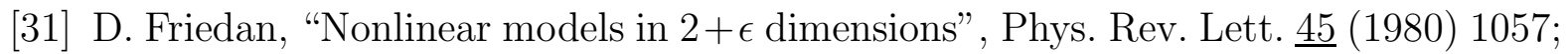
"Nonlinear models in $2+\epsilon$ dimensions", Ann. Phys. 163 (1985) 318.

[32] L. Alvarez-Gaume and D. Freedman, "Kahler geometry and the renormalization of supersymmetric sigma models", Phys. Rev. D22 (1980) 846; L. Alvarez-Gaume, D. Freedman and S. Mukhi, "The background field method and the ultraviolet structure of the supersymmetric nonlinear sigma model", Ann. Phys. 134 (1981) 85.

[33] T. Curtright and C. Zachos, "Geometry, Topology, and supersymmetry in nonlinear models", Phys. Rev. Lett. $\underline{53}$ (1984) 1799; E. Braaten, T. Curtright and C. Zachos, "Torsion and geometrostasis in nonlinear sigma models", Nucl. Phys. B260 (1985) 630 .

[34] A. Tseytlin, "Conformal anomaly in two-dimensional sigma model on curved background and strings", Phys. Lett. B178 (1986) 34; C. Callan, I. Klebanov and M. Perry, "String theory effective actions", Nucl. Phys. B278 (1986) 78; G. Shore, "A local renormalization group equation, diffeomorphisms, and conformal invariance in sigma models", Nucl. Phys. B286 (1987) 349.

[35] V.A. Fateev, E. Onofri and Al.B. Zamolodchikov, "Integrable deformations of the $O(3)$ sigma model. The sausage model", Nucl. Phys. B406 (1993) [FS] 521; L. Belardinelli, C. Destri and E. Onofri, "A numerical study of the RG equation for the deformed O(3) nonlinear sigma model", Nucl. Phys. B443 (1995) 607.

[36] E. Whittaker and G. Watson, "A Course of Modern Analysis", Cambridge University Press, Cambridge, 1927; I.S. Gradshteyn and I.M. Ryzhik, "Table of Integrals, Series, and Products", corrected and enlarged edition, Academic Press, 1980.

[37] D. Mumford, "Tata Lectures on Theta", volume I, Birkhäuser, Basel, 1983.

[38] M. Toda, "Theory of Non-linear Lattices", second edition, Springer, Berlin, 1989; M. Olshanetsky and A. Perelomov, "Classical integrable finite dimensional systems related to Lie algebras", Phys. Rept. $\underline{71}$ (1981) 313.

[39] A. Leznov and M. Saveliev, "Representation of zero curvature for the system of nonlinear partial differential equations $x_{a, z \bar{z}}=\exp (k x)_{a}$ and its integrability", Lett. Math. Phys. $\underline{3}$ (1979) 489; "Representation theory and integration of non-linear spherically symmetric equations to gauge theories", Commun. Math. Phys. $\underline{74}$ (1980) 111. 
[40] A. Leznov and M. Saveliev, "Exactly and completely integrable nonlinear dynamical systems", Acta Appl. Math. 16 (1989) 1; "Group Theoretical Methods for Integration of Non-linear Dynamical Systems", Birkhäuser, Basel, 1992.

[41] P. Mansfield, "Solution of Toda systems", Nucl. Phys. B208 (1982) 277; D. Olive and N. Turok, "Algebraic structure of Toda systems", Nucl. Phys. B220 (1983) 491.

[42] E. Floratos and J. Iliopoulos, "A note on the classical symmetries of the closed bosonic membranes", Phys. Lett. B201 (1988) 237; J. Hoppe, "Diff(A)T2 and the curvature of some infinite dimensional manifolds", Phys. Lett. B215 (1988) 706; D. Fairlie and C. Zachos, "Infinite dimensional algebras, sine brackets and $S U(\infty)$ ", Phys. Lett. B224 (1989) 101.

[43] M. Saveliev and A.M. Vershik, "A new class of infinite-dimensional Lie algebras (continuum Lie algebras) and associated nonlinear systems", in Differential Geometric Methods in Theoretical Physics, eds. C. Bartocci, U. Bruzzo and R. Cianci, SpringerVerlag, Berlin, 1990.

[44] R. Kashaev, M. Saveliev, S.A. Savelieva and A.M. Vershik, "On nonlinear equations associated with Lie algebras of diffeomorphism groups of two-dimensional manifolds", in Ideas and Methods in Mathematics and Physics, in memory of R. Hoegh-Krohn, Cambridge University Press, Cambridge, 1992.

[45] C. Boyer and J. Finley, "Killing vectors in self-dual Euclidean Einstein spaces", J. Math. Phys. 23 (1982) 1126; J. Gegenberg and A. Das, "Stationary Riemannian space-times with self-dual curvature", Gen. Rel. Grav. 16 (1984) 817.

[46] I. Bakas, "Area preserving diffeomorphisms and higher spin fields in two dimensions", in Supermembranes and Physics in $2+1$ Dimensions, eds. M. Duff, C. Pope and E. Sezgin, World Scientific, Singapore, 1990; Q-Han Park, "Extended conformal symmetries in real heavens", Phys. Lett. B236 (1990) 429.

[47] M. Saveliev, "On the integrability problem of a continuous Toda system", Theor. Math. Phys. $\underline{92}$ (1992) 1024.

[48] I. Bakas and K. Sfetsos, "Toda fields of $S O(3)$ hyper-Kähler metrics and free field realizations", Int. J. Mod. Phys. A14 (1997) 2585; "Toda fields of SO(3) hyper-Kähler metrics", Nucl. Phys. Proc. Suppl. $\underline{49}$ (1996) 10.

[49] V.G. Kac, "A sketch of Lie superalgebra theory", Commun. Math. Phys. $\underline{53}$ (1977) 31; "Lie superalgebras", Adv. Math. $\underline{26}$ (1977) 8.

[50] L. Frappat, P. Sorba and A. Sciarrino, "Dictionary of Lie Algebras and Superalgebras", Academic Press, New York, 2000.

[51] M. Saveliev and P. Sorba, "Solution of the Cauchy problem for a continuous limit of the Toda lattice and its super-extension", Lett. Math. Phys. $\underline{22}$ (1991) 119. 
[52] A. Bilal and J.-L. Gervais, "Extended $c=\infty$ conformal systems from classical Toda field theories", Nucl. Phys. B314 (1989) 646; "Systematic construction of conformal theories with higher spin Virasoro symmetries", Nucl. Phys. B318 (1989) 579.

[53] R. Gebert, T. Inami and S. Mizoguchi, "The Painleve property, W algebras and Toda field theories associated with hyperbolic Kac-Moody algebras", Int. J. Mod. Phys. A11 (1996) 5479.

[54] T. Banks and E. Martinec, "The renormalization group and string field theory", Nucl. Phys. B294 (1987) 733; Tseytlin, "Sigma model approach to string theory", Int. J. Mod. Phys. A4 (1989) 1257; "On the renormalization group approach to string equations of motion", Int. J. Mod. Phys. A4 (1989) 4249.

[55] E. Witten, "String theory and black holes", Phys. Rev. D44 (1991) 314.

[56] G. Mandal, A. Sengupta and S. Wadia, "Classical solutions of two-dimensional string theory", Mod. Phys. Lett. A6 (1991) 1685; S. Elitzur, A. Forge and E. Rabinovici, "Some global aspects of string compactifications", Nucl. Phys. B359 (1991) 581.

[57] K. Hori and A. Kapustin, "Duality of the fermionic $2 \mathrm{~d}$ black hole and $N=2$ Liouville theory as mirror symmetry", JHEP $\underline{0108}$ (2001) 045.

[58] D. Tong, "Mirror symmetry on the wall: on 2-D black holes and Liouville theory", JHEP $\underline{0304}$ (2003) 031.

[59] N. Seiberg, "Notes on quantum Liouville theory and quantum gravity", Prog. Theor. Phys. Suppl. 102 (1990) 319.

[60] I. Bakas and C. Sourdis, "Notes on periodic solitons", Fortsch. Phys. $\underline{50}$ (2002) 815.

[61] L. Dixon, J. Harvey, C. Vafa and E. Witten, "Strings on orbifolds", Nucl. Phys. B261 (1985) 678; "Strings on orbifolds 2", Nucl. Phys. B274 (1986) 285.

[62] A. Dabholkar, "Strings on a cone and black hole entropy", Nucl. Phys. B439 (1995) 650; D. Lowe and A. Strominger, "Strings near a Rindler or black hole horizon", Phys. Rev. D51 (1995) 1793; A. Dabholkar, "Tachyon condensation and black hole entropy", Phys. Rev. Lett. $\underline{88}$ (2002) 091301.

[63] E. Witten, "Ground ring of two-dimensional string theory", Nucl. Phys. B373 (1992) 187; I. Klebanov and A.M. Polyakov, "Interaction of discrete states in twodimensional string theory", Mod. Phys. Lett. A6 (1991) 3273.

[64] E. Witten, "Phases of $N=2$ theories in two dimensions", Nucl. Phys. B403 (1993) 159.

[65] K. Hori and C. Vafa, "Mirror symmetry", hep-th/0002222.

[66] H.-D. Cao and B. Chow, "Recent developments on the Ricci flow", Bull. Am. Math. Soc. $\underline{36}(1999) 59$. 